\title{
Measurement of the inclusive $b \bar{b}$ jet cross section at the Collider Detector at Fermilab
}

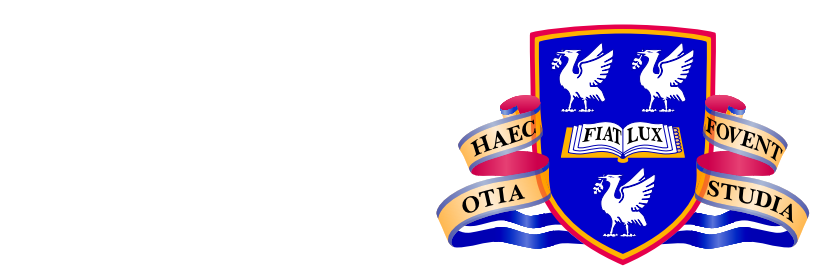

THE UNIVERSITY of LIVERPOOL

Thesis submitted in accordance with the requirements of the University of Liverpool for the degree of Doctor in Philosophy by

Anant Gajjar

September 2005 


\section{Abstract}

Data collected by the Fermilab CDF detector are used to measure the inclusive $b \bar{b}$ jet production cross section in proton-antiproton collisions at a centre-of-mass energy of $1.96 \mathrm{TeV}$. Vertices displaced from the primary interaction point (secondary vertices) are a signature for long-lived decay and are used to identify jets originating from b quarks. An event sample containing two jets, each having an identified secondary vertex, is used. The jets are required to be within the pseudo-rapidity region $|\eta|<1.2$. One of the jets is required to have a transverse energy greater than $30 \mathrm{GeV}$ and the other jet is required to have a transverse energy greater than $20 \mathrm{GeV}$. The results are compared to Leading Order (Pythia and Herwig) and Next to Leading Order (MC@NLO) predictions. 


\section{Acknowledgements}

I would like to thank my parents for all their support throughout my education. It must have been a hard decision to leave family and friends behind and settle in a foreign country for the sake of your children. Manisha, Vaishali and I will always strive to make you proud and make sure you never regret that decision. Thanks also to Mital for all her support over the past few months.

Thank you to Dave Milstead for inspiring me to stay on and do a $\mathrm{PhD}$ and Paul Booth and John Dainton for giving me the opportunity to do one. I would also like to thank Paul for all the helpful advice over the past four years. Thanks to Tara and Ronan for being such great supervisors. I really appreciate the approach you took, allowing me to get on with my work but always being there when I needed to discuss things. Thanks also to Andy Mehta who also helped and advised on the analysis and to Mike Houlden for reading through the thesis. I would also like to acknowledge the rest of the Liverpool CDF group, especially Beate and Barry, for sitting through my talks in the meetings and giving me helpful feedback. Thank you to all members of the CDF QCD group for their advice.

There are so many people in Liverpool that have been fantastic and have helped in many different ways during the past few years. I'd like to mention a few: Az, Laura Grant, Mark Tobin, Michael George, Andy Washbrook, Dave Payne, Ian Forster, Carlos, Steve Maxfield, Dave Jones, Mike Delafied, Cath Issa, Dave Hipkin, Adders, Bips, Simon Lal, Steve Mangan, Paul Reay, Si Lee, Katie Spall. There are so many more to add to this list, thanks to all of you. 
My time in Chicago was one of the most memorable moments of the past few years. I'd like to thank Martin I, D0Si, Waschke, Sinead and Thilo for helping me settle in when I moved to Chicago. Helen for putting up with me as a house mate for a year and Martin II for the other six months, and also for being such good friends. Thanks to Aidan, Tamsin, Emily and Amber for all the good times; Chris for the nights out in Wicker Park; Philip for being such a great guy and putting up with the American bashing. Thanks to Dustin for keeping me going through the winter months by coming snowboarding with me. Thanks also to all other members of the Fermi Posse, sorry I didn't mention you all, without you guys Chicago just wouldn't have been the same.

Finally, thanks to Liverpool F.C. for winning the Champions League this year. It capped off the unforgettable time I have spent in this amazing city. 


\section{Contents}

Acknowledgements

List of Tables viii

List of Figures xiv

\begin{tabular}{lll}
\hline & Introduction & 1
\end{tabular}

2 b Quark Physics in $p \bar{p}$ Collisions 3

2.1 The Standard Model . . . . . . . . . . . . . . . . . . . . 3

2.2 Electroweak Theory . . . . . . . . . . . . . . . . . . . 5

2.3 Quantum Chromodynamics . . . . . . . . . . . . . . 6

2.3 .1 Asymptotic Freedom . . . . . . . . . . . . . . 7

2.4 b Quark Production Mechanisms . . . . . . . . . . . . . . . . 9

2.5 Monte Carlo Event Generators . . . . . . . . . . . . . . . . . . 10

2.5 .1 Parton Distribution Functions (PDFs) . . . . . . . . 11

2.5 .2 Hadronisation . . . . . . . . . . . . . . . . . . 12

2.5 .3 b quark decay . . . . . . . . . . . . . . . . . . . . . 14

2.6 Previous Measurements of b Production . . . . . . . . . . . . 15

3 The Tevatron and the Collider Detector at Fermilab (CDF) 17

3.1 Accelerating the Protons and Antiprotons . . . . . . . . . 18

3.1.1 Protons . . . . . . . . . . . . . . . . . . . . . . 19

3.1 .2 Antiprotons . . . . . . . . . . . . . . . . . . . . 19

3.2 The Collider Detector at Fermilab (CDF) . . . . . . . . . . . . 20

3.2.1 Silicon Tracking System . . . . . . . . . . . . . . 22

3.2 .2 Central Outer Tracker (COT) . . . . . . . . . . . . . . 24

3.2.3 Time-of-Flight Detector (TOF) . . . . . . . . . . . . 25

3.2.4 Calorimeters . . . . . . . . . . . . . . . . . . . . . . 25

3.2 .5 Muon Systems . . . . . . . . . . . . . . . . . . . 26

3.2.6 Cherenkov Luminosity Counters (CLC) . . . . . . . . 26 
3.2 .7 Triggering . . . . . . . . . . . . . . . . . 27

3.3 Offline Event Reconstruction . . . . . . . . . . . . . . . . . . . . . 29

\begin{tabular}{lll}
\hline & Jets & 31
\end{tabular}

4.1 The Jet Clustering Algorithm . . . . . . . . . . . . . . . . . . 32

4.2 Generic Jet Energy Corrections . . . . . . . . . . . . . . . . . 34

4.2 .1 Relative Jet Energy Correction . . . . . . . . . . . . . 34

4.2 .2 Absolute Jet Energy Correction . . . . . . . . . . . . . 35

$4.2 .3 \quad$ Energy Scale Corrections . . . . . . . . . . . . . . . 35

4.2 .4 Multiple Interaction Correction . . . . . . . . . . . . 36

4.3 Correction for Jets Originating From b Quarks . . . . . . . . . . 36

4.4 Use for Cross Section Analysis . . . . . . . . . . . . . . . . . . 44

\begin{tabular}{|lll}
5 & Secondary Vertices & 45
\end{tabular}

5.1 Track Reconstruction . . . . . . . . . . . . . . . . . . . . . 46

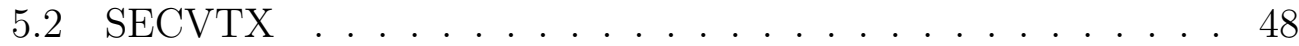

5.3 Purity of Heavy Flavour Samples . . . . . . . . . . . . . . . . 50

5.3 .1 Lepton $p_{t} \ldots \ldots \ldots \ldots \ldots \ldots$. . . . . . . . . . . 50

5.3 .2 Secondary Vertex Mass . . . . . . . . . . . . . . . 53

$5.3 .3 \quad$ Fitting the b fraction . . . . . . . . . . . . . . . . . . 53

5.4 SECVTX Efficiency Measurement . . . . . . . . . . . . . . . . 54

5.4 .1 Data Samples and Event Selection . . . . . . . . . . 55

$5.4 .2 \quad$ Efficiency Calculation . . . . . . . . . . . . . . . . . . 59

5.4 .3 Differences Between Filtered and Unfiltered Data Samples. . . . . . . . . . . . . . 64

5.4 .4 Systematic Uncertainty . . . . . . . . . . . . . . 67

5.5 Use in the Cross Section Measurement . . . . . . . . . . . . 69

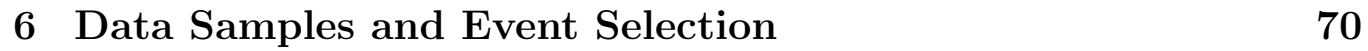

6.1 Data Samples . . . . . . . . . . . . . . . . . . 70

$6.1 .1 \quad$ Trigger Efficiency . . . . . . . . . . . . . . . . . . 71

6.2 Monte Carlo Samples . . . . . . . . . . . . . . . . . . 72

6.3 Event Selection . . . . . . . . . . . . . . . . . . 73

6.4 Acceptance . . . . . . . . . . . . . . . . . 77

$6.4 .1 \quad$ Systematic Uncertainty . . . . . . . . . . . . . . . 80

6.5 Purity of Sample . . . . . . . . . . . . . . . . . . . . . 83

6.5 .1 Systematic Uncertainties . . . . . . . . . . . . 86

$\begin{array}{llr}7 & \text { Cross Section Measurement } & 87\end{array}$

7.1 Inclusive Cross Section . . . . . . . . . . . . . . . . 87

7.1.1 Results. . . . . . . . . . . . . . . . 88 
7.2 Differential Cross Section . . . . . . . . . . . . . . . . . . . 88

$7.2 .1 \quad$ Results. . . . . . . . . . . . . . . . . . . . . . . . . . 89

7.3 Differences in Monte Carlo Cross Section Predictions . . . . . 92

7.4 Systematic errors . . . . . . . . . . . . . . . . 96

7.5 Comparison to Previous Measurements . . . . . . . . . . 97

$\begin{array}{lll}8 \text { Conclusions } & 103\end{array}$

\begin{tabular}{|ll}
\hline A Equations Defining Tower Kinematics & 105
\end{tabular}

\begin{tabular}{ll}
\hline B Checking the 60:40 Energy Ratio & 107
\end{tabular}

$\begin{array}{ll}\text { C Plots for Jet Energy Ratio using Herwig } & 110\end{array}$

\begin{tabular}{ll}
\hline D Variables used for Electron Identification & 115
\end{tabular} 


\section{List of Tables}

2.1 The fundamental particles within the Standard Model. Each fermion has an associated antiparticle which has opposite electric charge. . . . . . . . . . . . . . . . . 4

\begin{tabular}{|ll}
2.2 & Summary of previous measurements of the inclusive b quark \\
\hline & production cross section. . . . . . . . . . . . . . . . 15
\end{tabular}

$5.1 \quad$ Summary of the results of the template fits. . . . . . . . . . . . 59

$6.1 \quad$ Number of events generated and the cross section of the generated process for each of the Monte Carlo samples. . . . . . . 72

$6.2 \quad$ Change in acceptance as the jet $E_{t}$ selection cuts are increased. 79

$6.3 \quad$ Parameters for fits to the acceptance versus $E_{t}$ and dijet mass. 80

$6.4 \quad$ Systematic uncertainty on acceptance due to each of the jet energy corrections. . . . . . . . . . . . . . . . . . 82

6.5 Calculated acceptance for various PDF sets. . . . . . . . . . . 83

6.6 Results of fraction fitting to the secondary vertex mass spectrum of leading and second tagged jets in data. . . . . . . . . . 84

7.1 Inputs and results for the cross section calculation in data. Cross section predictions for Pythia, Herwig and MC@NLO are also given. All errors are statistical. . . . . . . . . . . . . . 89

$7.2 \quad$ Systematic errors on the cross section and the final value with the associated statistical and systematic error. The contributions to the total systematic error are in bold. Each contribution is split into its various components. . . . . . . . . . . . 98

7.3 Systematic errors for each bin of the differential cross section with respect to leading jet $E_{t}$ and the final values with the associated statistical and systematic error. . . . . . . . . . . . 99

7.4 Systematic errors for each bin of the differential cross section with respect to $\Delta \phi$ and the final values with the associated statistical and systematic error. . . . . . . . . . . . . . . 100 
7.5 Systematic errors for each bin of the differential cross section with respect to $M_{b \bar{b}}$ and the final values with the associated statistical and systematic error. . . . . . . . . . . . . . . . . 101 


\section{List of Figures}

2.1 Example Feynman diagrams for: a) flavour creation; b) flavour excitation; c) gluon splitting. . . . . . . . . . . . . . 9

2.2 Differential cross section predictions as a function of the azimuthal angle between the $b \bar{b}$ pair for the various categories, taken from reference [8]. . . . . . . . . . . . . . . . . . . . . 10

2.3 Schematic diagram showing how clusters $\left(C_{1}, C_{2}, \ldots\right)$ are formed using preconfinement by following the colour flow. The dashed line represents the point at which $\alpha_{s}$ becomes large. . . 14

3.1 Schematic diagram of the accelerator system. . . . . . . . . . . 18

3.2 Schematic diagram of the CDF detector. . . . . . . . . . . . . 21

3.3 The CDF coordinate system. . . . . . . . . . . . . . . 21

3.4 Arrangement of the silicon tracking system in the r-z plane. . . 22

3.5 The impact parameter resolution as a function of $p_{t}$ for the silicon system. . . . . . . . . . . . . . . . . 24

$3.6 \quad$ Functional block diagram of the data acquisition process. . . . 28

4.1 Plot showing the mean hadron (Had) jet $E_{t}$ divided by corrected calorimeter (cor Cal) jet $E_{t}$ per bin, as a function of the hadron jet $E_{t}$ (profile plot). The solid line represents a fit to a constant function, the fit parameters are shown in the top right corner. 38

4.2 Profile plots showing hadron (Had) jet $E_{t}$ divided by corrected calorimeter (cor Cal) jet $E_{t}$, as a function of the hadron jet $E_{t}$, for: a) b jets; b) c jets; c) gluon jets; d) uds jets. The solid line represents a fit to a constant function, the fit parameters are shown in the top right corner of each plot. . . . . . . . . . . 39

4.3 Profile plots showing the ratio of hadron (Had) jet $E_{t}$ to corrected calorimeter (cor Cal) jet $E_{t}$, for gluon- and uds jets combined, when the relative energy correction is adjusted by $+1 \sigma$ (left) and $-1 \sigma$ (right). The solid line represents a fit to a constant function, the fit parameters are shown in the top right corner. . . . . . . . . . . . . . . . 40 
4.4 Profile plots showing the ratio of hadron (Had) jet $E_{t}$ to corrected calorimeter (cor Cal) jet $E_{t}$, for gluon- and uds jets combined, when the jet energy scale correction is adjusted by $+1 \sigma$ (left) and $-1 \sigma$ (right). The solid line represents a fit to a constant function, the fit parameters are shown in the top right corner. . . . . . . . . . . . . . 40

4.5 Profile plots showing the ratio of hadron (Had) jet $E_{t}$ to corrected calorimeter (cor Cal) jet $E_{t}$, for gluon- and uds jets combined, when the multiple interaction correction is adjusted by $+1 \sigma$ (left) and $-1 \sigma$ (right). The solid line represents a fit to a constant function, the fit parameters are shown in the top right corner. . . . . . . . . . . . . . . 44

4.6 Profile plots showing the ratio of hadron (Had) jet $E_{t}$ to corrected calorimeter (cor Cal) jet $E_{t}$, for gluon- and uds jets combined, when the absolute energy correction is adjusted by $+1 \sigma$ (left) and $-1 \sigma$ (right). The solid line represents a fit to a constant function, the fit parameters are shown in the top right corner. . . . . . . . . . . . . . . . 44

4.7 Profile plots showing hadron ( $\mathrm{Had}$ ) jet $E_{t}$ divided by corrected calorimeter (cor Cal) jet $E_{t}$, as a function of the hadron jet $E_{t}$, for: a) non semi-leptonic $b$ decay; $b$ ) $b$ decay involving an electron; c) b decay involving a muon; d) b decay involving $a$ tau. The solid line represents a fit to a constant function, the fit parameters are shown in the top right corner. . . . . . . . . 42

4.8 Profile plot showing hadron (Had) jet $E_{t}$ divided by corrected calorimeter (cor Cal) jet $E_{t}$ for $b$ jets as a function of the hadron jet $E_{t}$ after applying the $b$ jet correction to them. The solid line represents a fit to a constant function, the fit parameters are shown in the top right corner. . . . . . . . . . . . . . . 43

5.1 Schematic diagram showing how a long-lived particle produced at the primary vertex will travel a distance $L_{x y}$ in the $r-\phi$ plane before decaying at the secondary vertex. The tracks from the decay have a large distance of closest approach $\left(d_{0}\right)$ to the primary vertex. . . . . . . . . . . . . . . . . . 46

5.2 Distributions for $p_{t}^{\text {rel }} /$ jet $E_{t}$ for electrons within $b$ jets, $c$ jets and light jets. . . . . . . . . . . . . . 51

5.3 Profile plot showing the dependence on jet $E_{t}$ of: a) $p_{t}^{\text {rel }}$; b)

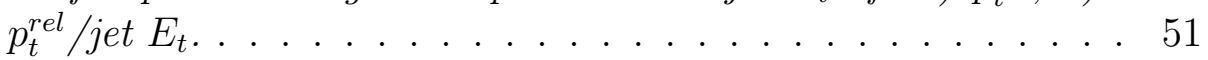

$5.4 p_{t}^{\text {rel }} /$ jet $E_{t}$ spectrum for $b$ jets for various jet energy ranges. . . 52 
5.5 Invariant mass of tracks associated with secondary vertices found within b jets, c jets and light jets. . . . . . . . . . . . . . 53

5.6 Profile plot showing the secondary vertex mass as a function of jet $E_{t}$ for $b, c$ and light jets. . . . . . . . . . . . . . 54

5.7 Comparison between Pythia and Herwig for the $p_{t}^{\text {rel }}$ templates for: a) b jets; b) c jets; c)light jets. . . . . . . . . . . . 56

5.8 Comparison between Pythia and Herwig for the secondary vertex mass templates for: a) b jets; b) c jets; c)light jets. . . . . 57

5.9 Plots showing predicted histograms from TFractionFitter and estimated $b$ contributions overlaid onto the spectrum used for data and Monte Carlo samples. The $p_{t}^{\text {rel }}$ spectrum is used for $F_{b}^{\text {jet }}$. The secondary vertex mass of the tagged electron jets is used for $F_{b}^{t a g}$. . . . . . . . . . . . . . . . . . 60

$5.10 \epsilon_{b}$ as a function of a) Jet Et, b) $\Delta \phi$, c) dijet mass and d)jet $\eta$. For the $\eta$ plots only the data are fitted. . . . . . . . . . . . . . 62

5.11 True $\epsilon_{b}$, found using HEPG information, as a function of $\eta$ for a) Herwig and b) Pythia Monte Carlo samples. . . . . . . . 63

5.12 True $\epsilon_{b}$, found using HEPG information, as a function of $\eta$ for a Pythia Monte Carlo sample with improved detector simulation. 63

5.13 True $\epsilon_{b}$, found using HEPG information, as a function of jet $E_{t}$ for a) Herwig and b) Pythia Monte Carlo samples. . . . . . 64

5.14 True $\epsilon_{b}$, found using HEPG information, as a function of jet $E_{t}$ for HEPG filtered and unfiltered samples. . . . . . . . . . . 65

5.15 Number of tracks with $p_{t}>1 \mathrm{GeV}$ within a b jet for unfiltered and HEPG electron filtered events, all histograms are normalised to unit area. . . . . . . . . . . . . . . . . 65

5.16 Number of good tracks within a $b$ jet for unfiltered and $H E P G$ electron filtered events, all histograms are normalised to unit

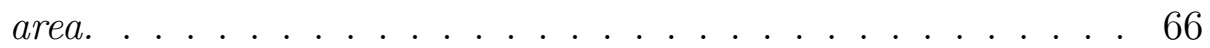

5.17 Number of tracks with $p_{t}>1 \mathrm{GeV}$ within a jet for HEPG filtered Monte Carlo and electron triggered and jet triggered datasets. Histograms are normalised to unit area. . . . . . . . 66

5.18 Correction factor as a function of jet $E_{t}$ to account for the difference in unfiltered and filtered Monte Carlo samples. The line represents a fit to a constant function, the fit parameters are shown in the top right corner. . . . . . . . . . . . . . . 67

5.19 SECVTX tagging efficiency in data, together with the fit and corresponding error. . . . . . . . . . . . . . . . . . 68 
6.1 Distributions of $E_{t}^{\text {cor }}$ (top), $\phi$ (middle) and $\eta$ (bottom) for the leading tagged jet (left) and second tagged jet (right). All Monte Carlo histograms are normalised to the number of events in data. . . . . . . . . . . . . . . . . . 74

6.2 Distributions of $E_{t}^{c o r}$ for a) the leading tagged jet and b) the second tagged jet. In both cases the first bin is excluded from the Monte Carlo normalisation. . . . . . . . . . . . . . 75

6.3 Comparisons between data and a) Pythia, b) Herwig and c) $M C @ N L O$ for the $\Delta \phi$ distribution. Monte Carlo histograms are normalised to the number of events in data. . . . . . . . . 76

6.4 The difference in $E_{t}$ between geometrically matched hadron (Had) and corrected calorimeter (Cal) jets, shown as a function of hadron jet $E_{t} . \ldots \ldots \ldots \ldots$. . . . . . . . . . 77

6.5 Profile plot showing the difference in $E_{t}$ between geometrically matched hadron (Had) and calorimeter (cal) jets, shown as a function of hadron jet $E_{t} . \quad \ldots \ldots \ldots$. . . . . . . . . . . . . . 79

6.6 Acceptance as a function of: a) the leading jet $E_{t}$; b) the dijet invariant mass of the leading jet and second jet; $c) \Delta \phi$ between the leading jet and second jet. The systematic uncertainties are discussed in section |6.4.1. . . . . . . . . . . . . . . . . . . 81

6.7 Secondary vertex mass spectra used for fits to find purity of the data sample. . . . . . . . . . . . . . . . . . . 84

6.8 The secondary vertex mass spectrum in data with the predicted Monte Carlo histogram and estimated $b$ contribution overlaid for the a) Leading tagged jets and b) Second tagged jets. . . . . 85

6.9 The secondary vertex mass spectrum in data with the predicted Monte Carlo histogram and estimated b contribution. . . . . . 85

7.1 The differential cross section as a function of leading jet $E_{t}$, shown on both linear(left) and log(right) scales. The line represents the systematic uncertainty which is described in section 7.4. . . . . . . . . . . . . . . 9 90

7.2 The differential cross section as a function of the invariant mass of the two tagged jets, shown on both linear(left) and $\log$ (right) scales. The line represents the systematic uncertainty which is described in section 7.4. . . . . . . . . . . 90

7.3 The differential cross section as a function of the azimuthal angle $\Delta \phi$ between the tagged jets, shown on both linear(left) and $\log$ (right) scales. The line represents the systematic uncertainty which is described in section 7 7.4. . . . . . . . . . . . 91 
7.4 The $E_{t}$ of the leading b jet in Pythia, Herwig and MC@NLO data samples. All plots are normalised to the integrated luminosity of the Pythia sample. . . . . . . . . . . . . . . . 92

7.5 The $E_{t}$ of the leading b hadron in Pythia, Herwig and MC@NLO data samples, all normalised to the integrated luminosity of the Pythia sample. a) For events containing two b hadrons in the central region where one has $E_{t}>30 \mathrm{GeV}$ and the other has $E_{t}>20 \mathrm{GeV}$. b) For events containing two b hadrons in the central region where one has $E_{t}>25 \mathrm{GeV}$ and the other has $E_{t}>15 \mathrm{GeV} . \ldots \ldots \ldots . \ldots . \ldots . \ldots 93$

$7.6 \quad$ The $E_{t}$ of the leading b jet for Pythia and $M C @ N L O+J I M M Y$ normalised to the integrated luminosity of the Pythia sample. . 94

7.7 The differential cross section as a function of a) leading jet $E_{t}$, b) $\Delta \phi$ and c) $M_{b \bar{b}}$ for data and MC@NLO+JIMMY predictions. 95

B.1 Schematic diagram showing how thet $a_{E M}$ and thet $a_{H A D}$ are different if the interaction vertex is shifted from the centre. . . 108

B.2 The difference between using at 60:40 ratio to split the energy for a particle and using a Gaussian distribution to split the energy for a) an interaction vertex located at 0 and b) an interaction vertex located $20 \mathrm{~cm}$ from 0 in the $z$ direction. . . . 109

C.1 Profile plot showing hadron (Had) jet $E_{t}$ divided by corrected calorimeter (cor Cal) jet $E_{t}$, as a function of the hadron jet $E_{t}$. The solid line represents a fit to a constant function, the fit parameters are shown in the top right corner. . . . . . . . . . 110

C.2 Profile plots showing hadron (Had) jet $E_{t}$ divided by corrected calorimeter (cor Cal) jet $E_{t}$, as a function of the hadron jet $E_{t}$, for: a) b-jets; b) c-jets; c) gluon-jets; d) uds-jets. The solid line represents a fit to a constant function, the fit parameters are shown in the top right corner of each plot. . . . . . . . . . . 111

C.3 Profile plots showing the ratio of hadron (Had) jet $E_{t}$ to corrected calorimeter (cor Cal) jet $E_{t}$, for gluon- and uds-jets combined, when the relative energy correction is adjusted by $+1 \sigma$ (left) and $-1 \sigma$ (right). The solid line represents a fit to a constant function, the fit parameters are shown in the top right corner. . . . . . . . . . . . . . . . 112 
C.4 Profile plots showing the ratio of hadron (Had) jet $E_{t}$ to corrected calorimeter (cor Cal) jet $E_{t}$, for gluon- and uds-jets combined, when the jet energy scale correction is adjusted by $+1 \sigma$ (left) and $-1 \sigma$ (right). The solid line represents a fit to a constant function, the fit parameters are shown in the top right corner. . . . . . . . . . . . . . . 112

C.5 Profile plots showing the ratio of hadron (Had) jet $E_{t}$ to corrected calorimeter (cor Cal) jet $E_{t}$, for gluon- and uds-jets combined, when the multiple interaction correction is adjusted by $+1 \sigma$ (left) and $-1 \sigma$ (right). The solid line represents a fit to a constant function, the fit parameters are shown in the top right corner. . . . . . . . . . . . . . . . . 113

C.6 Profile plots showing the ratio of hadron (Had) jet $E_{t}$ to corrected calorimeter (cor Cal) jet $E_{t}$, for gluon- and uds-jets combined, when the absolute energy correction is adjusted by $+1 \sigma$ (left) and $-1 \sigma$ (right). The solid line represents a fit to a constant function, the fit parameters are shown in the top right corner. . . . . . . . . . . . . . . . . . 113

C.7 Profile plots showing hadron (Had) jet $E_{t}$ divided by corrected calorimeter (cor Cal) jet $E_{t}$, as a function of the hadron jet $E_{t}$, for: a) non semi-leptonic $b$ decay; $b$ ) $b$ decay involving an electron; c) b decay involving a muon; d) b decay involving $a$ tau. The solid line represents a fit to a constant function, the fit parameters are shown in the top right corner. . . . . . . . . 114 


\section{Chapter 1}

\section{Introduction}

The Standard Model of particle physics was developed in the 1960s and describes the fundamental particles and their interactions in nature. The model consists of a set of gauge theories and is described in chapter 2, It has been extremely successful since its development and many experimental measurement have been found to be consistent with Standard Model predictions.

The analysis described in this thesis measures the cross section for b quark production, one of the fundamental particles within the Standard Model, in proton-antiproton $(p \bar{p})$ collisions. Data are collected using the CDF detector, which is described in chapter 3 . The measurement is made by identifying jets associated to b quarks through secondary vertex tagging. Chapter 4 describes the algorithm used to find jets and secondary vertex tagging is explained in chapter 5. The data samples used in the measurement and event selection procedure are described in chapter 6. Finally the cross section calculations and the conclusions are detailed in chapters 7 and 8 , respectively.

Previous measurements of the inclusive b quark cross section, discussed in section 2.6, have shown a discrepancy between data and Next to Leading 
Order predictions. Although this has since been reduced, a confirmation of the measured discrepancy could signal "new" physics. The analysis also provides a good insight into b quark production mechanisms and is a strong test for the Standard Model. By using jets rather than a hadronic decay channel for the measurement there is no dependence on branching fractions that often need to be measured experimentally. The requirement of two b jets within the pseudo-rapidity region $|\eta|<1.2$ picks out Leading Order production mechanisms and allows a specific part of QCD to be tested. 


\section{Chapter 2}

\section{b Quark Physics in $p \bar{p}$ Collisions}

\subsection{The Standard Model}

The Standard Model consists of a set of gauge theories describing the strong, weak and electromagnetic interactions which are mediated by integral spin gauge bosons. The model predicts all matter is made up of spin $\frac{1}{2}$ fermions and anti-fermions which are listed in table 2.1. The leptons only interact via the electromagnetic and weak interactions, mediated by the photon and $\mathrm{W} / \mathrm{Z}$ bosons. Quarks can also interact via the strong interaction, which is mediated by the gluons. The Higgs Mechanism [1] is responsible for the masses of the particles, which results in the addition of the scalar Higgs boson.

Within the Standard Model the electromagnetic and weak interactions are united in electroweak theory [2], this is briefly described in section 2.2 . The theory of Quantum Chromodynamics (QCD) is used to describe the strong interaction [3, 4, 5] and is used to calculate cross sections for the production of b quarks. QCD is described in detail in section 2.3 and the various 


\begin{tabular}{|c|c|c|c|c|}
\hline Туре & & & & Electric Charge \\
\hline \multirow{6}{*}{ Fermions } & \multicolumn{3}{|c|}{ Quarks } & \multirow[b]{2}{*}{$+2 / 3$} \\
\hline & $\mathrm{u}$ & $\mathrm{c}$ & $\mathrm{t}$ & \\
\hline & $\mathrm{d}$ & $\mathrm{s}$ & $\mathrm{b}$ & $-1 / 3$ \\
\hline & \multicolumn{3}{|c|}{ Leptons } & \\
\hline & e & $\mu$ & $\tau$ & -1 \\
\hline & $\nu_{e}$ & $\nu_{\mu}$ & $\nu_{\tau}$ & 0 \\
\hline \multirow{5}{*}{ Bosons } & \multicolumn{3}{|c|}{$\gamma$} & 0 \\
\hline & \multicolumn{3}{|c|}{ Z } & 0 \\
\hline & \multicolumn{3}{|c|}{$\mathrm{W}^{ \pm}$} & \pm 1 \\
\hline & \multicolumn{3}{|c|}{ Gluon } & 0 \\
\hline & \multicolumn{3}{|c|}{ Higgs } & 0 \\
\hline
\end{tabular}

Table 2.1: The fundamental particles within the Standard Model. Each fermion has an associated antiparticle which has opposite electric charge. 
production mechanisms for b quarks are discussed in section 2.4. Section 2.5 describes Monte Carlo event generators which incorporate QCD calculations for scattering processes with models for hadronisation and simulate particle decays in order to simulate $p \bar{p}$ collisions.

\subsection{Electroweak Theory}

Electroweak theory is a gauge theory for the $S U(2) \times U(1)_{Y}$ group, where $\mathrm{SU}(2)$ is the gauge group for the weak interaction. The $U(1)_{Y}$ group, however, is not the gauge group of the electromagnetic interactions but of hypercharge, this is denoted by the subscript $\mathrm{Y}$. The weak interaction only interacts with left handed quarks and leptons. These form doublets which are represented by the columns in table 2.1. The right handed components are singlets which are unaffected by the weak interaction.

The gauge bosons of the group ${ }^{1}\left(\mathrm{~B}, \mathrm{~W}^{1}, \mathrm{~W}^{2}\right.$ and $\left.\mathrm{W}^{3}\right)$ are all massless. The masses of the physically observed $\mathrm{W}^{ \pm}$and $\mathrm{Z}$ bosons are generated through the Higgs mechanism. A scalar Higgs field is introduced in order to break the symmetry of the group spontaneously. The coupling of the gauge bosons with the Higgs field results in the massive $W^{ \pm}$bosons and the $\mathrm{Z}$ boson. It also results in the entangling of the $\mathrm{SU}(2)$ and $\mathrm{U}(1)$ groups as the $\mathrm{Z}$ boson and the photon are linear combinations of the massless $\mathrm{B}$ and $\mathrm{W}^{3}$ bosons. This is shown in equations 2.1 and 2.2, where $\theta_{W}$ is the weak mixing angle.

\footnotetext{
${ }^{1}$ The $U(1)_{Y}$ group has one generator and the $\mathrm{SU}(2)$ group has three generators.
} 


$$
\begin{aligned}
& Z=\cos \theta_{W} W^{3}-\sin \theta_{W} B \\
& \gamma=\cos \theta_{W} B+\sin \theta_{W} W^{3}
\end{aligned}
$$

The weak eigenstates of the quarks are superpositions of the mass eigenstates of the physical quarks. This means quark transitions across generations are possible via the weak interaction. The mixing of the mass and weak eigenstates is described by the Cabibbo-Kobyashi-Maskawa (CKM) matrix 6].

\subsection{Quantum Chromodynamics}

QCD is a non-abelian gauge theory of the $\mathrm{SU}(3)$ group. The non-abelian nature means the gauge mediators interact with each other at three-point and four point vertices. Eight gauge mediators (bosons) have to be introduced in order to preserve local gauge invariance, these are the gluons. The quarks are described by the gauge fields $\psi_{i}$, where i runs from 1 to 3 and represents the colour quantum number.

For each quark flavour the basic QCD Lagrangian is given by:

$$
\begin{gathered}
\mathcal{L}=-\frac{1}{4} F_{\mu \nu}^{a} F^{a \mu \nu}+i \bar{\psi}\left(\gamma^{\mu} \mathbf{D}_{\mu}-m \mathbf{I}\right) \psi \\
F_{\mu \nu}^{a}=\partial_{\mu} A_{\nu}^{a}-\partial_{\nu} A_{\mu}^{a}-g_{s} f^{a b c} A_{\mu}^{b} A_{\nu}^{c} \\
\mathbf{D}_{\mu}=\partial_{\mu} \mathbf{I}+i g_{s} \mathbf{T}^{a} A_{\mu}^{a}
\end{gathered}
$$

where $\psi$ and $A^{a}$ are the quark and gluon fields. I is the unit matrix and $\mathbf{T}^{a}$ are the generator matrices. $f^{a b c}$ define the Lie algebra [7] of the group 
and are known as "structure constants". $g_{s}$ is the gauge coupling constant and $m$ is the mass of the quark. The indices $a, b$ and $c$ run from 1 to 8 and represent the eight mediators. The Lagrangian defines the quark and gluon interactions and their propagators.

The Feynman rules for the propagators and interaction vertices are determined directly from the Lagrangian. The rules are only useful within the context of perturbation theory, which requires the strength of the coupling to be small. This however is not a problem since the coupling constant varies as a function of four-momentum scale (distance).

\subsubsection{Asymptotic Freedom}

The coupling constant is more commonly represented as $\alpha_{s}$ where:

$$
\alpha_{s}=\frac{g_{s}^{2}}{4 \pi}
$$

Loop corrections made to the gluon propagator introduce integrals that diverge to infinity, these are known as "UV" divergences. These are renormalised by subtracting at some renormalisation scale $\mu$, which appears inside a logarithm for the renormalised quantities. For interactions at a fourmomentum scale $\mathrm{Q}$, where $Q>>\mu$, this results in the coupling constant depending on a logarithmic term of $Q^{2} / \mu^{2}$ which obeys the differential equation:

$$
\frac{\partial \alpha_{s}\left(Q^{2}\right)}{\partial \ln \left(Q^{2}\right)}=\beta\left(\alpha_{s}\left(Q^{2}\right)\right)
$$

$\beta$ has a perturbative expansion given by equation 2.8 and $\beta_{0}$, is calculated using the number of colours, $N_{c}$, and number of flavours whose mass threshold 
is below the four-momentum scale, $n_{f}$.

$$
\begin{gathered}
\beta(\alpha)=-\beta_{0} \alpha^{2}+\mathcal{O}\left(\alpha^{3}\right)+\ldots \\
\beta_{0}=\frac{11 N_{c}-2 n_{f}}{12 \pi}
\end{gathered}
$$

The negative sign of $\beta$ results in the coupling strength falling as a function of four-momentum scale, this is known as "asymptotic freedom". This means that at large four-momentum scales it is possible to calculate the scattering cross sections of quarks and gluons using perturbation theory. The converse argument explains why free quarks and gluons are not observed in nature. A small four-momentum scale is equivalent to a large distance where the coupling strength gets stronger. So as quarks are pulled further from each other more and more energy is required to overcome the binding. This results in quarks and gluons being bound inside hadrons, this phenomenon is known as "confinement".

As quarks and gluons involved in scattering processes move apart they form themselves into hadrons through a process called "hadronisation". This happens at a distance where the coupling constant is strong, therefore it is not possible to use perturbation theory to describe it. Instead models are used to represent hadronisation and are implemented in Monte Carlo generators, these models are discussed in section 2.5.2. Hadronisation often results in multiple hadrons being produced, all moving in the original quark direction. These are known as "jets" and are the experimental signature of quarks and gluons. 


\section{4 b Quark Production Mechanisms}

The Leading Order and Next to Leading Order production mechanisms in $p \bar{p}$ collisions can be categorised into three classes: flavour creation; flavour excitation; and gluon splitting. Figure 2.1 shows Feynman diagrams for each class. In the flavour creation category the $b \bar{b}$ pair are produced via the Leading order processes of gluon-gluon fusion or $q \bar{q}$ annihilation. During flavour excitation gluon fragmentation to a $b \bar{b}$ pair occurs in the initial state and results in one of the $\mathrm{b}$ quarks being scattered from the initial state to the final state by a gluon or light quark. The final category contains events where the $b \bar{b}$ pair are produced in the final state during the fragmentation process of a gluon or light quark. The flavour excitation and gluon splitting processes are only possible at Next to Leading Order.

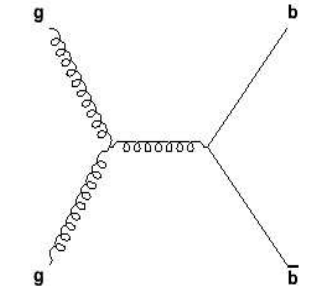

(a) Flavour Creation

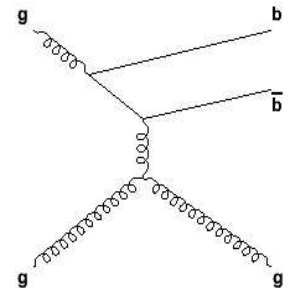

(b) Flavour Excitation

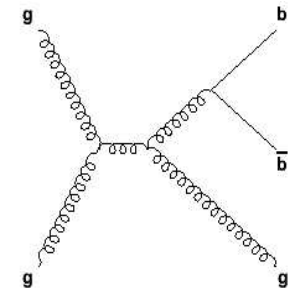

(c) Gluon Splitting

Figure 2.1: Example Feynman diagrams for: a) flavour creation; b) flavour excitation; c) gluon splitting.

Although events from the various categories cannot be fully separated, there are various kinematic characteristics which allow some distinction to be made [8]. Figure 2.2 is taken from reference [8], it shows the differential 
cross section as a function of the azimuthal angle, the angle in the plane transverse to the beam direction, between the $b \bar{b}$ pair. Unlike flavour excitation and gluon splitting which both tend to have a flat distribution, the flavour creation process results in the $b \bar{b}$ pair coming out predominantly back to back in the transverse plane. By requiring two b jets and measuring the cross section as function of the angle between them it is possible to qualitatively predict which process is dominant in the sample.

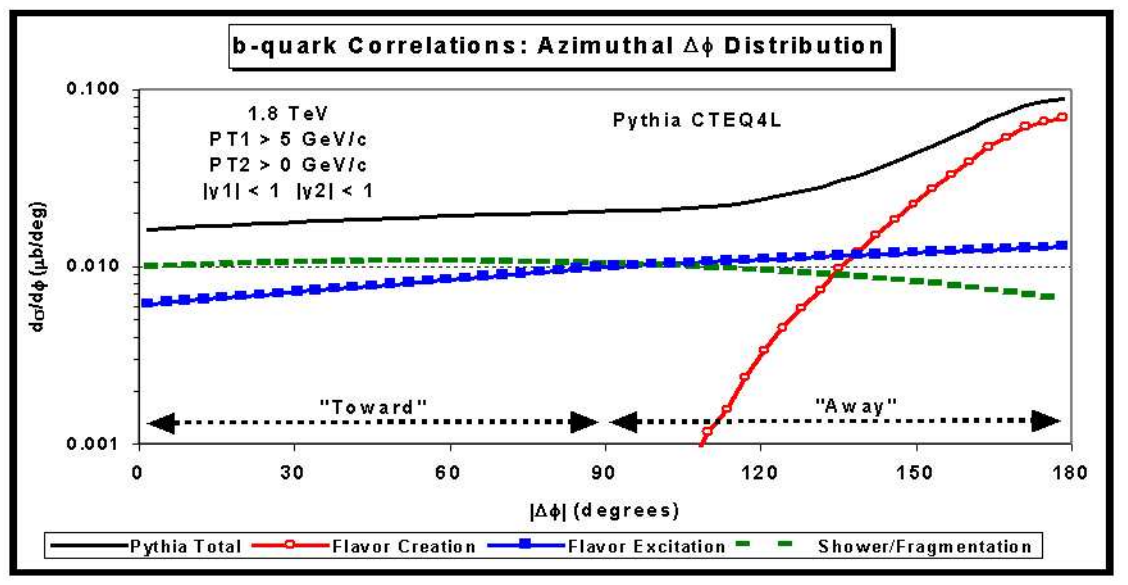

Figure 2.2: Differential cross section predictions as a function of the azimuthal angle between the $b \bar{b}$ pair for the various categories, taken from reference [8].

\subsection{Monte Carlo Event Generators}

Monte Carlo event generators are used in a variety of ways in the analysis, from finding characteristic spectra for variables associated with b quarks to finding predictions for the cross section of $b \bar{b}$ jet production. Pythia [9] and 
Herwig [10] Monte Carlo generators incorporate the QCD scattering processes at Leading Order, calculated using perturbation theory, radiation of gluons from the incoming protons and outgoing partons after the scattering, hadronisation and decay of heavy objects in order to simulate $p \bar{p}$ collisions at Tevatron energies. The MC@NLO [11] Monte Carlo generator calculates QCD scattering processes at Next to Leading Order using perturbation theory and links to Herwig for the simulation of hadronisation and particle decays.

In terms of the b quark production mechanisms discussed in section 2.4 Pythia and Herwig calculate the flavour creation processes explicitly using perturbative QCD but the flavour excitation and gluon splitting processes are only approximated from the initial state and final state radiation simulation. MC@NLO instead calculates all the processes at Next to Leading Order using perturbation theory.

\subsubsection{Parton Distribution Functions (PDFs)}

The quarks and gluons that form hadrons are known as "partons". During $p \bar{p}$ collisions it is the constituent partons that are involved in any hardscattering process. The parton distribution functions describe the fraction, $x$, of longitudinal momentum carried by the various partons in the proton. These are used by the Monte Carlo generators to calculate the scattering cross sections and to simulate initial state gluon radiation.

The change in PDFs as a function of the momentum scale is described by the DGLAP equations [12]. This allows PDFs found at one momentum scale to be used for other momentum scales. PDFs are currently determined 
by a global fit to a wide range of experimental data that are sensitive to different regions in $x$. These fits are made by the CTEQ [13] and MRST [14] collaborations. The experiments used to calculate the PDFs include fixed target experiments based at SLAC, CERN and FNAL and deep inelastic scattering data from electron-proton collisions at HERA. In this analysis the CTEQ 5l PDFs are used with both Pythia and Herwig and the MRST 1999 PDFs are used with MC@NLO.

\subsubsection{Hadronisation}

Hadronisation is not calculable using perturbation theory and models are used by the Monte Carlos to simulate it. Pythia uses the Lund String Model [15] and Herwig uses the Cluster Model [16].

The Lund String Model is based on confinement and is best explained using $q \bar{q}$ production. As the $q$ and $\bar{q}$ move apart from each other a colour string connecting the two is stretched causing its potential energy to rise. Eventually the string has enough potential energy that it can break and produce a new quark-antiquark pair, $q^{\prime} \bar{q}^{\prime}$. Quantum mechanical tunnelling is used to produce the quark-antiquark pairs. The tunnelling probability is given by equation 2.10, where $m$ is the mass of the quark, $p_{t}$ is its transverse momentum and $\kappa$ is the energy density of the string. It results in a suppression of heavy flavour quarks with the ratio $\mathrm{u}: \mathrm{d}: \mathrm{s}: \mathrm{c} \approx 1: 1: 0.3: 10^{-11}$. When the string breaks the system splits into two colour-singlet subsystems $q \bar{q}^{\prime}$ and $q^{\prime} \bar{q}$, where each subsystem is connected by a string. If the string has a high enough invariant mass, further string fragmentation is possible, which 
continues until only on-mass-shell hadrons remain.

$$
p(\text { tunnelling })=\exp \left(-\frac{\pi m^{2}}{\kappa}\right) \exp \left(-\frac{\pi p_{t}^{2}}{\kappa}\right)
$$

The Cluster Model uses a process known as "preconfinement". In the period after the hard scatter, where $\alpha_{s}$ is still small, perturbative QCD can be used to generate a parton shower by radiating gluons. The gluons can be considered as colour-anticolour pairs and are non-perturbatively split into quark-antiquark (diquark-diantiquark) pairs after the parton shower. By following the colour structure of the parton shower the quarks and gluons can be organised into colour-singlet clusters, this is defined as preconfinement. The process is shown schematically in figure 2.3 . These clusters are then fragmented into hadrons. If a cluster is too light to decay into hadrons it is taken to represent the lightest single hadron of its flavour and its mass is shifted to the appropriate value by exchanging four-momentum $2^{2}$ with a neighbouring cluster. Clusters able to decay to two hadrons do so isotropically, in the centre of mass frame of the cluster. A random flavour $f$ is chosen from $\mathrm{u}, \mathrm{d}, \mathrm{s}, \mathrm{c}$ and the six diquark combinations of $\mathrm{u}, \mathrm{d}$ and $\mathrm{s}$. Thus a cluster $f_{1} \bar{f}_{2}$ is split into $f_{1} \bar{f}$ and $\bar{f}_{2} f$ and the hadrons corresponding to these flavours are chosen at random from a list of available hadrons. The choice is only accepted in accordance with the density of states for that channel, otherwise $f$ is rejected and the procedure repeated. An iterative processes is used to hadronise all the clusters.

\footnotetext{
${ }^{2}\left(\mathrm{E}, p_{x}, p_{y}, p_{z}\right)$ where $\mathrm{E}$ is the energy and $p_{x}, p_{y}, p_{z}$ are the $x, y, z$ components of the momentum.
} 


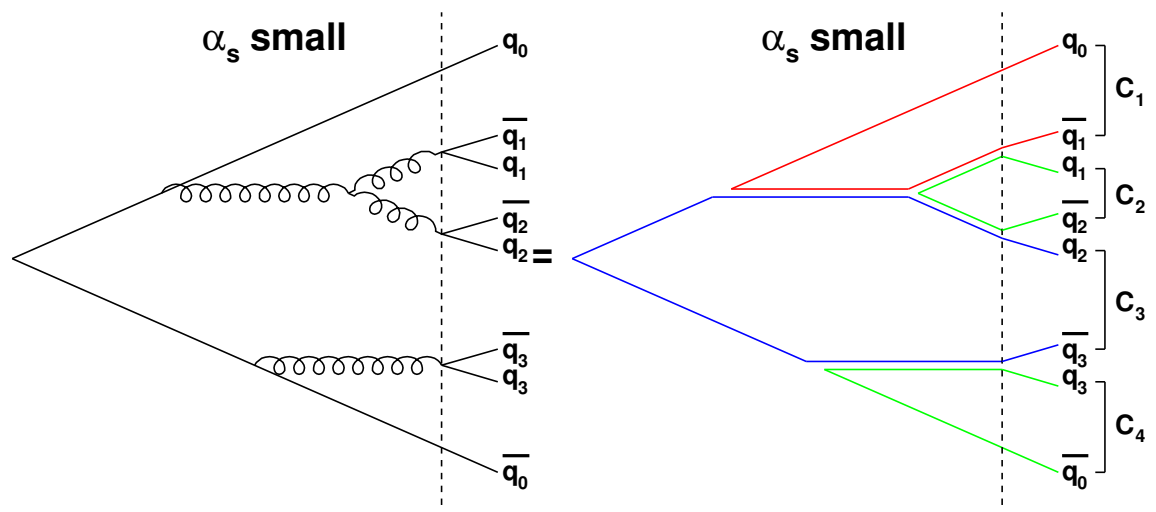

Figure 2.3: Schematic diagram showing how clusters $\left(C_{1}, C_{2}, \ldots\right)$ are formed using preconfinement by following the colour flow. The dashed line represents the point at which $\alpha_{s}$ becomes large.

\subsection{3 b quark decay}

b quarks have an average lifetime of $1.564 \pm 0.014$ ps [17], so they form hadrons before decaying. The decay of the b quark within the hadron proceeds via the weak interaction, with the emission of a $\mathrm{W}$ boson. The quark that the $\mathrm{b}$ decays to is combined with the remaining quark contents of the hadron to form a new hadron. If the $\mathrm{W}$ boson decays to two quarks these are combined to form a second hadron. To form higher multiplicity decays the hadronisation model is used to form additional hadrons. The b quarks predominantly decay to the c quark, this also decays weakly and the same procedure is used.

Since the decay of the b quarks occurs some time after the initial interaction, the vertex at which the decay occurs is well separated. This allows jets originating from $\mathrm{b}$ quark hadronisation to be identified and forms the basis of this analysis. 


\subsection{Previous Measurements of $b$ Production}

The first measurements of the b quark cross section in $p \bar{p}$ collisions were made by the UA1 collaboration [18]. The centre-of-mass energy of the collisions was $630 \mathrm{GeV}$. Samples containing muons were used to extract the b quark cross section and the measurement showed agreement with Next to Leading Order QCD predictions. Both the CDF [19] and D0 [20] collaborations then measured the cross section at a centre-of-mass energy of $1.8 \mathrm{TeV}$. The analyses used muons and $J / \psi$ s to identify b hadrons and showed an excess in the measured rate of $\mathrm{b}$ quark production by a factor of two or more when compared to Next to Leading Order calculations. The H1 [21] collaboration at HERA also measured an excess in the rate of $\mathrm{b}$ quark production in electronproton scattering, again muon data samples were used to extract the cross section. The results are summarised in table 2.2.

\begin{tabular}{|c|c|c|c|}
\hline Collaboration & $\min \mathrm{p}_{t}^{b}(\mathrm{GeV} / \mathrm{c})$ & $\sigma_{b}$ & Discrepancy \\
\hline UA1 (1991) & 6 & $19.3 \pm 7($ exp. $) \pm 9($ th. $) \mu b$ & Agrees \\
\hline CDF (1992) & 8.5 & $10.5_{-5.1}^{+5.0} \mu b$ & $+1.7 \sigma$ \\
\hline CDF (1993) & 21 & $295 \pm 21 \pm 75 n b$ & $+2.1 \sigma$ \\
\hline D0 (1995) & 6 & $8.0 \pm 2 \mu b$ & $+1.5 \sigma$ \\
\hline D0 (1996) & 9.9 & $2.0 \pm 0.5 \mu b$ & $+2.0 \sigma$ \\
\hline H1 (2000) & - & $206 \pm 19_{-40}^{+46} n b$ & $+2.6 \sigma$ \\
\hline \hline
\end{tabular}

Table 2.2: Summary of previous measurements of the inclusive b quark production cross section.

One of the inherent problems with the analyses was that they had measured the cross section using b hadrons (hadrons containing b quarks) and 
quoted the results in terms of the quark kinematics rather than those of the hadron. The fraction of the $\mathrm{b}$ quarks momentum carried by the $\mathrm{b}$ hadron is parametrised using fragmentation functions, such as those produced by Peterson et al. [22]. Using fragmentation functions the measured hadron cross sections are deconvoluted to extract a b quark cross section ie. going from the hadron level to the quark level where:

- The hadron level is where the kinematics are described in terms of the hadrons that are measured by the experiment.

- The quark level is where the hadron kinematics are adjusted in order to quote results in terms of the quarks from which the hadrons originated.

Cacciari and Nason reevaluated the deconvolution of data from the hadron level to the quark level [23]. They also improved theoretical calculations [24] and showed the discrepancy could be reduced. They conclude that measurements made should not be deconvoluted to the quark level but should be kept at the hadron level, ie. the b jet cross section should be measured rather than the b quark cross section. Taking this into consideration, in this analysis b jets are identified and the results are quoted with respect to the the jet kinematics, rather than the b hadrons within them or the b quarks that produced them. 


\section{Chapter 3}

\section{The Tevatron and the Collider Detector at Fermilab (CDF)}

The Tevatron is a circular $p \bar{p}$ accelerator with a radius of $1 \mathrm{~km}$. CDF is one of two multipurpose detectors located at the $p \bar{p}$ collision points and is used to detect the particles produced during the $p \bar{p}$ interactions.

Tevatron operations began in October 1985 at a centre-of-mass energy of $1.8 \mathrm{TeV}$ (Run I). Over $100 \mathrm{pb}^{-1}$ of data had been collected and analysed when Run I finished in 1996, resulting in over 100 published papers and the discovery of the top quark [25]. After the end of Run I major upgrades were performed on the Tevatron in order to increase the centre-of-mass energy to $1.96 \mathrm{TeV}$ and provide a higher instantaneous luminosity; CDF was also upgraded to improve the detector and accommodate the new running conditions. The new data-taking period, Run II, started in June 2001 and so far over $500 \mathrm{pb}^{-1}$ of data have been collected; $65 \mathrm{pb}^{-1}$ of Run II data are used for this analysis.

The remainder of this chapter describes the accelerator and the CDF 
detector in more detail.

\subsection{Accelerating the Protons and Antipro- tons}

A series of accelerators are required to accelerate the protons and antiprotons to $980 \mathrm{GeV}$. The system used contains: a Cockcroft-Walton accelerator; a linear accelerator (the Linac); five synchrotrons (the Booster, the Main Injector, the Tevatron, the Debuncher and the Accumulator); and a storage ring (the Recycler). Figure 3.1 is a schematic diagram of the accelerator system showing how the various accelerators are connected.

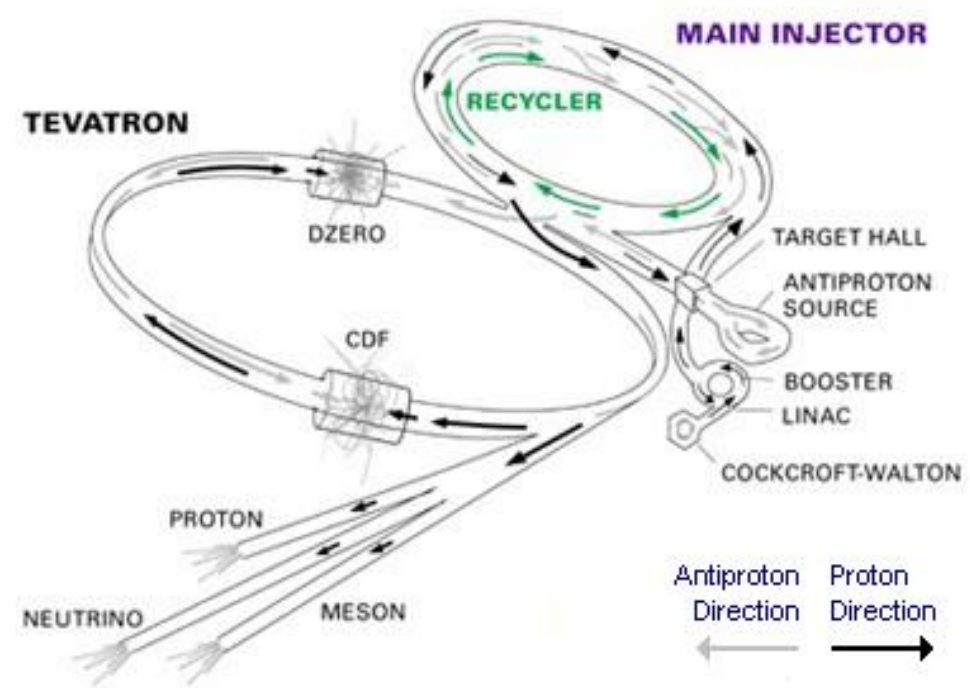

Figure 3.1: Schematic diagram of the accelerator system. 


\subsubsection{Protons}

Electrons are added to hydrogen atoms to produce $\mathrm{H}^{-}$ions which are accelerated to $750 \mathrm{KeV}$ in the Cockcroft-Walton accelerator - a multistage voltage multiplier providing a DC voltage of $750 \mathrm{kV}$ for acceleration. The Linac is used to accelerate the ions to an energy of $400 \mathrm{MeV}$ at which point they are passed through to the Booster. In the Booster the $\mathrm{H}^{-}$ions are stripped of their electrons by passing them through a thin carbon foil and the resulting protons are accelerated to energies of $8 \mathrm{GeV}$. The protons are boosted up to energies of $150 \mathrm{GeV}$ in the Main injector and then injected into the Tevatron where they are accelerated to $980 \mathrm{GeV}$.

\subsubsection{Antiprotons}

Every 1.5 seconds protons from the Main Injector are directed to a Nickel fixed target. The collisions produce bunches of antiprotons with a wide spread in energies, which are refined in the Debuncher. The refining process also results in the bunches being converted into a steady stream. The antiprotons come from the target with random positions and angles resulting in a "wide" beam and so are also stochastically cooled [26] in the Debuncher. The process is a feedback system designed to collimate the beam.

The antiprotons are passed through to the Accumulator where they are cooled further. As the name suggests the Accumulator accumulates successive pulses from the Debuncher and can store them for many hours. The $8 \mathrm{GeV}$ antiprotons in the Accumulator are passed to the Main Injector for acceleration to $150 \mathrm{GeV}$. Finally in the Tevatron they are accelerated to 980 $\mathrm{GeV}$ ready for collisions. 
The antiproton system also includes the Recycler which has two functions. Firstly, it acts as a post accumulator ring if there are too many antiproton stacks in the Accumulator. Secondly, it stores antiprotons that are left over after collisions in the Tevatron. Both of these functions help increase the instantaneous luminosity [27].

\subsection{The Collider Detector at Fermilab (CDF)}

The CDF detector is a multipurpose detector providing charged particle tracking, calorimetry and muon identification. The detector has both azimuthal and forward backward symmetry as shown in figure 3.2. Also labelled on the figure are the parts of the detector that have been kept from Run I, parts that have been upgraded and parts that are new for Run II. The superconducting solenoid enclosing the tracking system generates a 1.41 $\mathrm{T}$ magnetic field in the beam direction. Each of the systems highlighted in the schematic diagram is discussed in detail later in this section.

In the $\mathrm{CDF}$ coordinate system, shown in figure 3.3 , the proton direction defines the $\mathrm{z}$ axis, the polar $(\theta)$ and azimuthal $(\phi)$ angles related to the coordinate system are also shown on the diagram. The pseudo-rapidity $(\eta)$, defined in equation 3.1, is used instead of $\theta$ as it is invariant under boosts in the $\mathrm{z}$ direction.

$$
\eta=-\ln \left(\tan \left(\frac{\theta}{2}\right)\right)
$$




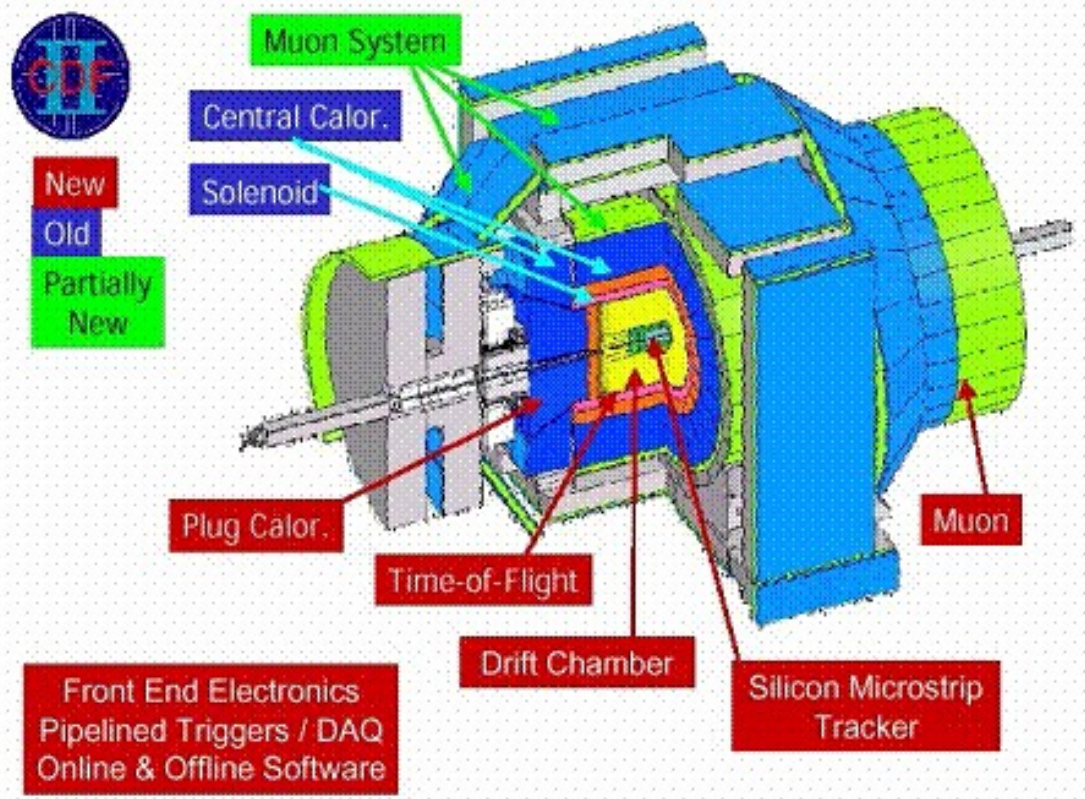

Figure 3.2: Schematic diagram of the CDF detector.

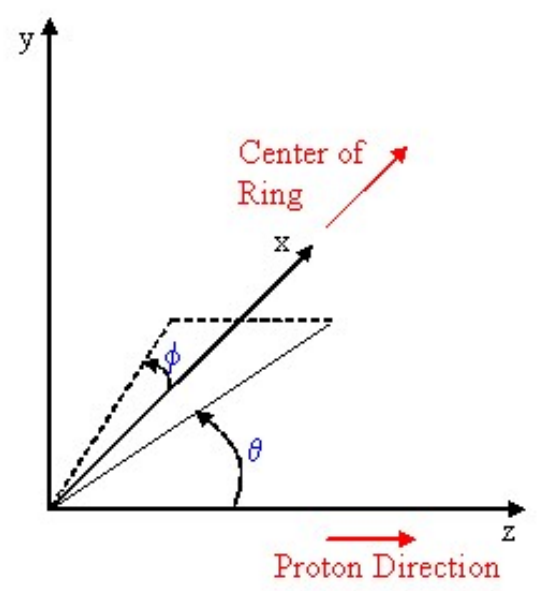

Figure 3.3: The CDF coordinate system. 


\subsubsection{Silicon Tracking System}

The silicon tracking system [28] is split into three subsystems: Layer 00; the Silicon Vertex Detector (SVX II); and the Intermediate Silicon Layers (ISL). The system consists of up to eight layers of silicon detectors and extends radially from $1.35 \mathrm{~cm}$ to $28 \mathrm{~cm}$. The length in the z direction ranges between $90 \mathrm{~cm}$ and $2 \mathrm{~m}$. Figure 3.4 shows the positions of each of the layers and how the detectors are arranged to provide full coverage for the region $|\eta|<2.0$.

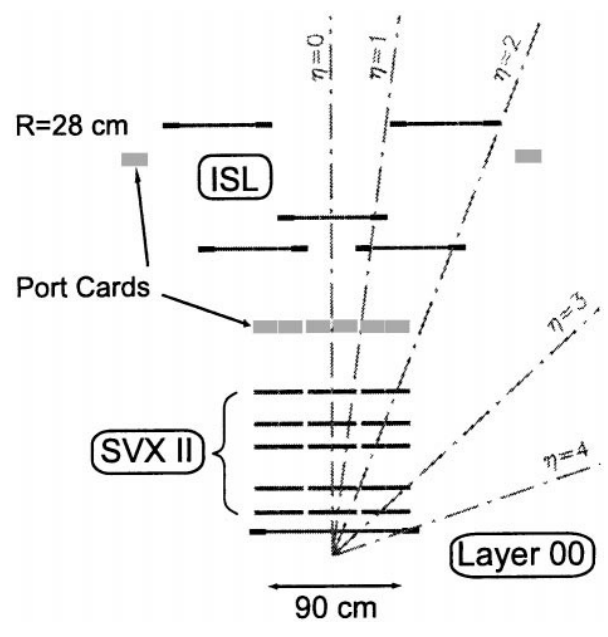

Figure 3.4: Arrangement of the silicon tracking system in the $r$-z plane.

The innermost subsystem, Layer 00, is supported by the beam pipe. It consists of one layer of single sided silicon modules. The strips on the modules are aligned parallel to the beam direction and provide measurements in the r- $\phi$ plane, known as "axial" hits.

Five radial layers of double sided silicon modules make up the SVX II, extending from $2.4 \mathrm{~cm}$ to $10.7 \mathrm{~cm}$. The subsystem is split into three barrels in the $\mathrm{z}$ direction, $29 \mathrm{~cm}$ in length. Each barrel is split into 12 azimuthal wedges and is supported by beryllium bulkheads at each end, which also 
contain the readout chips. On all five layers the silicon modules have strips parallel to the beam direction on one side to detect axial hits; on the other side, modules on Layer $0^{1}, 2$ and 4 have strips perpendicular to the beam direction which detect "stereo" hits, measurements in the r-z plane. The two remaining layers have their strips aligned at an angle of $+1.2^{\circ}$ (Layer 1) and $-1.2^{\circ}$ to the beam direction and detect "small angle stereo" hits, providing extra measurements in the r-z plane.

The ISL has one layer in the region $|\eta|<1.0$ at a radius of $22 \mathrm{~cm}$, and two layers at radii of $20 \mathrm{~cm}$ and $28 \mathrm{~cm}$ in the region $1.0<|\eta|<2.0$. Like the SVX II, the ISL modules are made up of double sided silicon wafers with one side detecting axial hits and the other side detecting small angle stereo hits.

The silicon system is essential for finding displaced vertices originating from b hadron decays; one of the main parameters used is the distance of closest approach to the primary vertex, the impact parameter $\left(d_{0}\right)$, which is typically large for tracks from b hadron decays. The impact parameter resolution of the silicon tracking as a function of transverse momentum $\left(p_{t}\right)$ is shown in figure 3.5 [29]. It is below $50 \mu \mathrm{m}$ for tracks with $p_{t}>1 \mathrm{GeV}$ and lower still if Layer 00 is used. The axial hit resolution of the system is $9 \mu \mathrm{m}$ [30]. In the region $|\eta|>1.2$, where the Central Outer Tracker coverage is incomplete, the silicon system can be used for stand-alone tracking and has a transverse momentum resolution of $\sigma_{p_{t}} / p_{t}^{2} \sim 0.4 \%$ [31].

\footnotetext{
${ }^{1}$ The layer numbering begins at 0 for the innermost layer and increases radially outwards.
} 


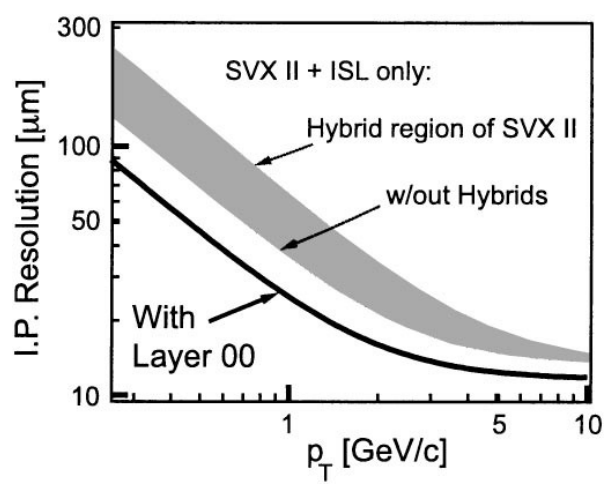

Figure 3.5: The impact parameter resolution as a function of $p_{t}$ for the silicon system.

\subsubsection{Central Outer Tracker (COT)}

The COT [32] is an open cell drift chamber located outside of the silicon system, it extends radially from $40 \mathrm{~cm}$ to $138 \mathrm{~cm}$ and provides full coverage in the region $|\eta|<1.2$. Radially the detector is split into eight "superlayers", each superlayer is segmented into "supercells" in $\phi$, with 12 sense wires per supercell. The supercells are arranged at an angle of $35^{\circ}$ to the radial direction. Alternating superlayers provide axial and stereo hit information, the sense wires for axial superlayers are aligned parallel to the beam direction and the stereo superlayers have sense wires aligned at an angle of $2^{\circ}$ to the beam direction. The gas used in the drift chamber is a 50:50 mixture of Argonne-Ethane and a small amount of Isopropyl alcohol.

The single hit position resolution of the COT is $180 \mu \mathrm{m}$ and the momentum resolution for charged particles is $\sigma_{p_{t}} / p_{t}^{2} \simeq 0.3 \%(G e V / c)^{-1}[31$. The combination of COT and silicon tracking has a momentum resolution of $\sigma_{p_{t}} / p_{t}^{2}<0.1 \%(\mathrm{GeV} / \mathrm{c})^{-1}$ 


\subsubsection{Time-of-Flight Detector (TOF)}

The TOF [33] is located outside the COT at a radius of $140 \mathrm{~cm}$ from the beam pipe and covers the region $|\eta|<1.0$. The detector consists of an array of 216 scintillator bars with photomultiplier tubes attached to each end. Amplitude information is measured and used to determine the time a particle crossed the scintillator. With a timing resolution of 100 ps the detector is able to identify kaons from pions with at least $2 \sigma$ separation for particles with momenta less than $1.6 \mathrm{GeV} / \mathrm{c} 33$.

\subsubsection{Calorimeters}

The calorimeters are located outside of the solenoid and provide coverage in the region $|\eta|<3.64$. The system is split into two regions: the central region, $|\eta|<1.2$, and the plug region, $1.2<|\eta|<3.64$. The calorimeter system is segmented into towers and arranged such that they point back to the nominal interaction point. The central calorimeters are split into towers that span $15^{\circ}$ in $\phi$ and 0.11 units in $\eta$. Towers in the plug region span either $15^{\circ}$ or $7.5^{\circ}$ in $\phi$ and various $\eta$ ranges.

The front section of each tower $2^{2}$ consists of layers of lead and scintillator, there are twenty three layers in total and a unit layer contains $4 \mathrm{~mm}$ of lead and $4.5 \mathrm{~mm}$ of scintillator. This section is known as the "Electromagnetic Calorimeter" [34] and is used primarily for energy measurements of photons and electrons; the thickness of the electromagnetic calorimeter is 21 radiation lengths and so these particles will deposit the majority of their energy here. The energy resolution for the central region is $\sigma(E) / E=13.5 \% / \sqrt{E_{t}}$ [31].

\footnotetext{
${ }^{2}$ The section that is radially closest to the beam pipe
} 
Behind this is the "Hadronic Calorimeter" [35], consisting of twenty three layers; a unit layer is made up of $25.4 \mathrm{~mm}$ of steel and $10 \mathrm{~mm}$ of scintillator. This is where high energy jets will deposit the majority of their energy, the energy resolution in the central region is $\sigma(E) / E=75 \% / \sqrt{E_{t}}$ [31].

Completing the calorimeter system are two proportional wire chambers. One is situated in front of the towers and is backed by $10 \mathrm{~mm}$ of scintillator. Known as the "Pre-Radiator" [31, the chamber is primarily used to identify conversion electrons. The second wire chamber, known as the "Shower Maximum Detector" [34] is embedded inside the towers at a distance equivalent to 6 radiation lengths, where the shower from electromagnetic particles will be at a longitudinal maximum. The detector is used to measure the profile of the shower and also its position. The position resolution is $2 \mathrm{~mm}$ for 50 GeV electrons [34].

\subsubsection{Muon Systems}

The calorimeter steel, magnet return yoke and additional steel shielding stops charged particles, other than muons, from reaching the muon detection chambers [31], which are located furthest from the beam pipe. These consist of drift chambers and scintillators and cover the region $|\eta|<2.0$. The chambers are able to detect muons that have a $p_{t}$ greater than $1.4 \mathrm{GeV}$ [31].

\subsubsection{Cherenkov Luminosity Counters (CLC)}

The CLC [36] are located inside the $3^{\circ}$ holes between the end plug calorimeters and the beam pipe. Each module consists of conical gas-filled Cherenkov counters, arranged around the beam pipe and pointing to the interaction 
region. The CLC measure the average number of interactions per beam crossing, which is used to calculate the luminosity [37]. The detectors also provide monitoring of the beam data for Tevatron physicists and luminosity monitoring for CDF operations.

\subsubsection{Triggering}

The collision and subsequent $p \bar{p}$ interaction rate at the Tevatron is much higher than the rate at which data can be stored to tape. CDF uses a trigger system to decide which events are kept. The trigger system is split into three levels, with more detector information available for each level. The level 1 and level 2 trigger decisions are made in custom hardware whereas level 3 decisions are made by software algorithms on a PC farm. Figure 3.6 shows a functional block diagram of the data acquisition process. Data from the detector are sent to the level 1 trigger system. If accepted, data are passed to the Level 2 buffers whilst the level 2 trigger system is making a decision, a process that takes on average $20 \mu \mathrm{s}[31$. If accepted at level 2, data are passed to the DAQ buffer. They are then sent to the Level 3 farm where the event is assembled, analysed and stored, if accepted. CDF uses trigger paths to specify which level 2 and level 3 triggers will be considered. Each level 2 trigger has a level 1 prerequisite and is only considered if this is met. Similarly each level 3 trigger has a level 1 and level 2 prerequisite. The trigger system is able to find objects such as jets, electrons, photons and muons. Trigger decisions are also made on variables such as missing transverse energy. Jet and electron trigger paths are used for this analysis and are described in more detail. 


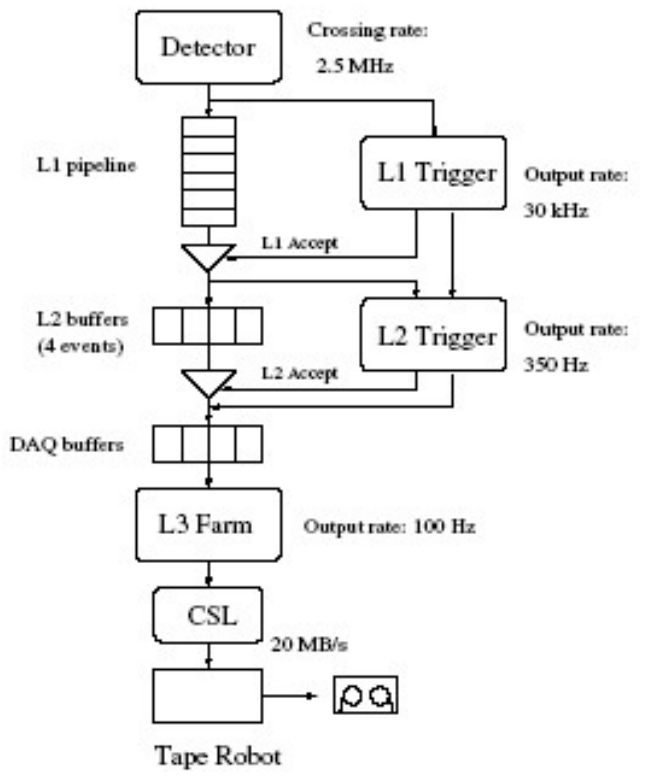

Figure 3.6: Functional block diagram of the data acquisition process.

The level 1 trigger uses information from the calorimeters, COT and muon chambers to make triggering decisions. It is split into three streams: one finds tracks, one finds calorimeter based objects and the third finds muons. The calorimeter stream is used for jet and electron trigger paths, it triggers on objects(electrons, photons and jets) consisting of single calorimeter towers. Electron triggers place cuts on the electromagnetic energy of objects for decision making and can also use tracks (found from the track stream) linked to the object. For jet triggers, cuts are placed on the total electromagnetic and hadronic energy of objects.

Events that are accepted by level 1 are passed, together with level 1 triggering information, to level 2. Jet trigger paths place cuts on the energy of clusters made during level 2 . The calorimeter is segmented into a $24 \times 24$ trigger tower array in $\eta-\phi$ space, each trigger tower is 0.2 by $15^{\circ}$. Two 
energy thresholds are set: a "seed" threshold, which is the minimum energy a tower needs to be considered as a seed for clustering; and a "shoulder" threshold, the minimum energy required for a tower to be considered as part of a cluster. The cluster finding algorithm scans the tower array and marks trigger towers above the seed or shoulder threshold. Starting from the seed tower closest to $\eta$ of zero, the algorithm looks for seed or shoulder towers in the four orthogonal neighbours. If a neighbour is found then the algorithm looks for seed or shoulder towers around that tower. The process continues until no adjacent towers are above the shoulder threshold, at which point the energy of all towers associated to the cluster is summed. The next unused seed tower is then used to find another cluster and the process continues until no more clusters are found. Electron trigger paths are able to use the extra information from the shower maximum detectors that is available at level 2 .

The level 3 trigger is a CPU farm made up of 256 nodes [38]. Events accepted at level 2 are passed to the DAQ buffers where the Event Builder assembles information for the entire event and passes it through to a level 3 processor node. On the node the event is fully reconstructed and level 3 filtering algorithms are run. The code used on the Level 3 processor is based on the offline reconstruction code. Jet finding algorithms described in chapter 4 are used for jet trigger paths.

\subsection{Offline Event Reconstruction}

Events accepted by level 3 are passed to the Consumer Server Logger (CSL) which sends data to a tape robot for writing. The CSL uses the level 3 decision to sort the data into three categories: events for data analysis; events 
for online monitoring and rare events for immediate analysis.

The raw data stored to tape are processed on a PC Farm [39] and split into datasets according to which trigger paths were satisfied. The offline reconstruction code used for the processing is written in the $\mathrm{C}++$ programming language and runs a set of algorithms that reconstruct track, electron, photon, muon and jet objects from the raw data. Calibration constants for the various detectors are stored in databases and used to correct detector data during offline reconstruction. 


\section{Chapter 4}

\section{Jets}

There are a variety of algorithms that can be used to define jets. Cone based algorithms use a cone of radius $\mathrm{R}$, calculated using equation 4.1, and effectively sum all "particles" (calorimeter towers, stable hadrons in Monte Carlo generators, partons in QCD calculations) that lie within the cone in order to define a jet; examples include the "Jet Clustering", "MidPoint" and "Seedless Cone" algorithms [40]. There are also algorithms that successively merge particles in order of increasing transverse momentum, these are known as " $K_{T}$ " algorithms [40].

$$
R=\sqrt{\eta^{2}+\phi^{2}}
$$

The Jet Clustering algorithm, with the cone radius set to 0.7 , was chosen to identify jets for this analysis. Two types of jets are defined:

- Calorimeter Jets - These are jets reconstructed using the energy deposited in the calorimeter towers.

- Hadron Jets - These are jets reconstructed using stable hadrons found 
via Monte Carlo truth information.

The hadron jets represent the Monte Carlo truth information and are used to make theoretical predictions for the jet cross section. Since the energy of stable hadrons is detected in the calorimeters the energy of calorimeter and hadron jets should be equal to one another. In this chapter the Jet Clustering algorithm is described and energy corrections that are required to compare hadron jets to calorimeter jets are discussed.

\subsection{The Jet Clustering Algorithm}

Four-vectors for the calorimeter towers are used as the input to the algorithm[1. For Calorimeter jets, the four-vectors are defined using the energy deposited within the towers and the position of the primary vertex.

To reconstruct hadron jets in Monte Carlo simulation the four-vectors of calorimeter towers can be defined using the stable hadrons generated. The $\phi$ and $\eta$ of the hadrons are used to define $\phi$ and $\eta$ for the electromagnetic and hadronic components of the tower. 60\% (40\%) of the hadron energy is assigned as electromagnetic (hadronic) energy. Defining the towers using this simplified technique allow the kinematics to be defined using the same formulae as for calorimeter towers. These can then be used as the input to the jet reconstrotction algorithm. The explicit splitting of energy using the ration 60:40 is investigated in appendix B.

Towers with $E_{T}$ greater than $1 \mathrm{GeV}$ are used as trial geometrical centres for the jets. Neighbouring towers (lying within a radius of 0.7 in $\eta$ - $\phi$ space) with $E_{T}$ exceeding $100 \mathrm{MeV}$ are added to the centres. A new central point

\footnotetext{
${ }^{1}$ The equations defining the kinematics can be found in appendix A
} 
(centroid) is calculated using equations 4.2 , where i denotes all towers contained within the cone, $\mathrm{C}$, under consideration. If $\eta^{C}$ and $\phi^{C}$ are equal to $\eta$ and $\phi$ of the trial tower, the cone is said to be "stable" and is retained, otherwise the new centroid is used to make a new trial cone. Towers within a radius of 0.7 of the new cone are added. All towers that were added previously are kept even if they now lie outside the radius. The centroid is recalculated and the stability of the jet checked; this iteration process continues iteratively until a stable cone is found.

$$
\begin{array}{r}
\eta^{C}=\frac{\sum_{i \subset C} E_{T}^{i} \eta^{i}}{E_{T}^{C}} \\
\phi^{C}=\frac{\sum_{i \subset C} E_{T}^{i} \phi^{i}}{E_{T}^{C}} \\
E_{T}^{C}=\sum_{i \subset C} E_{T}^{i}
\end{array}
$$

In this algorithm, towers can be assigned to more than one jet, resulting in overlapping jets. If the shared energy between the two overlapping jets is greater than $75 \%$ the two jets are merged; otherwise the jets are split, with the shared towers being assigned to the cone that is closest in $\eta-\phi$. Finally the four-vectors of the jets are calculated by summing the four-vectors of the towers within them. The efficiency of the algorithm is greater than $99 \%$ for jets above $15 \mathrm{GeV}$ [40]. 


\subsection{Generic Jet Energy Corrections}

Before any comparisons can be made between calorimeter jets and hadron jets a variety of corrections need to be made to account for effects such as variations in detector response over $\eta$, non linearities in energy response and energy loss due to un-instrumented regions in the detector, as well as the effects of multiple interactions in the events. Detailed studies have been carried out by the CDF collaboration in order to define a standard set of energy corrections that can be applied to all jets 41] together with an associated systematic uncertainty [42]. The corrections applied to jets in the analysis are briefly described in this section.

\subsubsection{Relative Jet Energy Correction}

This correction is applied to account for the variations in detector response with $\eta$. A process know as "Di-jet balancing" is used to find this correction; the transverse energy of the two jets in a $2 \rightarrow 2$ process should be equal regardless of the jet $\eta$. The transverse energy of jets inside the region $0.2<$ $|\eta|<0.6$ is compared to the transverse energy of jets outside, and the ratio is taken as a correction factor. The factor varies as a function of $\eta$ and ranges between 0.85 and 1.05 [41. A systematic uncertainty of $2 \%$ [42] is used for the correction to account for uncertainties in the procedure, remaining fluctuations and time dependence. 


\subsubsection{Absolute Jet Energy Correction}

Absolute corrections are applied to account for any non linearity in energy response and energy loss in un-instrumented regions of each calorimeter. The correction depends on the calorimeter response to neutral and charged pions. These were determined during Run I for jets generated using a tuned Monte Carlo, and the correction found by comparing the $\sum p_{t}$ of tracks to the calorimeter energy deposited. The correction factor falls as a function of $E_{t}$, a $40 \mathrm{GeV}$ jet is corrected by a factor of 1.25 [41]. The systematic error assigned to the correction is 3.0-2.5\% [42] depending on jet $E_{t}$.

\subsubsection{Energy Scale Corrections}

The absolute energy corrections used were measured during Run I. A scale factor is introduced to account for changes in the jet energy scale between Run II and Run I. The energy scale of the central electromagnetic and hadronic calorimeters is set and events containing high energy photons balanced by a recoiling jet are used to find the Run I - Run II scale factor.

The energy scale of the Central Electromagnetic Calorimeter is set by reconstructing the $\mathrm{Z}$ mass peak. The conversion factors used to convert the raw photomultiplier data to energy measurements in $\mathrm{GeV}$ are adjusted such that the $\mathrm{Z}$ mass is reconstructed to be $91.05 \pm 0.29 \mathrm{GeV}$. The energy scale of the Central Hadronic Calorimeter is set by comparing the Run I and Run II peak for energy deposits by muons (minimum ionising particles) in the detector and taking any difference as a correction. The difference in the ratio of photon energy to jet energy between Run I and Run II is used as the energy scale correction. For 0.7 cones this is 1.042 [41], this implies that 
jet energies in Run II are $4.2 \%$ lower than in Run I, since the source of the discrepancy is not well understood a $100 \%$ [42] systematic uncertainty is applied to this correction.

\subsubsection{Multiple Interaction Correction}

Energy deposited by particles in overlapping minimum bias events may fall into the jet clustering cone, and so needs to be subtracted. Using minimum bias events a cone of radius 0.7 is constructed using a random $\eta-\phi$ position, the transverse energy of the cones are parametrised as a function of the number of vertices in the event. The contribution from multiple interactions is found to be $0.781 \mathrm{GeV} /$ vertex [41] and is used as the multiple interaction correction. The correction is assigned a systematic uncertainty of 100 $\mathrm{MeV} /$ vertex [42].

\subsection{Correction for Jets Originating From b Quarks}

A Pythia Monte Carlo sample simulating $2 \rightarrow 2$ processes, with the minimum $p_{t}$ of the hard scatter set to $18 \mathrm{GeV}$, is used to investigate how well the energy of calorimeter jets matches the energy of hadron jets. Hadron and calorimeter jets are constructed, and the calorimeter jets corrected using the generic corrections described in section 4.2. For jets within the central region, $|\eta|<1.2$, hadron jets are matched to calorimeter jets by requiring the distance between the two in $\eta$ - $\phi$ space, $\Delta H^{2}$, to be less than 0.2 . Figure

$$
{ }^{2} \Delta R=\sqrt{\Delta \eta^{2}+\Delta \phi^{2}}
$$


4.1 shows the hadron jet $E_{t}$ divided by the corrected calorimeter jet $E_{t}$, as a function of hadron jet $E_{t}$ for all jets. The fit starts at $25 \mathrm{GeV}$ in order to avoid any biasing from minimum $p_{t}$ requirements of the hard scatter. It can be seen that after jet corrections, on average, the jet $E_{t}$ is underestimated by a factor of $7 \%$.

The jets are split into various categories in order to understand the source of the discrepancy. Hadron jets are classified as b jets if a b hadron is found within a cone of 0.7 around the jet axis $(\Delta R(j e t-B)<0.7)$. If this criteria is not satisfied and a c hadron is found within a cone of 0.7 the jet is classified as a $\mathrm{c}$ jet. If the jet is neither a b jet or $\mathrm{c}$ jet but does contain a gluon then it is classified as a gluon jet. All remaining jets fall into the uds jets category, having originated from a up, down or strange quark. The ratio of hadron jet $E_{t}$ to corrected calorimeter jet $E_{t}$ for the different categories is shown in figure 4.2. It can be seen that for uds jets the corrections do correct the calorimeter jet $E_{t}$ to the original hadron jet $E_{t}$; however for all other types of jet the corrections underestimate the $E_{t}$; by $6.8 \%$ for gluon jets, $9.8 \%$ for c jets, and $16 \%$ for b jets.

Each of the jet corrections is adjusted by $\pm 1 \sigma$ in order to see if the discrepancy is covered by the systematic errors calculated for the jet corrections. Figures 4.3 to 4.6 show the ratio of hadron jet $E_{t}$ to calorimeter jet $E_{t}$ when each correction is modified by $\pm 1 \sigma$. The systematics cover the discrepancy for gluon jets and c jets, as the energy ratio shifts between 1.0 and 1.1 when the one sigma systematics are applied. However the discrepancy for b jets is still significantly higher than this and so a further correction is necessary.

The semi-leptonic decay of b hadrons, and c hadrons further down the de- 


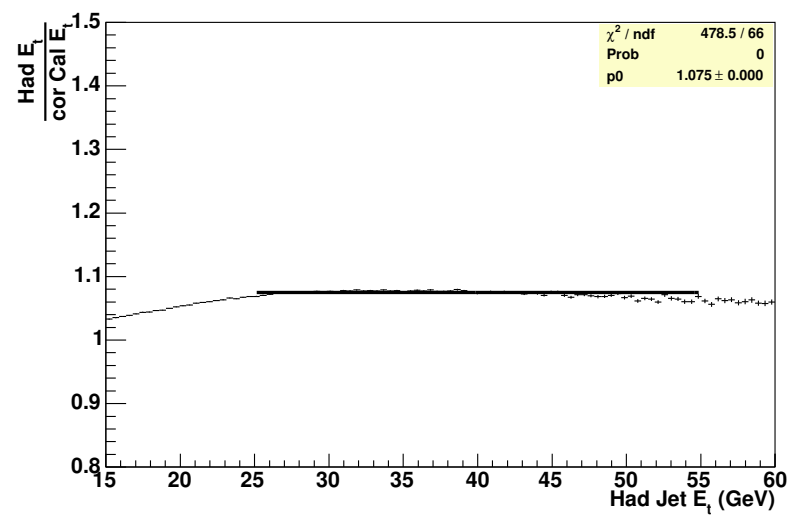

Figure 4.1: Plot showing the mean hadron (Had) jet $E_{t}$ divided by corrected calorimeter (cor Cal) jet $E_{t}$ per bin, as a function of the hadron jet $E_{t}$ (profile plot). The solid line represents a fit to a constant function, the fit parameters are shown in the top right corner.

cay chain is the main reason b jets will have a lower energy in the calorimeter. The decay produces neutrinos resulting in missing energy within the b jets; when the hadron decay involves muons additional energy is also lost as the muon will deposit very little energy in the calorimeters. Additional energy is also lost when taus are involved due to the decay of the tau. The ratio of hadron jet $E_{t}$ to calorimeter jet $E_{t}$ for non semi-leptonic decays and different categories of semi-leptonic decays is shown in figure 4.7. As expected the ratio is smallest for non semi-leptonic decays, larger for decay to an electron and larger still for decay to muons and taus.

The correction for $\mathrm{b}$ jets is defined as the ratio of hadron jet $E_{t}$ to calorimeter jet $E_{t}$. The profile plot for this ratio (figure 4.2(a) is fitted using a constant function and gives a value for the correction of 1.157. Figure 4.8 shows that applying the correction brings the ratio of calorimetric 


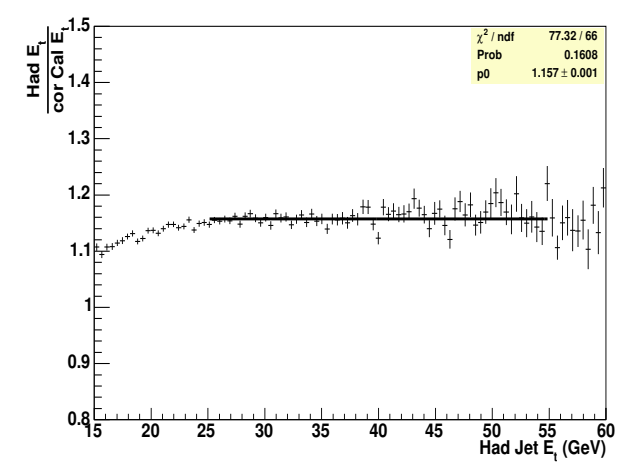

(a) b jets

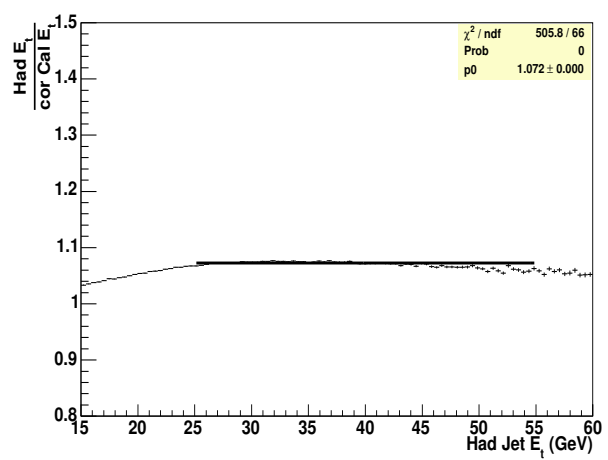

(c) gluon jets

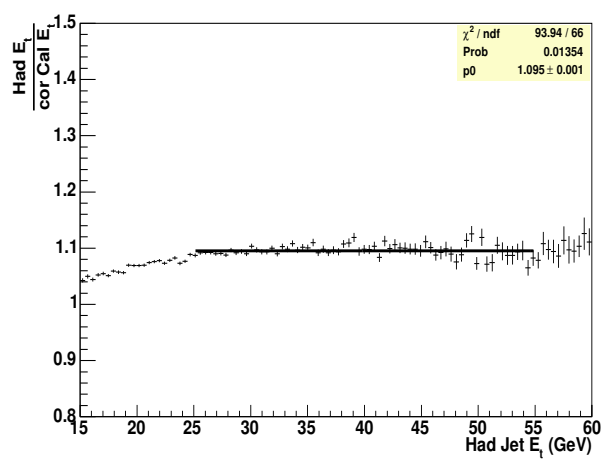

(b) c jets

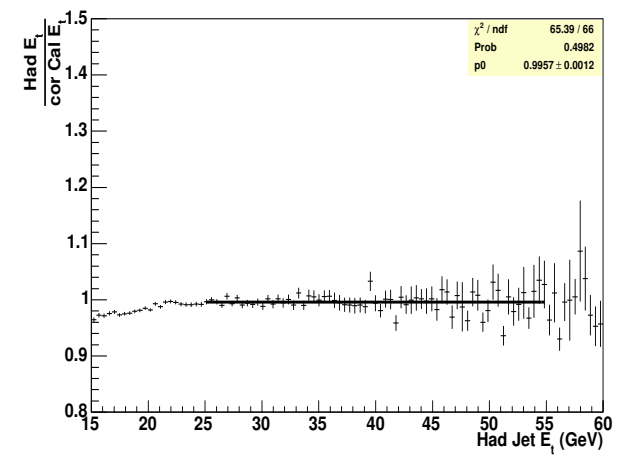

(d) uds jets

Figure 4.2: Profile plots showing hadron (Had) jet $E_{t}$ divided by corrected calorimeter (cor Cal) jet $E_{t}$, as a function of the hadron jet $E_{t}$, for: $\left.a\right) b$ jets; b) c jets; c) gluon jets; d) uds jets. The solid line represents a fit to a constant function, the fit parameters are shown in the top right corner of each plot. 

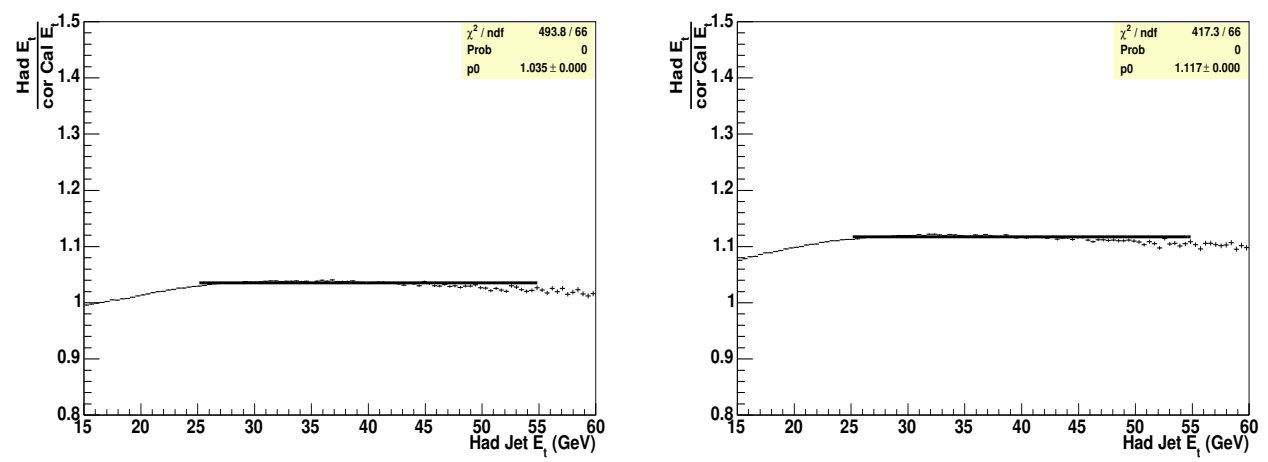

Figure 4.3: Profile plots showing the ratio of hadron (Had) jet $E_{t}$ to corrected calorimeter (cor Cal) jet $E_{t}$, for gluon- and uds jets combined, when the relative energy correction is adjusted by $+1 \sigma$ (left) and $-1 \sigma$ (right). The solid line represents a fit to a constant function, the fit parameters are shown in the top right corner.
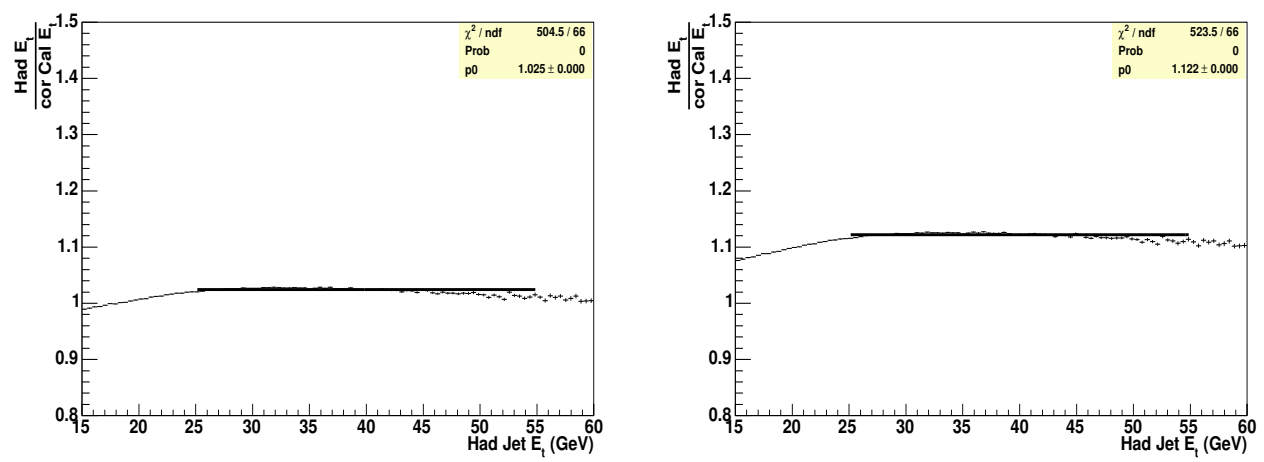

Figure 4.4: Profile plots showing the ratio of hadron (Had) jet $E_{t}$ to corrected calorimeter (cor Cal) jet $E_{t}$, for gluon- and uds jets combined, when the jet energy scale correction is adjusted by $+1 \sigma$ (left) and $-1 \sigma$ (right). The solid line represents a fit to a constant function, the fit parameters are shown in the top right corner. 

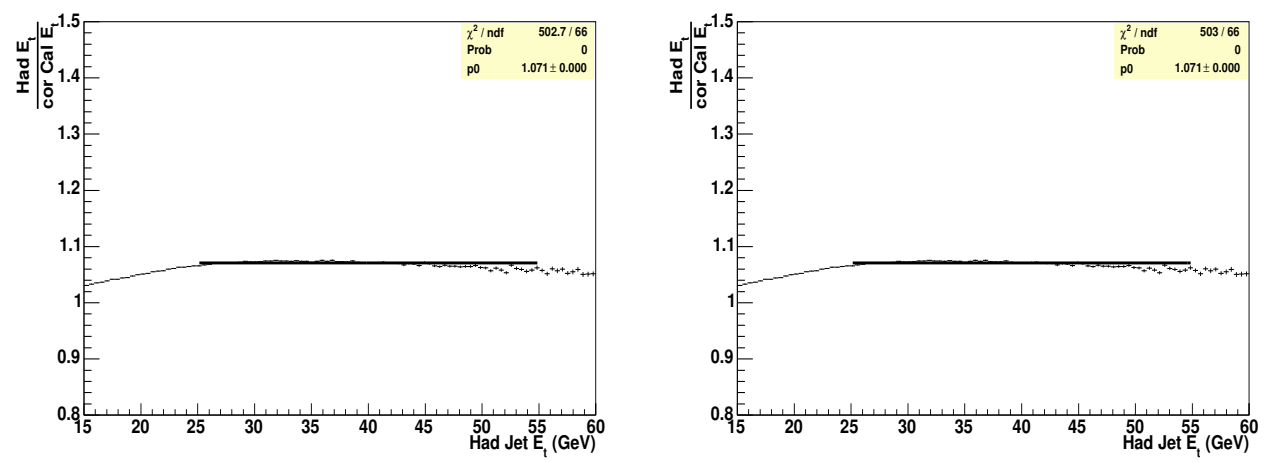

Figure 4.5: Profile plots showing the ratio of hadron (Had) jet $E_{t}$ to corrected calorimeter (cor Cal) jet $E_{t}$, for gluon- and uds jets combined, when the multiple interaction correction is adjusted by $+1 \sigma$ (left) and $-1 \sigma$ (right). The solid line represents a fit to a constant function, the fit parameters are shown in the top right corner.
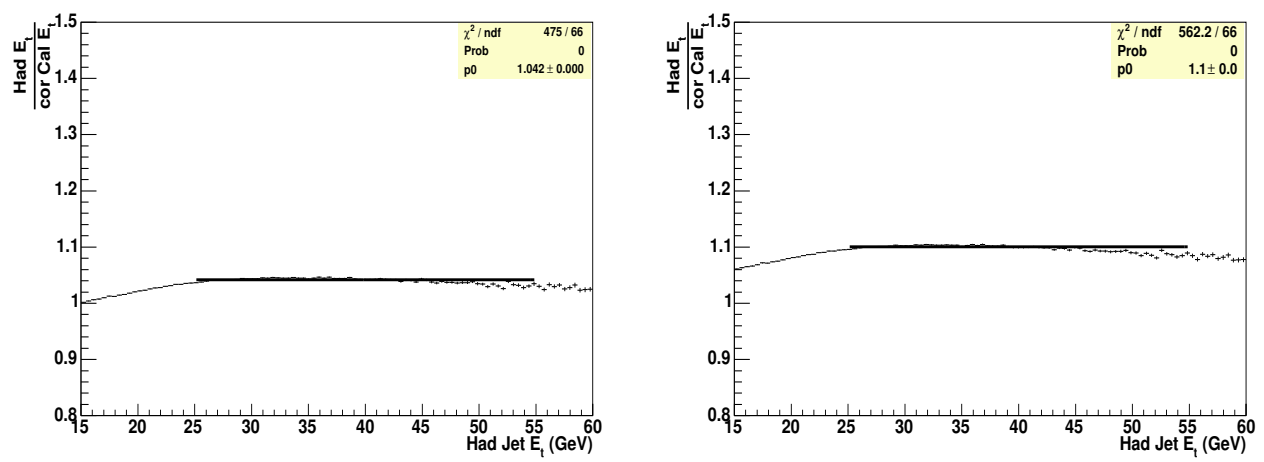

Figure 4.6: Profile plots showing the ratio of hadron (Had) jet $E_{t}$ to corrected calorimeter (cor Cal) jet $E_{t}$, for gluon- and uds jets combined, when the absolute energy correction is adjusted by $+1 \sigma$ (left) and $-1 \sigma$ (right). The solid line represents a fit to a constant function, the fit parameters are shown in the top right corner. 


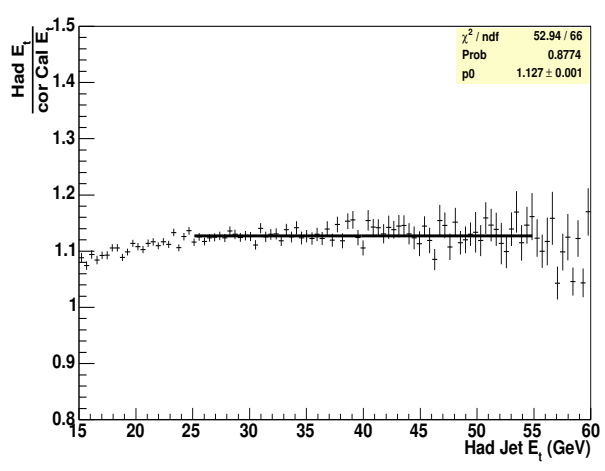

(a) non semi-leptonic decays

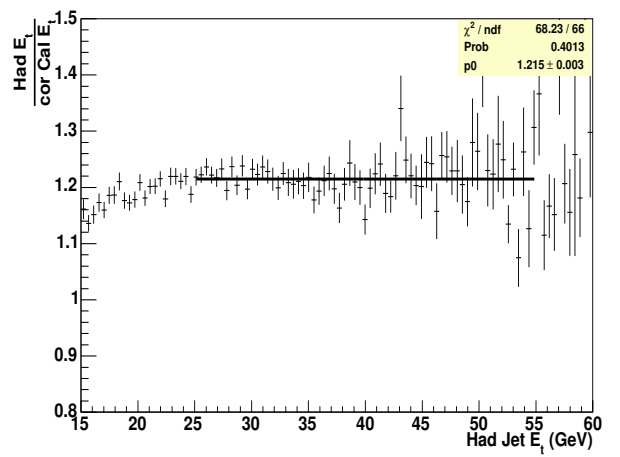

(c) muon in decay chain

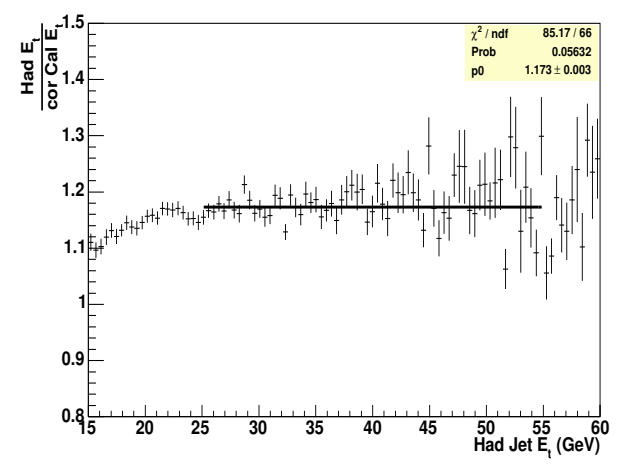

(b) electron in decay chain

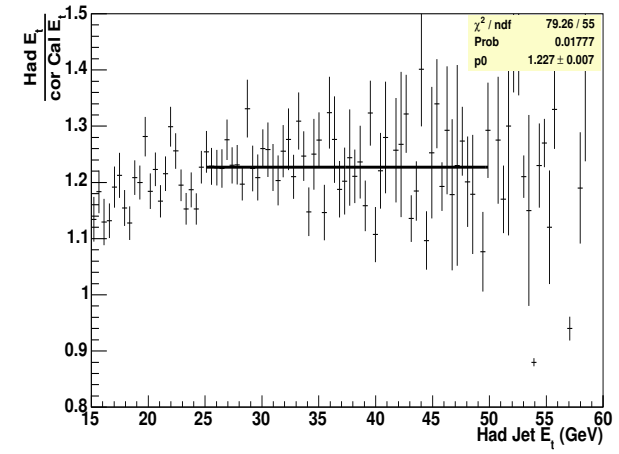

(d) tau in decay chain

Figure 4.7: Profile plots showing hadron (Had) jet $E_{t}$ divided by corrected calorimeter (cor Cal) jet $E_{t}$, as a function of the hadron jet $E_{t}$, for: a) non semi-leptonic $b$ decay; b) b decay involving an electron; c) b decay involving $a$ muon; d) b decay involving a tau. The solid line represents a fit to a constant function, the fit parameters are shown in the top right corner. 
to hadronic energy back to 1 . The systematic uncertainty for the b jet correction is determined by repeating the study using a Herwig Monte Carlo sample. The corresponding plots for Herwig can be found in Appendix C, and show that Herwig exhibits the same behaviour. The ratio of hadron jet $E_{t}$ to calorimeter $E_{t}$ is found to be 1.172 , the difference between this and the Pythia value is taken as an estimate for the systematic uncertainty for the $b$ jet correction.

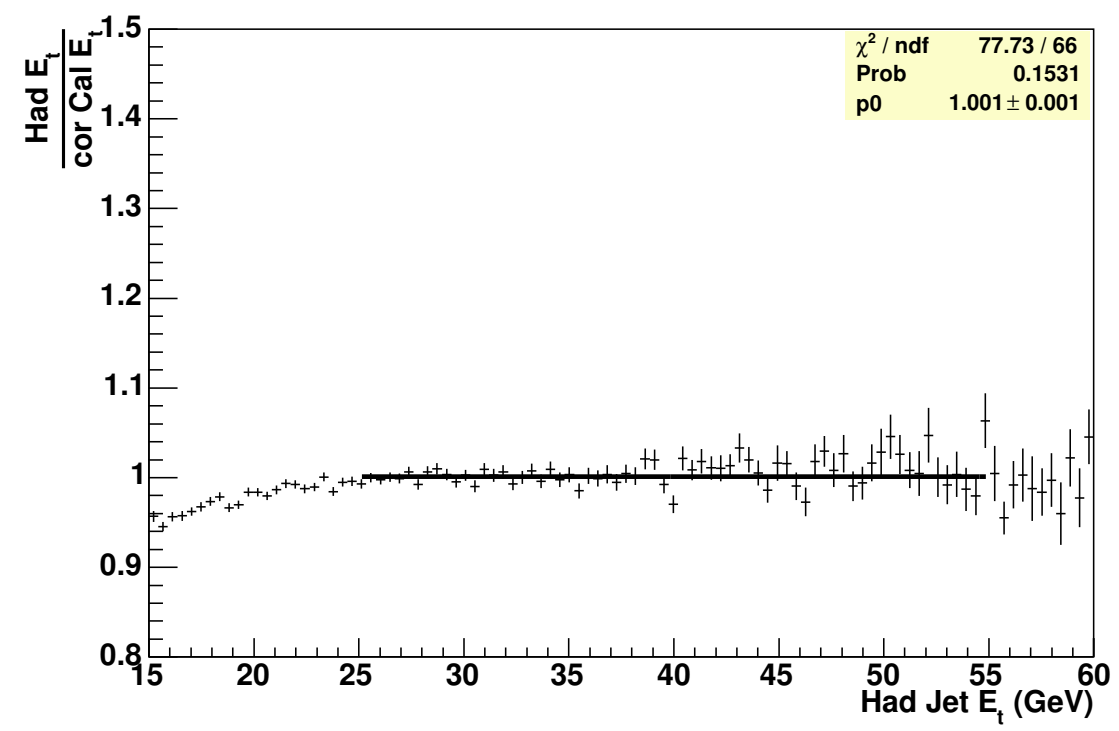

Figure 4.8: Profile plot showing hadron (Had) jet $E_{t}$ divided by corrected calorimeter (cor Cal) jet $E_{t}$ for b jets as a function of the hadron jet $E_{t}$ after applying the $b$ jet correction to them. The solid line represents a fit to a constant function, the fit parameters are shown in the top right corner. 


\subsection{Use for Cross Section Analysis}

The jet clustering algorithm described in section 4.1 is used to reconstruct jets in the data sample used for the cross section analysis. The energy of the jets is corrected using the generic corrections described in section 4.2 and then multiplied by the $\mathrm{b}$ jet correction described in section 4.3 . The final cross section is then quoted with respect to this fully corrected energy. 


\section{Chapter 5}

\section{Secondary Vertices}

The lifetime of the b quark results in b hadrons travelling a short distance before decaying. Precision measurements of charged particle position in the silicon detectors allows the decay ("secondary") vertex of the b hadron, which is typically separated from the primary interaction vertex by $\sim 1 \mathrm{~mm}$, to be identified. Figure 5.1 illustrates how tracks from the decay of a particle that has travelled a projected distance $L_{x y}$ (Decay Length) can be identified due to their large impact parameter $\left(d_{0}\right)$. These tracks can be used to reconstruct the secondary vertex and is the basis for the CDF secondary vertex tagging algorithm, "SECVTX". This chapter describes how tracks are reconstructed and used by SECVTX to tag jets originating from heavy flavour decay.

The tagging algorithm is used to identify b jets for the cross section calculation, hence the efficiency of the tagging algorithm needs to be measured. A novel technique used to measure the efficiency of the tagging algorithm in data is described in this chapter. Section 5.3 explains how the purity of a data sample can be measured using distributions from Monte Carlo and section 5.4 goes on to describe how the measured purity is used to find the 
efficiency in an electron triggered dataset.

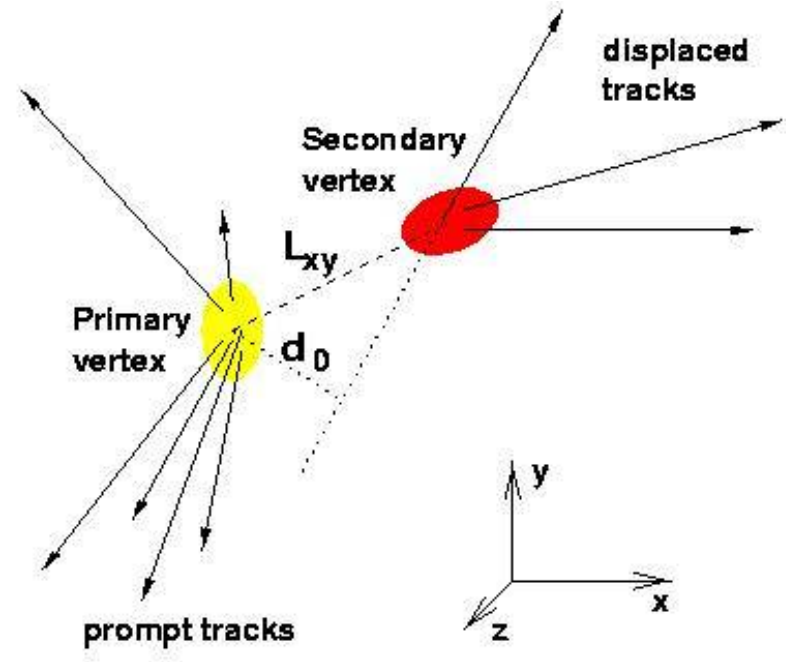

Figure 5.1: Schematic diagram showing how a long-lived particle produced at the primary vertex will travel a distance $L_{x y}$ in the $r-\phi$ plane before decaying at the secondary vertex. The tracks from the decay have a large distance of closest approach $\left(d_{0}\right)$ to the primary vertex.

\subsection{Track Reconstruction}

The magnetic field in CDF points in the negative z direction. Charged particles move in a helical trajectory where the axis of the helix is parallel to the $\mathrm{z}$ axis, and the projection of the track onto the $\mathrm{x}-\mathrm{y}$ plane is circular. The five parameters used to fully define the helix are:

- $\cot \theta$ - the cotangent of the polar angle at minimum approach to the origin of the helix. 
- $C$ - half the radius of curvature of the track (same sign as particle charge).

- $z_{0}-\mathrm{z}$ position at point of minimum approach to the origin of the helix.

- $d_{0}$ - impact parameter, distance between origin and helix at point of minimum approach to the origin.

- $\phi_{0}$ - Azimuthal angle at point of minimum approach to the origin.

The sign of the impact parameter is found using equation 5.1, where $\hat{z}$ is a unit vector in the $\mathrm{z}$ direction, $\vec{r}$ is a vector pointing from the primary vertex to the point of minimum approach and $\overrightarrow{p_{t}}$ is the transverse momentum vector.

$$
d_{0}=\frac{\hat{z} \cdot \vec{r} \times \overrightarrow{p_{t}}}{\left|\overrightarrow{p_{t}}\right|}
$$

Tracks are fully reconstructed using COT hit information [43]. The reconstruction algorithm begins by combining hits in each superlayer to make "segments". Track finding proceeds initially by looking for tracks in the r- $\phi$ plane using two algorithms called "Segment Linking" and "Histogram Linking", which run in parallel. The segment linking algorithm makes tracks by linking segments found in the axial superlayers, starting from the outer superlayers and working inwards. Histogram linking uses the beam position and position of a segment to define a reference radius for a circle. All hits corresponding to the track should lie close to this reference radius. For each superlayer the distance of the hits from the reference radius is histogrammed and the histograms from each superlayer are summed. The hits along the track will lie in the same bin of the histogram and thus identify the track. A 
three parameter fit is performed on the identified track to determine $C, \phi_{0}$ and $d_{0}$.

Tracks made using both axial algorithms are then linked to segments in the stereo superlayers using a linear fit in the r-z plane. After all tracks have been found a final track fit is performed using all five helical track parameters. Since tracks from both algorithms are used, a search is performed to remove any duplicate tracks. The efficiency of the algorithm has been measured to be $99.61 \%$ for finding non-isolated muon tracks with a $p_{t}>1.5 \mathrm{GeV}$ [44].

Silicon information is used only after COT tracks have been made. The tracking algorithm starts from the outermost silicon layer and works inwards (OI algorithm) [45]. Tracks from the COT are extrapolated inwards to the silicon. For each silicon layer, the error matrix of the track is used to find any axial hits that are within four standard deviations of the track circle parameters. Whenever hits are added to the track, it is refitted and a new error matrix calculated. Tracks are kept if they have at least three silicon hits. The OI algorithm has a track finding efficiency of $95.2 \%$ for tracks with $p_{t}>1.5 \mathrm{GeV}[45]$.

\subsection{SECVTX}

The secondary vertex tagging algorithm was originally developed in Run I to identify b jets from top quark decays [46] and seeks displaced vertices in the $\mathrm{x}-\mathrm{y}$ plane as evidence of heavy quark decay. It begins by looping over the subset of the reconstructed tracks in each event that meet a variety of COT and silicon hit requirements. All jets with a transverse energy greater than $15 \mathrm{GeV}$ are considered for tagging. Selected tracks that lie within a 
cone of radius 0.7 of the jet axis are associated to each jet. Displaced tracks are selected by requiring the track impact parameter significance, given by equation 5.2, to be greater than 2.5. Using a vertex fitting routine [47] two displaced tracks are used to make a seed vertex, a successful vertex fit requires a $\chi^{2}$ less than 50 . The impact parameter significance with respect to the seed vertex is calculated for the other displaced tracks within the jet and those with a value less than 3 are added to the vertex. If no tracks are added the process is repeated with a new seed vertex made up of another two displaced tracks. The iteration continues until a secondary vertex with a minimum of three tracks is found or all track combinations have been attempted. If a secondary vertex is not found using this method then a second method is employed. A vertex fit is performed using all tracks with $S_{d_{0}}$ greater than 3.0. If any tracks have a $\chi^{2}$ greater than 1000 , the track with the largest $\chi^{2}$ is removed and the vertex is refitted, in an iterative process which continues until all tracks used in the fit have a $\chi^{2}$ less than 1000. If a vertex is found using either method a veto is placed on long-lived Kaons by requiring the difference in the invariant mass of the tracks associated with the vertex and the $K^{0}$ mass to be greater than $0.02 \mathrm{GeV}$. The jet is tagged if the following requirements are met:

- $\chi^{2}($ Vertex Fit $)<2000$

- $\left|L_{x y}\right|<5.0 \mathrm{~cm}$

- $L_{x y} / \sigma_{L_{x y}}>3.0$

$$
S_{d_{0}}=\left|d_{0}\right| / \sigma_{d_{0}}
$$




\subsection{Purity of Heavy Flavour Samples}

The distributions of the secondary vertex mass of tagged jets and momentum relative to the jet axis of any leptons in the decay are different for b, c and light jets. It is therefore possible to fit the total distribution for a sample of events to extract the relative fractions, where the individual distributions are taken from Monte Carlo. The distributions taken from Monte Carlo are defined as templates.

\subsubsection{Lepton $p_{t}$}

Due to the greater mass of $b$ hadrons, leptons coming from these decays have a greater transverse momentum relative to the jet direction $\left(p_{t}^{r e l}\right)$, compared to those from c hadron decay and light hadrons? Figure 5.2 shows the respective flavour distributions. A clear discrimination is seen between b jets and $\mathrm{c}$ jets, however the discrimination between light jets and $\mathrm{c}$ jets is not as good.

Figure 5.3 shows the $p_{t}^{\text {rel }}$ variable is dependent on jet $E_{t}$. Dividing the $p_{t}^{\text {rel }}$ by the $E_{t}$ of the jet provides a variable that largely removes the dependence on the jet $E_{\mathrm{t}}^{2}$. Figure 5.4 shows there is still some residual variation for $\mathrm{b}$ jets. The $\mathrm{b}$ jet templates corresponding to each jet $E_{t}$ bin are used when measuring the efficiency as a function of jet $E_{t}$ to account for this variation.

\footnotetext{
${ }^{1}$ The most common source for obtaining leptons from light hadrons is through photon conversions in the beam pipe or inner silicon layers.

${ }^{2}$ From here on in the use of $p_{t}^{r e l}$ implies $p_{t}^{r e l} /$ jet $E_{t}$
} 


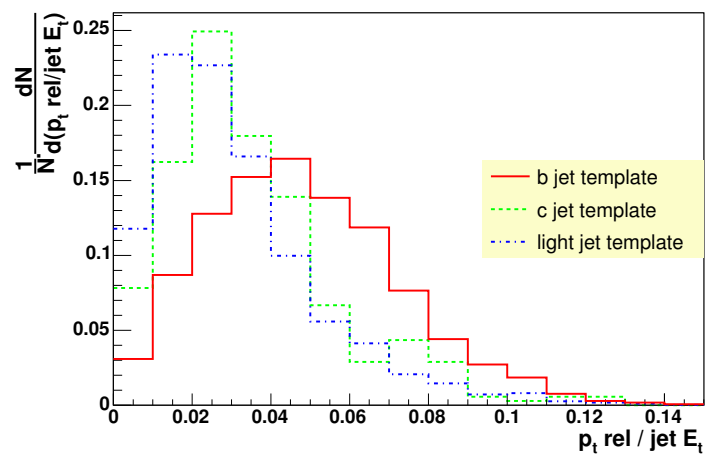

Figure 5.2: Distributions for $p_{t}^{\text {rel }} /$ jet $E_{t}$ for electrons within $b$ jets, $c$ jets and light jets.

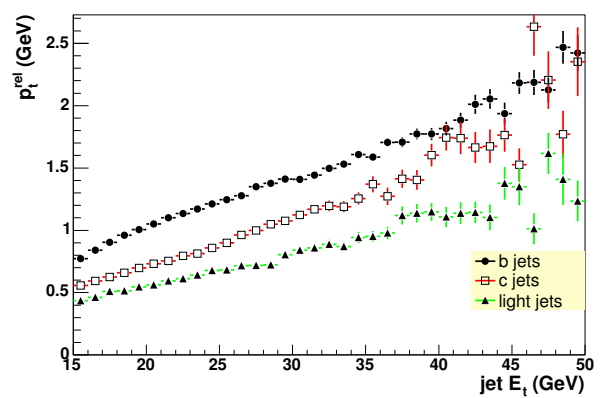

(a) $p_{t}^{r e l} \mathrm{v}$ jet $E_{t}$

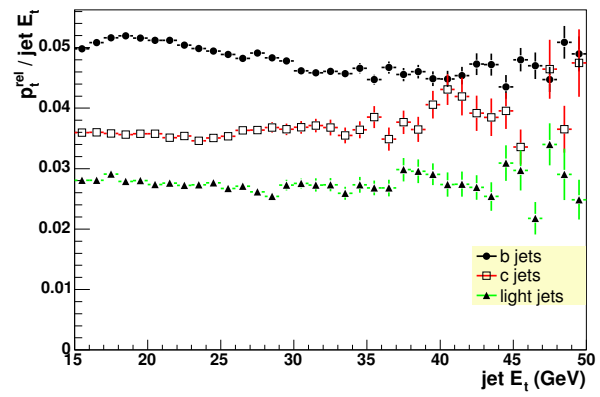

(b) $\frac{p_{t}^{r e l}}{j e t E_{t}} \mathrm{v}$ jet $E_{t}$

Figure 5.3: Profile plot showing the dependence on jet $E_{t}$ of: a) $p_{t}^{\text {rel }}$; b) $p_{t}^{r e l} /$ jet $E_{t}$. 

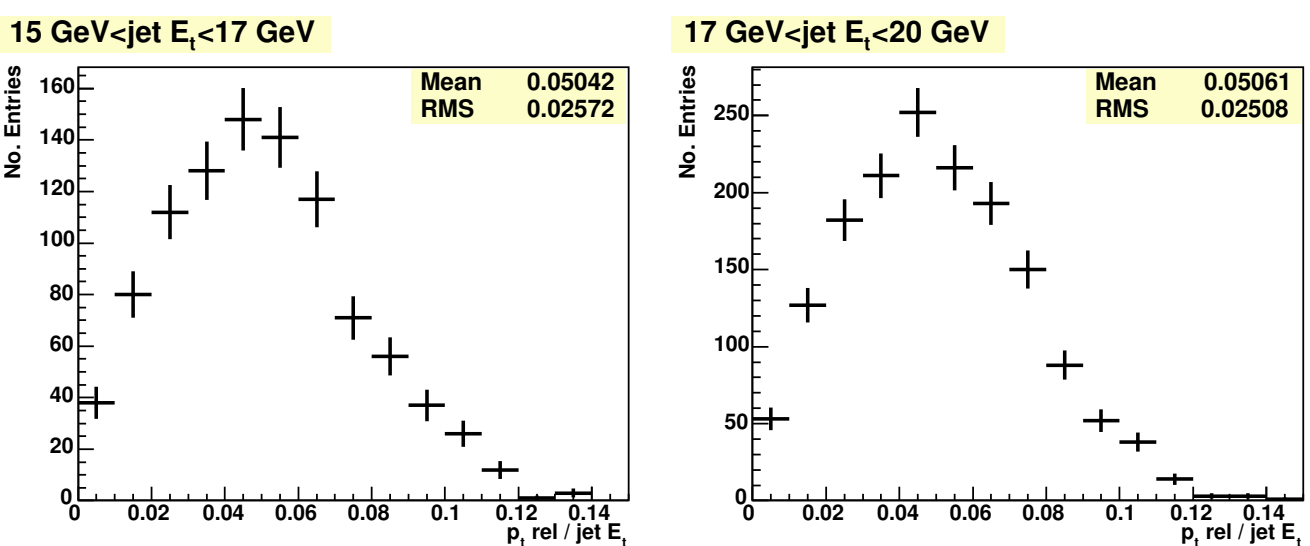

$20 \mathrm{GeV}_{<j e t} \mathrm{E}_{\mathrm{t}}<24 \mathrm{GeV}$

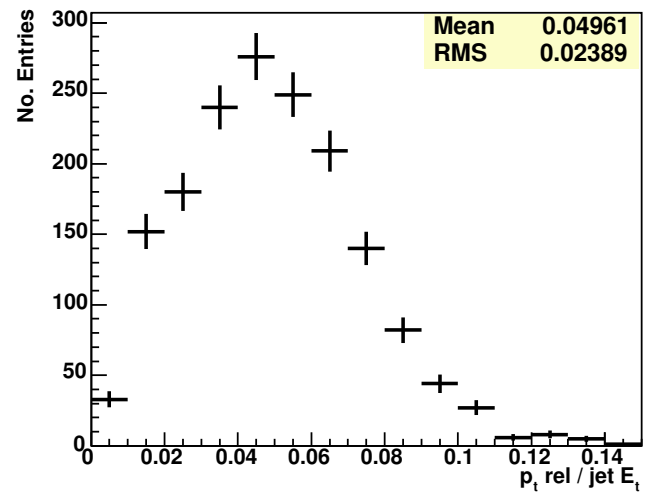

\section{$24 \mathrm{GeV}<j e t \mathrm{E}_{\mathrm{t}}<28 \mathrm{GeV}$}

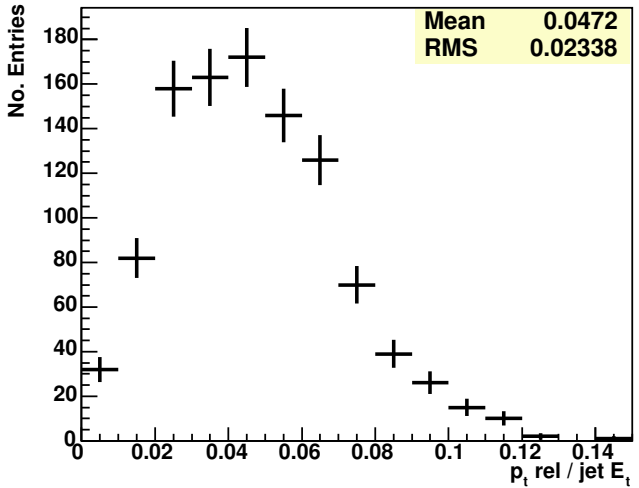

$28 \mathrm{GeV}_{<j e t} \mathrm{E}_{\mathrm{t}}<40 \mathrm{GeV}$

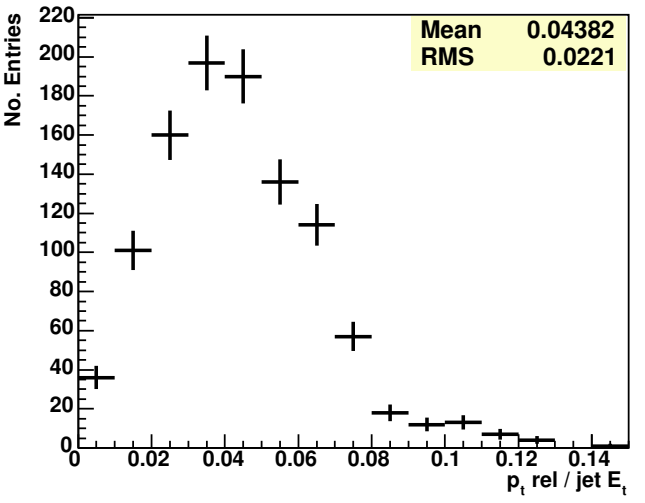

jet $E_{t}>40 \mathrm{GeV}$

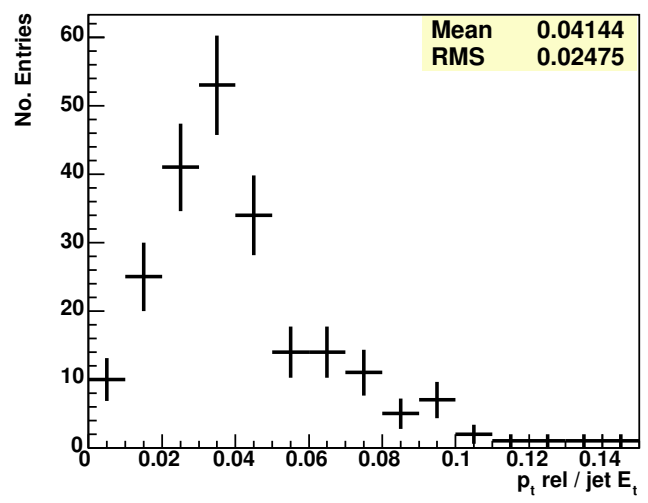

Figure 5.4: $p_{t}^{\text {rel }} /$ jet $E_{t}$ spectrum for $b$ jets for various jet energy ranges. 


\subsubsection{Secondary Vertex Mass}

The invariant mass spectrum of the tracks associated with secondary vertices $3^{3}$ found using SECVTX is shown in figure 5.5 for b, c and light jets. It shows a clear discrimination between $\mathrm{b}$ jets and c jets, and to a lesser extent, between $c$ jets and light jets. $b$ jet secondary vertex masses in particular extend above $2 \mathrm{GeV}$ which is rarely the case for c jets or uds jets. Figure 5.6 shows the secondary vertex mass has very little dependence on jet $E_{t}$, which allows a single high statistics template to be used for all jet $E_{t}$ bins.

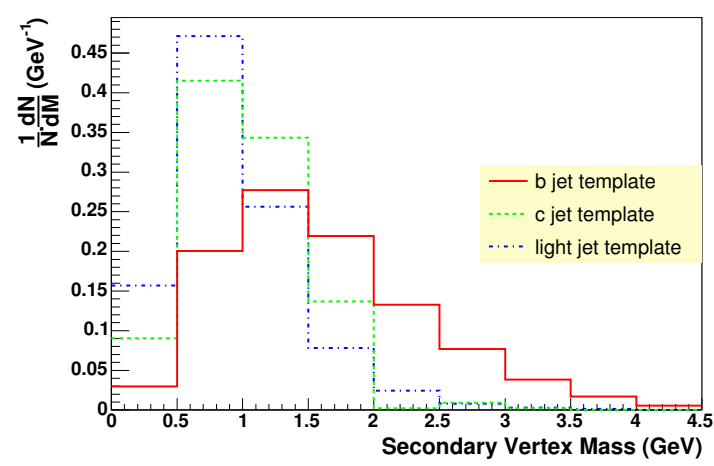

Figure 5.5: Invariant mass of tracks associated with secondary vertices found within $b$ jets, c jets and light jets.

\subsubsection{Fitting the $b$ fraction}

Using templates for $\mathrm{b}$ and non $\mathrm{b}$ jets, the fractions of each in a data sample can be measured using a ROOT fitting function [48] called TFractionFitter [49]. This performs a bin by bin fit to the data taking into account the statistical uncertainties in bin contents in both the data and the templates.

\footnotetext{
${ }^{3}$ When calculating the invariant mass it is assumed that all tracks have pion mass.
} 


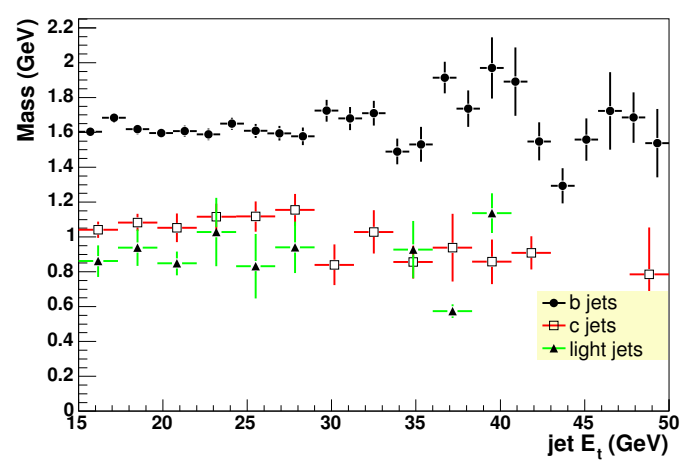

Figure 5.6: Profile plot showing the secondary vertex mass as a function of jet $E_{t}$ for $b, c$ and light jets.

The routine returns the fraction that each template contributes to the total histogram, the covariance matrix and the chi-squared of the fit. Also returned is a predicted histogram which is an addition of the modified templates scaled by the calculated fractions.

\subsection{SECVTX Efficiency Measurement}

Data samples containing high $p_{t}$ electrons are used to measure the tagging efficiency since the presence of a lepton enriches the b quark content and allows the purity of the sample to be measured. Having found the $\mathrm{b}$ fraction of a sample of jets before and after applying SECVTX the tagging efficiency can be found using equation 5.3. $N^{j e t}$ is the total number of jets and $N^{t a g}$ is the number of tagged jets, both of which are taken from data. $F_{b}^{j e t}$ and $F_{b}^{\text {tag }}$ are the $\mathrm{b}$ fractions before and after applying SECVTX and are taken from 
Monte Carlo fits to the data, as described in section 5.3.3.

$$
\epsilon_{b}=\frac{F_{b}^{\text {tag }} N^{t a g}}{F_{b}^{j e t} N^{j e t}}
$$

\subsubsection{Data Samples and Event Selection}

For the data an $8 \mathrm{GeV}$ inclusive electron triggered sample is used. The trigger path requires an $8 \mathrm{GeV}$ electromagnetic object with an $8 \mathrm{GeV}$ track linked to it at level 1 . For level 2, in addition to the level 1 requirements, a $2 \mathrm{GeV}$ energy deposit is required in the Shower Maximum detector. For level 3, a reconstructed $8 \mathrm{GeV}$ electron is required.

Herwig and Pythia samples simulating $2 \rightarrow 2$ processes are used to make the template histograms for the purity measurement and make comparisons to data, some of these samples have a Monte Carlo truth level filter, this is known as a HEPG filter, requiring a greater than $7 \mathrm{GeV}$ electron or muon. Figures 5.7 and 5.8 show comparisons between Herwig and Pythia for the $p_{t}^{\text {rel }}$ and secondary vertex mass templates. It can be seen that the shapes of the samples agree so the templates used to fit data are made up of all samples combined.

The electron identification cuts used are derived from the baseline cuts recommended by the CDF Electron Taskforce [50]. The electron identification variables are defined in Appendix D, The cuts used for the electron candidate are taken from an alternative measurement of the tagging efficiency [51] and are listed below:

\footnotetext{
${ }^{4}$ All generated particles and information on their kinematics, decay vertices etc. are stored in the HEPG bank. From here on in all references to HEPG level implies the Monte Carlo truth information has been used.
} 


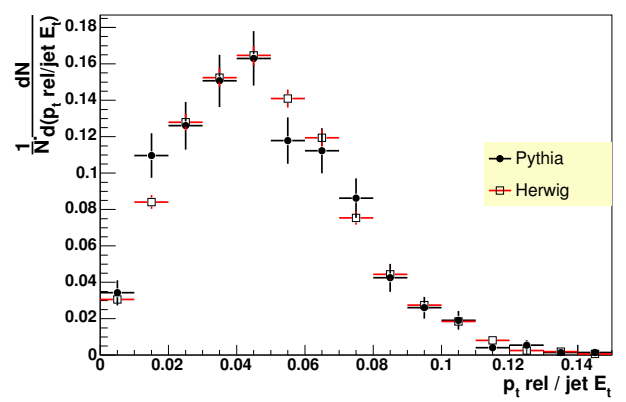

(a) b jets

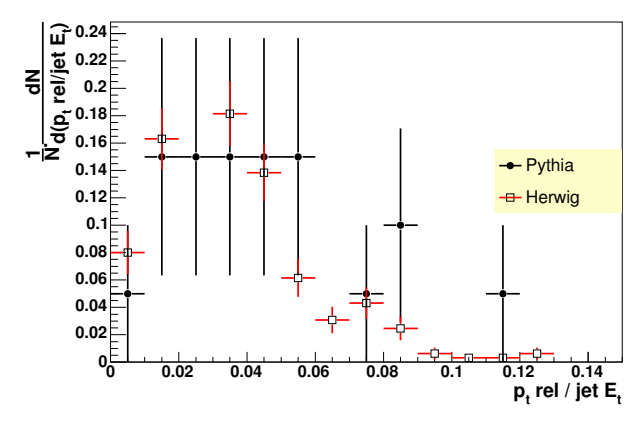

(b) c jets

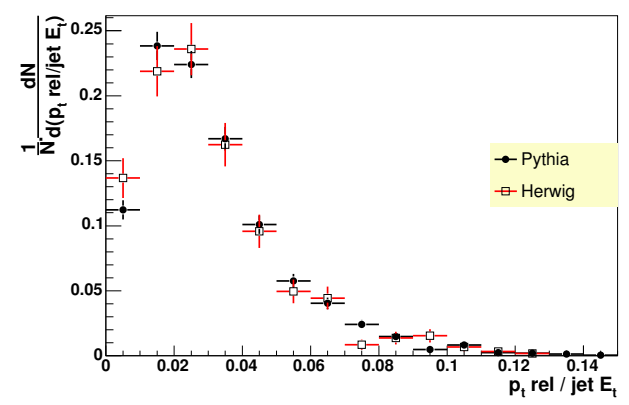

(c) light jets

Figure 5.7: Comparison between Pythia and Herwig for the $p_{t}^{\text {rel }}$ templates for: a) b jets; b) c jets; c)light jets. 


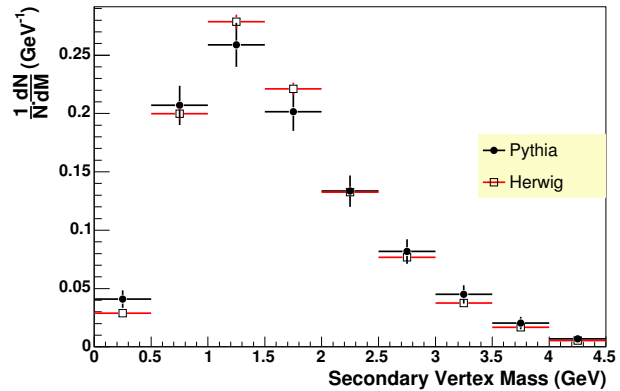

(a) b jets

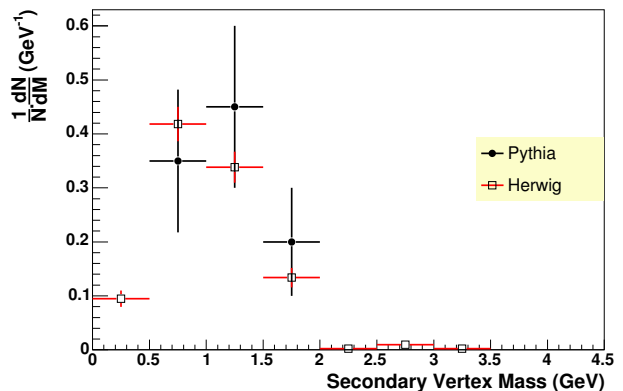

(b) c jets

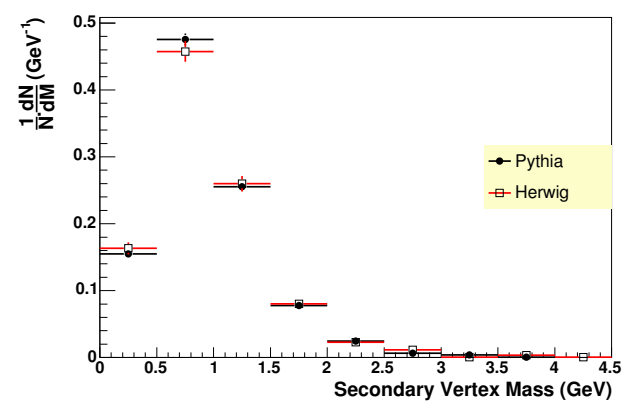

(c) light jets

Figure 5.8: Comparison between Pythia and Herwig for the secondary vertex mass templates for: a) b jets; b) c jets; c)light jets. 
- $E_{t}>9.0 \mathrm{GeV}$

- $p_{t}>4.5 \mathrm{GeV}$

- $0.5<E / P<2.0$

- $\mathrm{Had} / \mathrm{Em}<0.05$

- $\operatorname{Lshr}<0.2$

- CES $|\Delta x|<3 \mathrm{~cm}$

- $\mathrm{CES}|\Delta z|<5 \mathrm{~cm}$

- CES strip $\chi^{2}<10$

- $\left|z_{0}-z_{\text {vertex }}\right|<5 \mathrm{~cm}$

- N Cot Axial Hits for electron candidate $>20$

- N Cot Stereo Hits for electron candidate $>16$

- $\sigma_{d 0}<0.05 \mathrm{~cm}$

In addition to satisfying these criteria, the electron candidate must be in a fiducial region of the electromagnetic calorimeter.

Electron jets are selected by applying a cone jet algorithm with a radius of 0.7 , and requiring jet $E_{t}>15 \mathrm{GeV}$. A $\Delta R$ separation ${ }^{5}<0.7$ between the electron and the jet is required. The electron jet requirement enriches the sample with jets containing b hadrons that have decayed semi-leptonically. The most common source for obtaining electrons from light hadrons is through photon

\footnotetext{
${ }^{5}$ As defined in section 4.3
} 
conversions, to reduce this and further enrich the sample with heavy flavour a second jet tagged with SECVTX is required in the event, which is labelled the "away" jet.

\subsubsection{Efficiency Calculation}

Using the fitting technique described in section 5.3.3, the b flavour content is found before and after applying SECVTX to the electron jets and the efficiency found using equation 5.3. The $p_{t}^{r e l}$ spectrum of the electron jets is used to find the b flavour content $\left(F_{b}^{\text {jets }}\right)$ of the sample before applying SECVTX; the secondary vertex mass spectrum of the tagged electron jets is used to find the content after applying SECVTX $\left(F_{b}^{t a g}\right)$.

Table 5.1 shows the results of the fraction fitting for the Herwig filtered Monte Carlo sample and data, figure 5.9 shows the predicted histograms superimposed with the data spectrum used. The Monte Carlo sample has a higher b flavour content than the data as a HEPG filter was applied when it was produced. After tagging the sample is a pure b sample which is consistent with what one would expect as the jet has been tagged using both a lepton and secondary vertex tag.

\begin{tabular}{|c|c|c|c|c|c|c|}
\hline & \multirow{2}{*}{$N^{j e t}$} & \multirow{2}{*}{$N^{\text {tag }}$} & \multicolumn{2}{|c|}{$F_{b}^{\text {jet }}$} & \multicolumn{2}{c|}{$F_{b}^{\text {tag }}$} \\
& & & Value & $\chi^{2} /$ d.o.f & Value & $\chi^{2} /$ d.o.f \\
\hline Monte Carlo & 6376 & 1629 & $0.91 \pm 0.03$ & 0.09 & $0.97 \pm 0.03$ & 1.28 \\
\hline Data & 13250 & 2055 & $0.73 \pm 0.03$ & 2.41 & $1.00 \pm 0.02$ & 0.80 \\
\hline \hline
\end{tabular}

Table 5.1: Summary of the results of the template fits. 


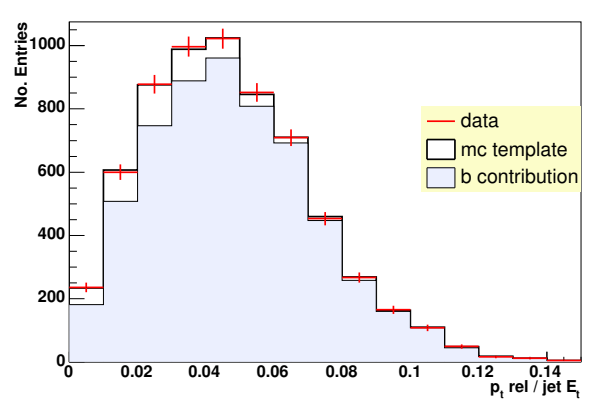

(a) Monte Carlo $p_{t}^{\text {rel }}$ spectrum

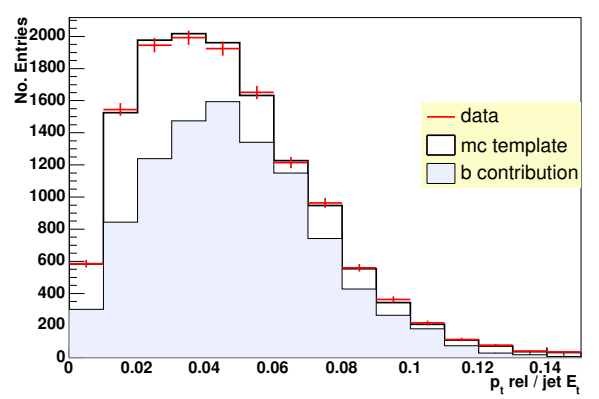

(c) Data $p_{t}^{\text {rel }}$ spectrum

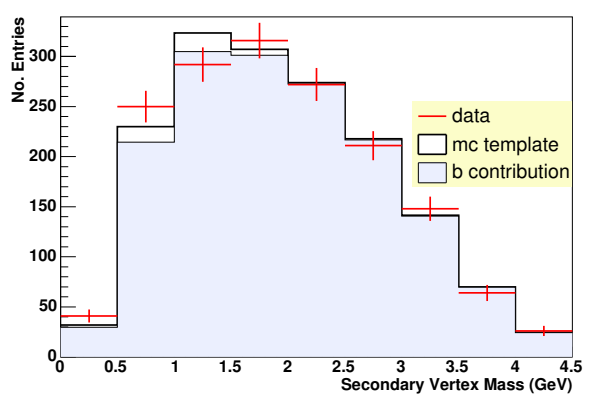

(b) Monte Carlo Mass spectrum

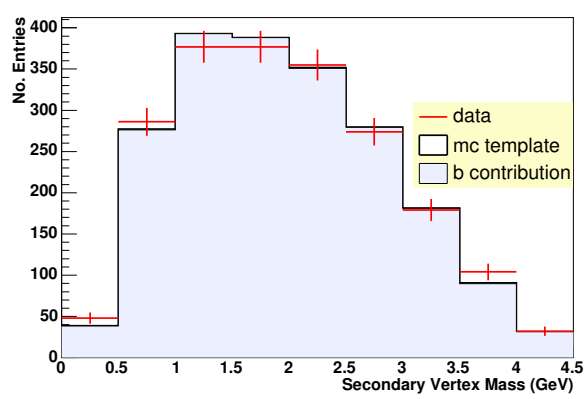

(d) Data Mass spectrum

Figure 5.9: Plots showing predicted histograms from TFractionFitter and estimated b contributions overlaid onto the spectrum used for data and Monte Carlo samples. The $p_{t}^{\text {rel }}$ spectrum is used for $F_{b}^{\text {jet }}$. The secondary vertex mass of the tagged electron jets is used for $F_{b}^{\text {tag }}$. 
For Monte Carlo the tagging efficiency is also calculated using the Monte Carlo truth (HEPG) information. B hadrons are associated to electron jets by requiring the the $\Delta R$ separation between the two is less than 0.7 . The ratio of SECVTX tagged b electron jets to the total number of b electron jets is defined as the efficiency to tag a $b$ jet.

For Monte Carlo the tagging efficiency is measured to be $0.272 \pm 0.013$ (stat) and is in agreement with the value calculated using HEPG information of $0.279 \pm 0.006$. This gives confidence that the method works and there is no inherent bias. In data the tagging efficiency is found to be $0.211 \pm 0.011$ (stat). $\epsilon_{b}$ is plotted as a function of jet $E_{t}, \Delta \phi$, dijet mass and jet $\eta$ in figure 5.10 . It shows the tagging efficiency is only dependent on jet $E_{t}$ in data. Other than the $\eta$ dependence, both Monte Carlo and data show the same trend, with Monte Carlo consistently higher than data. The dependence on jet $E_{t}$ is fitted using equation 5.4 where $P_{1}, P_{2}$ and $P_{3}$ are free parameters.

$$
\epsilon_{b}=\frac{P_{1}}{\left(1+e^{-P_{2} *\left(E_{t}-P_{3}\right)}\right)}
$$

The values of the fit parameters $P_{1}, P_{2}$ and $P_{3}$ are $0.26 \pm 0.04,0.20 \pm 0.10$ $\mathrm{GeV}^{-1}$ and $15.3 \pm 2.0 \mathrm{GeV}$ for data. The values for the fit to Monte Carlo are $0.34 \pm 0.09,0.12 \pm 0.14 \mathrm{GeV}^{-1}$ and $10.1 \pm 6.0 \mathrm{GeV}$, respectively. Equation 5.4 is used to estimate the tagging efficiency for $\mathrm{b}$ jets in the cross section calculation.

The plots in figure 5.11 show both Pythia and Herwig samples exhibit the same $\eta$ dependence. Figure 5.12 shows the $\eta$ dependence for a sample with recently improved detector simulation which provides a better representation of the data. The efficiency measured in data is used for the cross section measurement, therefore the poor simulation of the eta dependence does not 


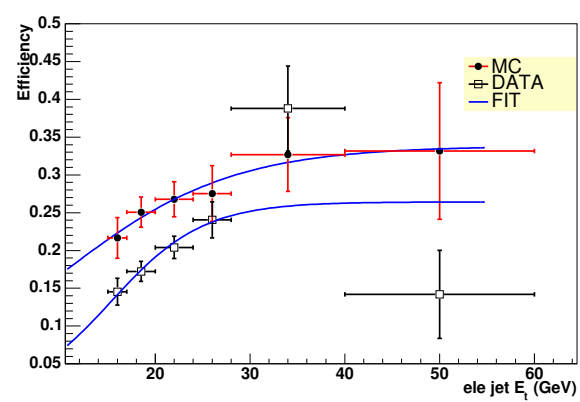

(a) Jet $E_{t}$

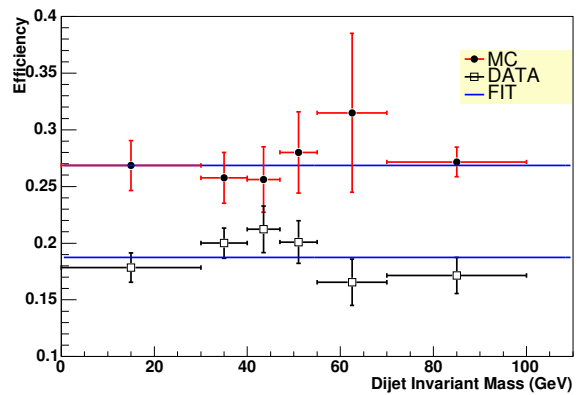

(c) Dijet Mass

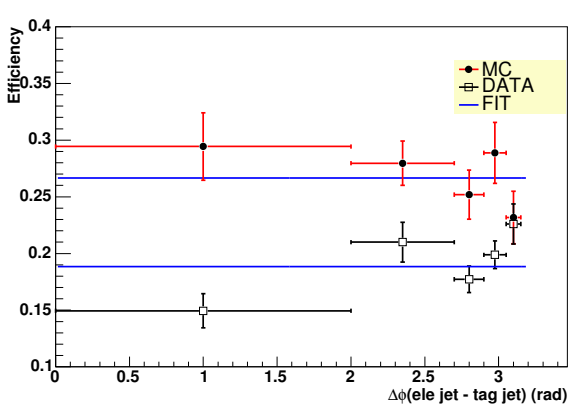

(b) $\Delta \phi$

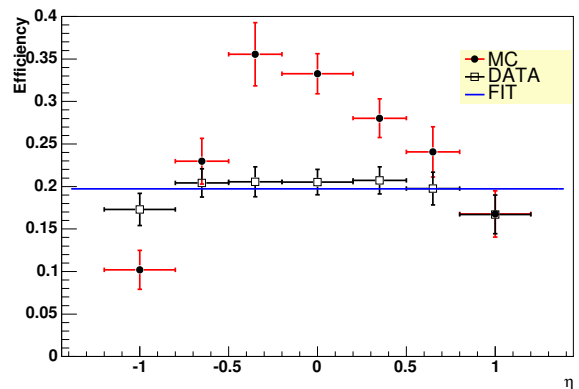

(d) Jet $\eta$

Figure 5.10: $\epsilon_{b}$ as a function of a) Jet Et, b) $\Delta \phi$, c) dijet mass and d)jet $\eta$. For the $\eta$ plots only the data are fitted. 
affect the measurement.

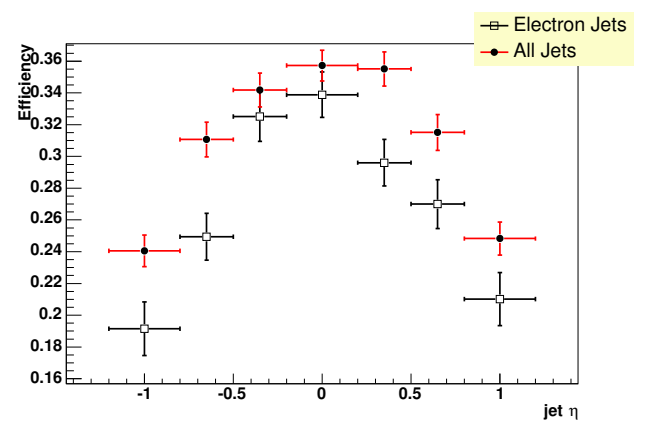

(a) Herwig

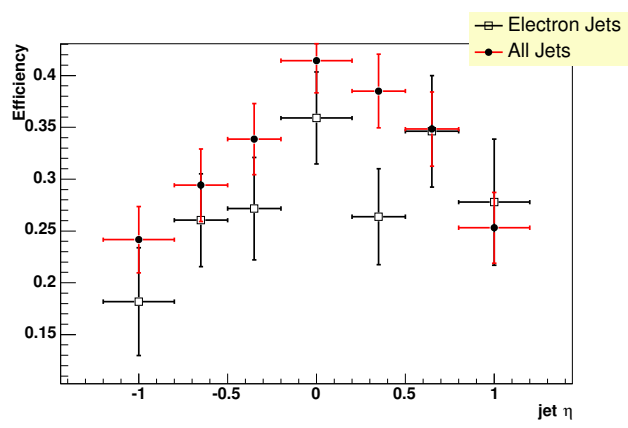

(b) Pythia

Figure 5.11: True $\epsilon_{b}$, found using HEPG information, as a function of $\eta$ for a) Herwig and b) Pythia Monte Carlo samples.

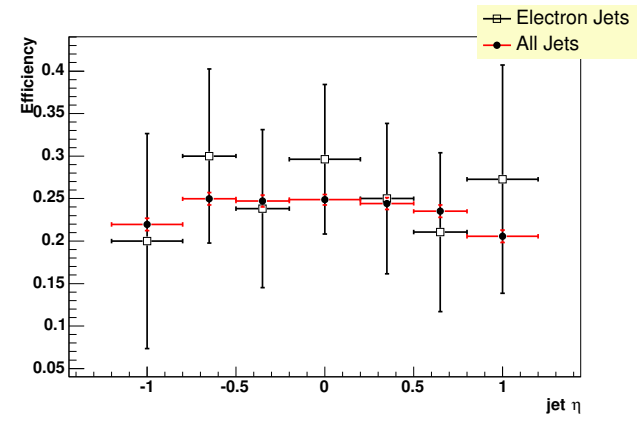

Figure 5.12: True $\epsilon_{b}$, found using HEPG information, as a function of $\eta$ for a Pythia Monte Carlo sample with improved detector simulation. 


\subsubsection{Differences Between Filtered and Unfiltered Data Samples}

The tagging efficiency is measured for semi-leptonic b decays. The plots in figures 5.11 and 5.13 , show the tagging efficiency for all decays is higher than that for semi-leptonic decays. This is also seen in figure 5.14, where the efficiency for the filtered and unfiltered Monte Carlo samples is compared. The filtered samples have a HEPG level requirement applied to them during generation, described in section 5.4.1, the unfiltered samples have no requirements. When a HEPG electron requirement is placed on the unfiltered sample the resultant efficiencies agree with the filtered samples. The same effect is seen with Pythia and Herwig.

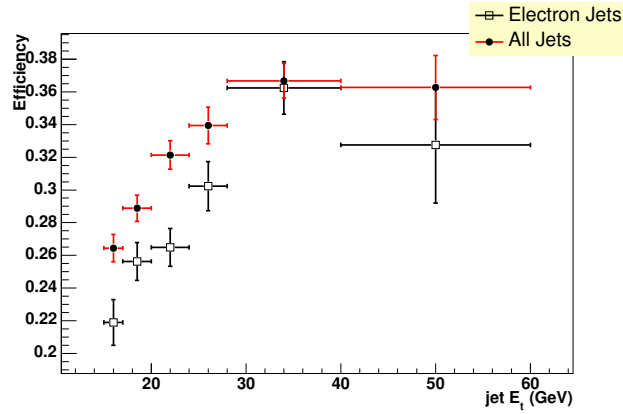

(a) Herwig

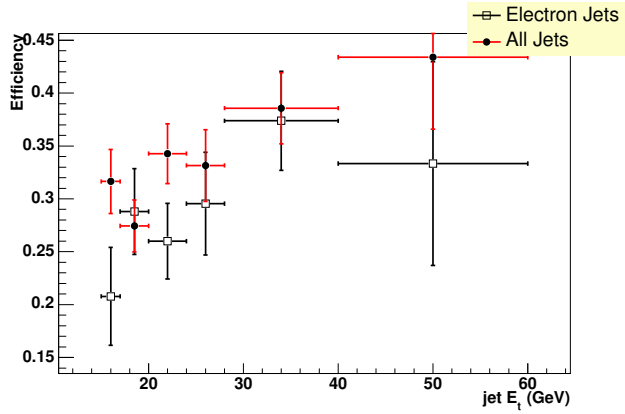

(b) Pythia

Figure 5.13: True $\epsilon_{b}$, found using HEPG information, as a function of jet $E_{t}$ for a) Herwig and b) Pythia Monte Carlo samples.

Figure 5.15 shows the distribution for the number of tracks above $1 \mathrm{GeV}$ within $\Delta R<0.7$ of a $\mathrm{b}$ jet. It indicates that on average there are fewer tracks in electron jets. Figure 5.16 shows that this results in fewer good 


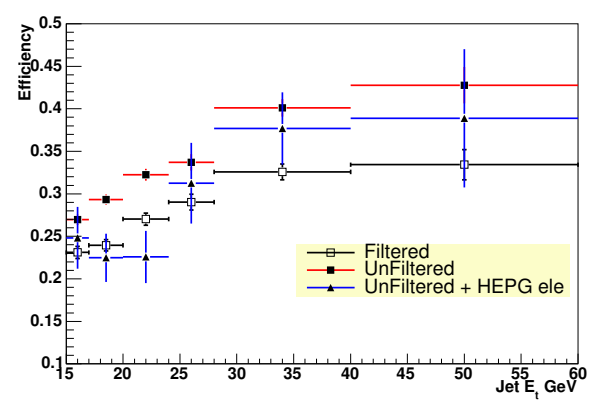

(a) Herwig

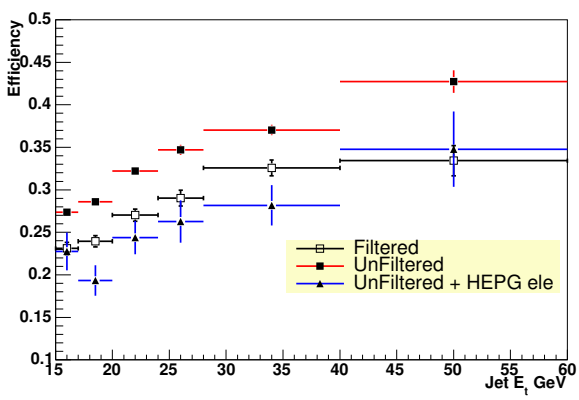

(b) Pythia

Figure 5.14: True $\epsilon_{b}$, found using HEPG information, as a function of jet $E_{t}$ for HEPG filtered and unfiltered samples.

tracks associated with $\mathrm{b}$ jets. A tag requires at least two good tracks, hence the efficiency is lower if there are fewer good tracks associated with the jets.

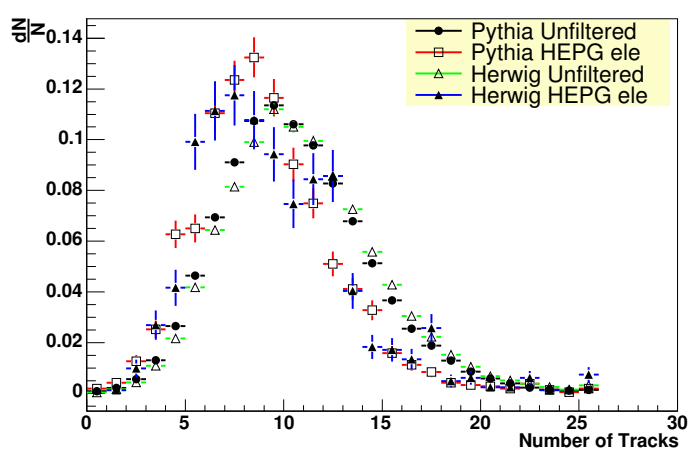

Figure 5.15: Number of tracks with $p_{t}>1 \mathrm{GeV}$ within a $b$ jet for unfiltered and HEPG electron filtered events, all histograms are normalised to unit area.

Electron jets in the electron triggered data sample are compared to electron jets in the filtered Monte Carlo samples and jets in a jet triggered sample. 


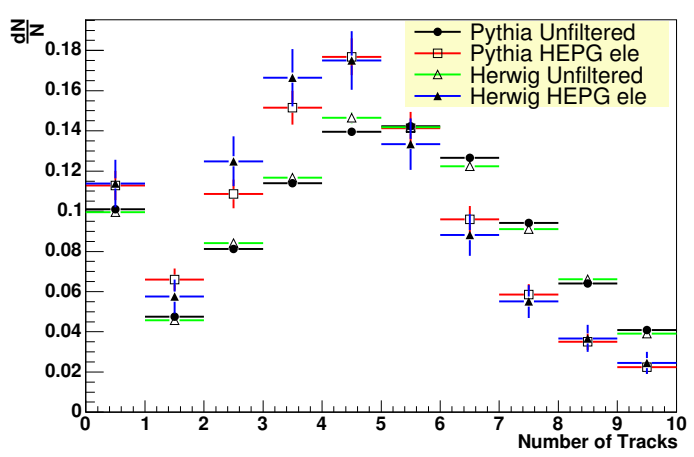

Figure 5.16: Number of good tracks within a b jet for unfiltered and HEPG electron filtered events, all histograms are normalised to unit area.

The number of tracks within a cone of 0.4 of a jet is shown in figure 5.17 . The number of tracks within a jet is, on average, lower for the electron triggered dataset, which shows better agreement with the filtered Monte Carlo.

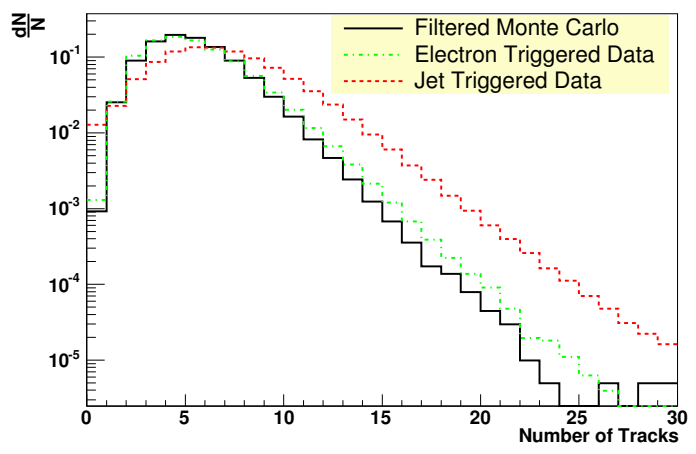

Figure 5.17: Number of tracks with $p_{t}>1 \mathrm{GeV}$ within a jet for HEPG filtered Monte Carlo and electron triggered and jet triggered datasets. Histograms are normalised to unit area.

To account for this effect the ratio of efficiencies between the unfiltered 
and filtered Monte Carlo samples is used as a correction factor, this is shown in figure 5.18, the fit to a constant gives a value of $1.184 \pm 0.017$ for the correction. The correction is applied to the efficiency found in data to account for the fact that the efficiency is found for semi-leptonic b decays, where as the cross section calculation is for jets with all type of b decay.

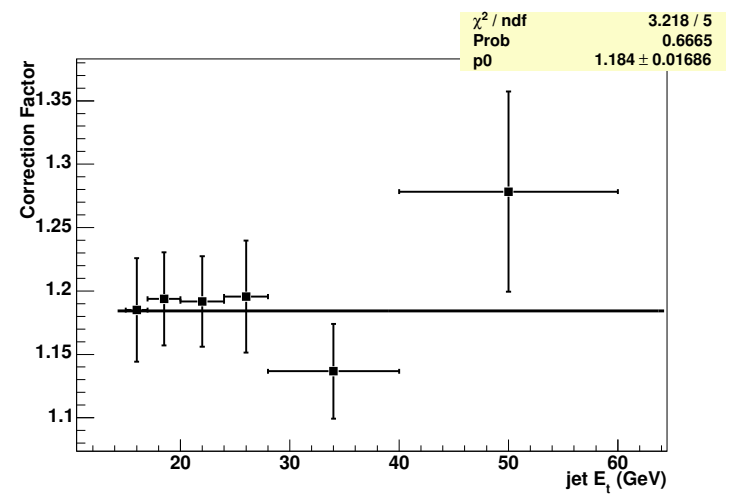

Figure 5.18: Correction factor as a function of jet $E_{t}$ to account for the difference in unfiltered and filtered Monte Carlo samples. The line represents a fit to a constant function, the fit parameters are shown in the top right corner.

\subsubsection{Systematic Uncertainty}

The sources of systematic uncertainty considered are: uncertainties in calculating the purity; the uncertainty due to the correction factor used to account for non semi-leptonic decays; the uncertainty for the fit to the $E_{t}$ dependence.

The templates may bias the purity measurement and are a source of uncertainty. This is quantified using the secondary vertex mass templates of the away jets. The $\mathrm{b}$ fraction before applying SECVTX is found by fitting to 
the secondary vertex mass spectrum of the away jets, the tagging efficiency is calculated to be 0.218 ; the difference between this and the value found using the $p_{t}^{r e l}$ spectra of the electron jet is $3.5 \%$ and is taken as an estimate for the systematic uncertainty.

To account for the uncertainty in the correction factor, half the difference between the value and 1 is taken as the systematic uncertainty, an uncertainty of $7.7 \%$. This is combined in quadrature with the purity systematic to give a total systematic uncertainty of $8.5 \%$.

A systematic uncertainty is also applied for the fit to the efficiency as a function of jet Et. The covariance matrix returned for the fit parameters is used to find the error on the efficiency. This is shown in figure 5.19 and is combined in quadrature with the other systematics.

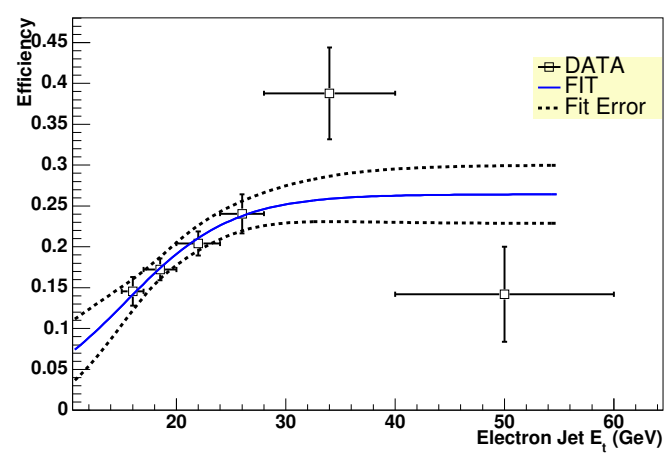

Figure 5.19: SECVTX tagging efficiency in data, together with the fit and corresponding error. 


\subsection{Use in the Cross Section Measurement}

The parameterisation of the efficiency in data as a function of jet $E_{t}$ (equation 5.4. figure 5.19 is used for the cross section measurement. The average $E_{t}$ of jets in the cross section data sample is found and the corresponding efficiency is calculated. This is then multiplied by the correction factor described in section 5.4.3 to account for the difference between tagging efficiency for electron jets and all jets. 


\section{Chapter 6}

\section{Data Samples and Event}

\section{Selection}

This chapter discusses the data samples and event selection used for the cross section calculation. The trigger used and its efficiency are described in section 6.1. The various Monte Carlo samples used for theoretical predictions are discussed in section 6.2. The event selection criteria and related acceptance and purity measurements are explained in sections 6.3, 6.4 and 6.5, respectively.

\subsection{Data Samples}

The data sample consists of events acquired using a $20 \mathrm{GeV}$ jet trigger. At level 1 a single tower with $E_{t}$ greater than $5 \mathrm{GeV}$ is required and is prescaled so that only 1 in 20 such events are triggered ${ }^{1}$. At level 2, candidate events must contain a cluster with $E_{t}$ greater than $15 \mathrm{GeV}$. A prescale is also applied

\footnotetext{
${ }^{1} \mathrm{~A}$ prescale value of 20 .
} 
here - initially a value of 12 , which was later increased to 25. At level 3, a candidate event must contain a jet with $E_{t}$ greater than $20 \mathrm{GeV}$. An effective prescale of 311.61 is calculated for the level 1 and level 2 prescales[52]. Only runs marked as "good" during data taking are used, in addition the silicon detector is required to be operational during the run. The luminosity of the data sample after good run selection is $64.5 \pm 3.9 \mathrm{pb}^{-1}$. The error represents the systematic uncertainty, which is a combination of the uncertainty in the $p \bar{p}$ cross section [53] and the uncertainty in the luminosity monitor [37.

\subsubsection{Trigger Efficiency}

The trigger efficiency calculated for the CDF inclusive jet cross section measurement [52] is used. The efficiency is measured using a dataset obtained with a lower jet $E_{t}$ trigger threshold. For events containing a jet with $E_{t}$ greater than $20 \mathrm{GeV}$, the trigger record stored for each event is used to find whether the event was accepted by the $20 \mathrm{GeV}$ jet trigger. The trigger efficiency is defined as the ratio of events accepted by the $20 \mathrm{GeV}$ jet trigger to the total number of $20 \mathrm{GeV}$ jet events in the sample. The efficiency to trigger on events containing a $20 \mathrm{GeV}$ jet was measured as function of the $E_{t}$ of the jet ${ }^{2}$. Equation 6.1 parametrises the efficiency and is used to fold the trigger efficiency into the acceptance calculation.

$$
\epsilon_{\text {trig }}=\frac{0.9979}{1+e^{-0.247 *\left(E_{t}-18.94\right)}}
$$

\footnotetext{
${ }^{2}$ For the measurement the jet $E_{t}$ was corrected by a factor of 1.042 to account for the Run II-Run I jet energy scale difference.
} 


\subsection{Monte Carlo Samples}

Pythia and Herwig Monte Carlo samples $\mathrm{3}^{3}$ simulating generic $2 \rightarrow 2$ processes are used to find Leading Order predictions for the cross section. The $p t_{\min }$ of the hard scatter is set to $18 \mathrm{GeV}$ and CTEQ5l PDFs are used (see section 2.5.1. To compare to Next to Leading Order a MC@NLO $b \bar{b}$ sample is used. Table 6.1 lists the numbers of events generated. As well as generating events the Monte Carlo generators also calculate the cross section for the processes generated using Monte Carlo integration techniques [9], these are also listed in table 6.1. The ratio of the number of events generated to the cross section for the generated processes is equivalent to the luminosity of the sample and can be used to normalise the various Monte Carlo samples to one another as well as data.

\begin{tabular}{|c|c|c|}
\hline Monte Carlo & Number of Events Generated & Cross Section $(\mu \mathrm{b})$ \\
\hline Pythia & 4977540 & 49.13 \\
\hline Herwig & 1990000 & 39.75 \\
\hline MC@NLO & 7154050 & 48.23 \\
\hline \hline
\end{tabular}

Table 6.1: Number of events generated and the cross section of the generated process for each of the Monte Carlo samples.

To increase the statistical accuracy of predictions and prevent any bias from the analysis procedure, reconstructed hadron jets are used to calculate the Monte Carlo cross sections. The number of events with at least two b jets within an $|\eta|<1.2$, where one of the jets has $E_{t}>30 \mathrm{GeV}$ and the

\footnotetext{
${ }^{3}$ The underlying event in Pythia is tuned using CDF minimum bias events [54]. There is no such tuning for Herwig.
} 
other jet has $E_{t}>20 \mathrm{GeV}$, are counted $\left(N_{b \bar{b}}\right)$. The cross section prediction is found using equation 6.2 where $N_{\text {tot }}$ is the number of events generated and $\sigma_{g e n}$ is the cross section of the generated process.

$$
\sigma_{b \bar{b}}^{\text {pred }}=\frac{N_{b \bar{b}}}{N_{t o t}} \sigma_{g e n}
$$

\subsection{Event Selection}

Candidate events are required to have at least two positively tagged SECVTX jets within an $|\eta|<1.2$. One of the tagged jets is required to have corrected transverse energy $\left(E_{t}^{c o r}\right)$ greater than $30 \mathrm{GeV}$ and the other tagged jet is required to have $E_{t}^{c o r}$ greater than $20 \mathrm{GeV}$. Figure 6.1 shows comparisons between data and Pythia for various kinematic distributions after event selection; for data each event is weighted by the trigger efficiency for the event. There is good agreement between data and Monte Carlo for the $\phi$ and $\eta$ distributions. The agreement for $E_{t}$ is good aside from the first bin, the normalisation includes all bins. In figures 6.2 (a), (b) the normalisation does not include the first bin and illustrates that the first bin is affected by the trigger turn on.

The azimuthal angle, $\Delta \phi$, between the two jets is plotted in figure 6.3 , which shows good agreement between data and Monte Carlo predictions. This demonstrates that the event selection picks out predominantly back-toback jets and so the final event sample will be enriched with Leading Order flavour creation events. 

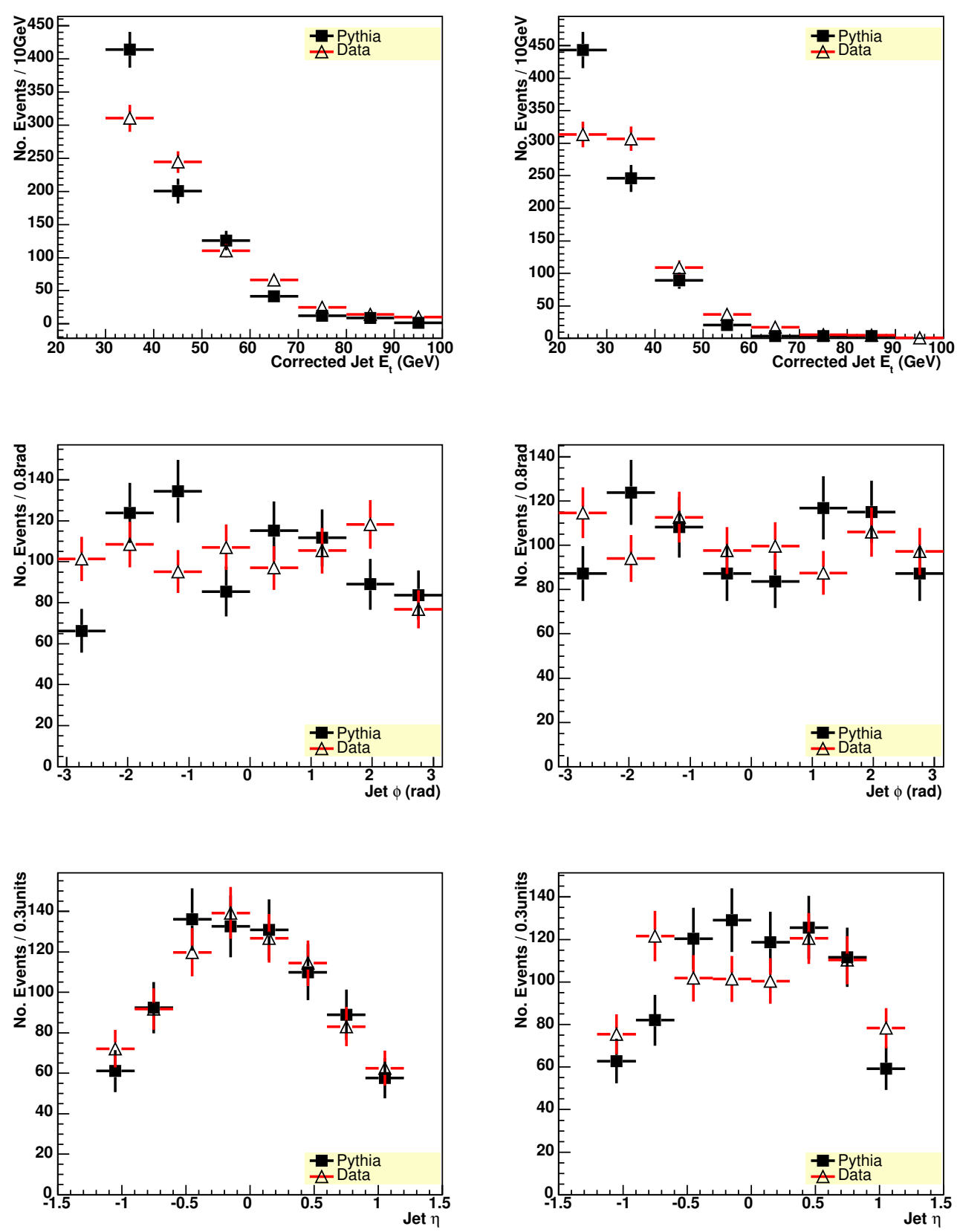

Figure 6.1: Distributions of $E_{t}^{\text {cor }}$ (top), $\phi$ (middle) and $\eta$ (bottom) for the leading tagged jet (left) and second tagged jet (right). All Monte Carlo histograms are normalised to the number of events in data. 


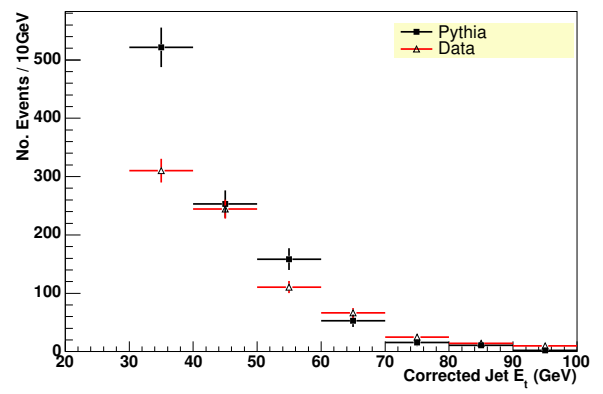

(a) leading jet

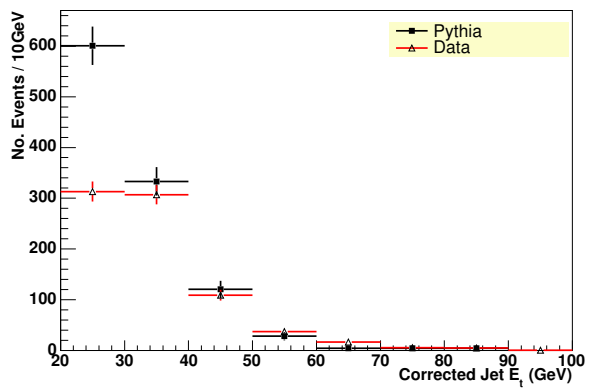

(b) second jet

Figure 6.2: Distributions of $E_{t}^{c o r}$ for a) the leading tagged jet and b) the second tagged jet. In both cases the first bin is excluded from the Monte Carlo normalisation. 


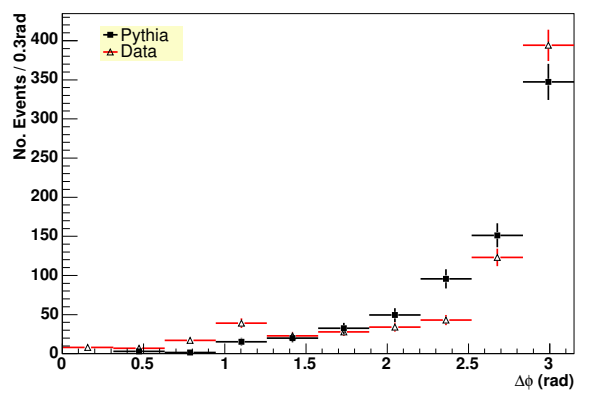

(a) Pythia

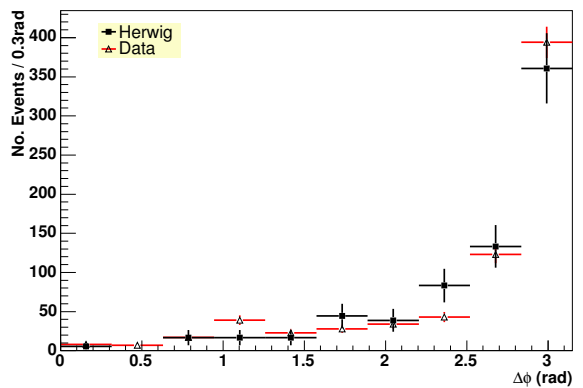

(b) Herwig

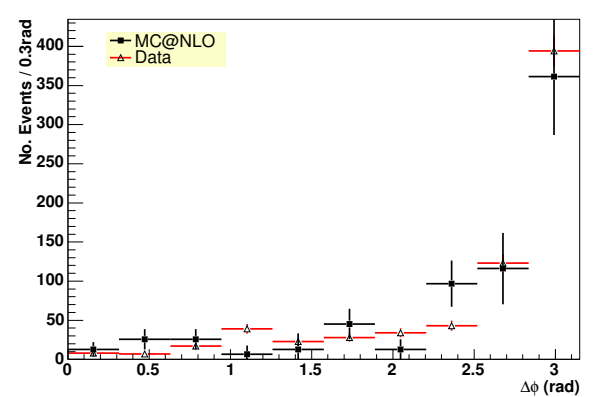

(c) MC@NLO

Figure 6.3: Comparisons between data and a) Pythia, b) Herwig and c) $M C @ N L O$ for the $\Delta \phi$ distribution. Monte Carlo histograms are normalised to the number of events in data. 


\subsection{Acceptance}

The event selection requires two jets, one with $E_{t}^{c o r}$ greater than $30 \mathrm{GeV}\left(E_{t}^{1}\right)$ and the other with $E_{t}^{c o r}$ greater than $20 \mathrm{GeV}\left(E_{t}^{2}\right)$, with both jets lying in the central region. Figure 6.4 shows that the difference between the hadron jet $E_{t}$ and the corrected calorimeter jet $E_{t}$ can be as large as $40 \mathrm{GeV} / 4$. The smearing will result in events being accepted despite true jet energies being less than the required threshold and events being rejected despite the true jet energies being above the thresholds. The calculated acceptance quantifies these effects.

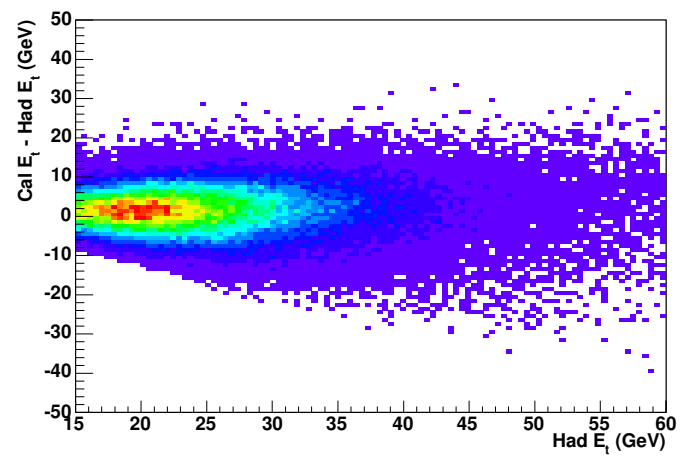

Figure 6.4: The difference in $E_{t}$ between geometrically matched hadron (Had) and corrected calorimeter (Cal) jets, shown as a function of hadron jet $E_{t}$.

After event selection the sample contains three classes of events:

- Events where the calorimeter jet energies are above the cuts and the true jet energies are also above the required cuts $\left(N_{h g}^{c g}\right)$.

- Events where the calorimeter jet energies are above the cuts and the

\footnotetext{
${ }^{4}$ The jets are matched by requiring $\Delta R<0.2$.
} 
true energy of at least one of the jets was below the required cut $\left(N_{h b}^{c g}\right)$.

- Events where the energy of at least one of the calorimeter jets is below the cuts and the true jet energies were above the required cuts $\left(N_{h g}^{c b}\right)$.

The total number of reconstructed events $\left(N^{r e c}\right)$ that pass the cuts is then $N_{h g}^{c g}+N_{h b}^{c g}$ and the total number of events $\left(N^{g e n}\right)$ where the true energies were above the cuts is given by $N_{h g}^{c g}+N_{h g}^{c b}$. The acceptance, A, is defined as:

$$
A=\frac{N^{r e c}}{N^{g e n}}
$$

Using the Pythia sample, the number of events where hadron b jets satisfy the selection criteria, $N^{g e n}$, and the number of events where corrected calorimeter b jets satisfy the selection criteria, $N^{r e c}$ are counted. The trigger efficiency is folded into the calculation by using the $E_{t}$ of the leading jet for each event and equation 6.1 to calculate the trigger efficiency for the event. Each calorimeter event is then weighted by the trigger efficiency for the event. The modified acceptance, $A_{\text {trig }}$, is given by equation 6.4 and is calculated to be $1.03 \pm 0.02$. If the trigger efficiency is not included the acceptance is $1.21 \pm 0.02$.

$$
A_{\text {trig }}=\frac{\sum_{N^{r e c}} \epsilon_{\text {trig }}(\text { lead jet })}{N^{g e n}}
$$

A value of $A_{\text {trig }}$ greater than one indicates that more reconstructed events pass the cuts compared to generated events. Figure 6.5 shows the calorimeter jet $E_{t}$ minus the hadron jet $E_{t}$ for matched jets. The mean is always greater than zero, hence it is more likely that hadron jets are reconstructed with a slightly higher energy, and so fall into the acceptance. Above $35 \mathrm{GeV}$ the difference rises with $E_{t}$ which translates into a rise in acceptance as $E_{t}$ cuts are increased, as shown in table 6.2 . 


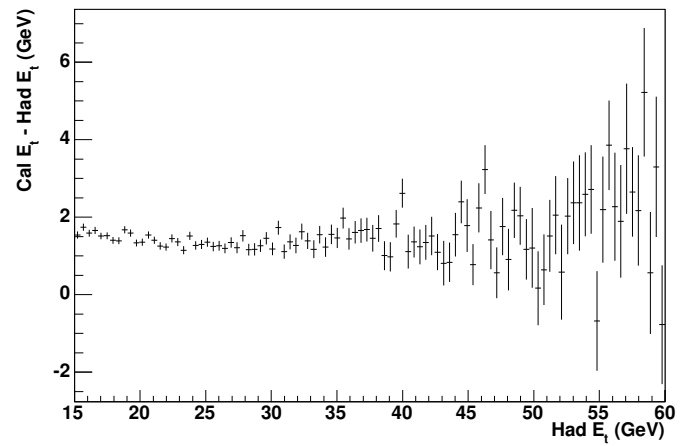

Figure 6.5: Profile plot showing the difference in $E_{t}$ between geometrically matched hadron (Had) and calorimeter (cal) jets, shown as a function of hadron jet $E_{t}$.

\begin{tabular}{|c|c|}
\hline Cuts Used & Acceptance \\
\hline$E_{t}^{1}>30 \mathrm{GeV}, E_{t}^{2}>25 \mathrm{GeV}$ & $1.20 \pm 0.02$ \\
\hline$E_{t}^{1}>35 \mathrm{GeV}, E_{t}^{2}>20 \mathrm{GeV}$ & $1.28 \pm 0.02$ \\
\hline$E_{t}^{1}>35 \mathrm{GeV}, E_{t}^{2}>25 \mathrm{GeV}$ & $1.28 \pm 0.03$ \\
\hline$E_{t}^{1}>40 \mathrm{GeV}, E_{t}^{2}>25 \mathrm{GeV}$ & $1.37 \pm 0.04$ \\
\hline$E_{t}^{1}>40 \mathrm{GeV}, E_{t}^{2}>30 \mathrm{GeV}$ & $1.31 \pm 0.04$ \\
\hline$E_{t}^{1}>50 \mathrm{GeV}, E_{t}^{2}>35 \mathrm{GeV}$ & $1.36 \pm 0.07$ \\
\hline \hline
\end{tabular}

Table 6.2: Change in acceptance as the jet $E_{t}$ selection cuts are increased. 
The acceptance is calculated as a function of the leading jet $E_{t} ; \Delta \phi$ between the leading jet and second jet; and the dijet invariant mass, respectively. $N^{r e c}\left(N^{g e n}\right)$ is binned in terms of the measured calorimeter (hadron) jet values and equation 6.4 used to calculate the acceptance for each bin. Figure 6.6 shows the acceptance as a function of the leading jet $E_{t}$, the dijet invariant mass and $\Delta \phi$. The dependence on $E_{t}$ and dijet mass is fitted using equation 5.4 a first order polynomial fit is used to describe the dependence on $\Delta \phi$. The values for the free parameters are listed in table 6.3.

\begin{tabular}{|c|c|c|c|}
\hline Parameter & $E_{t}$ & dijet mass & $\Delta \phi$ \\
\hline P1 & $1.54 \pm 0.20$ & $1.67 \pm 0.17$ & $1.43 \pm 0.13$ \\
\hline P2 & $0.118 \pm 0.072 \mathrm{GeV}^{-1}$ & $0.059 \pm 0.025 \mathrm{GeV}^{-1}$ & $-0.158 \pm 0.048 \mathrm{rad}^{-1}$ \\
\hline $\mathrm{P} 3$ & $33.4 \pm 2.4 \mathrm{GeV}$ & $61.5 \pm 5.3 \mathrm{GeV}$ & - \\
\hline \hline
\end{tabular}

Table 6.3: Parameters for fits to the acceptance versus $E_{t}$ and dijet mass.

\subsubsection{Systematic Uncertainty}

Source of systematic uncertainty for the acceptance calculation include the procedure used, the jet energy corrections applied and the PDFs used in the generation of the sample.

To cross check the procedure, $A_{\text {trig }}$ is calculated using the Herwig sample and is found to be $0.98 \pm 0.04$. This is in good agreement with Pythia and so a systematic uncertainty is not applied for the procedure used.

\footnotetext{
${ }^{5}$ The same expression was also used to fit the $E_{t}$ dependence of the tagging efficiency in section 5.4 .2
} 


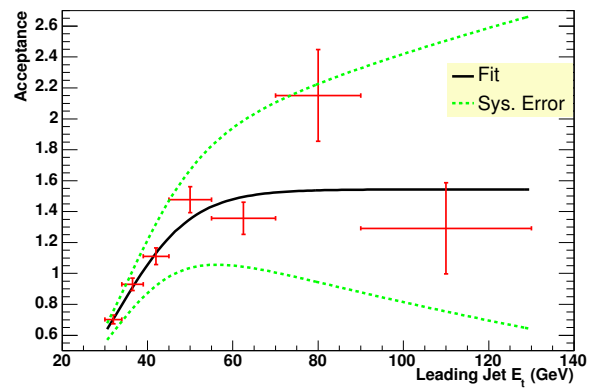

(a) Acceptance v. Leading Jet $E_{t}$

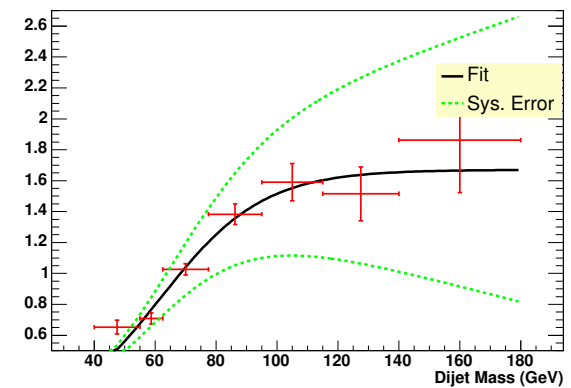

(b) Acceptance v. Dijet Mass

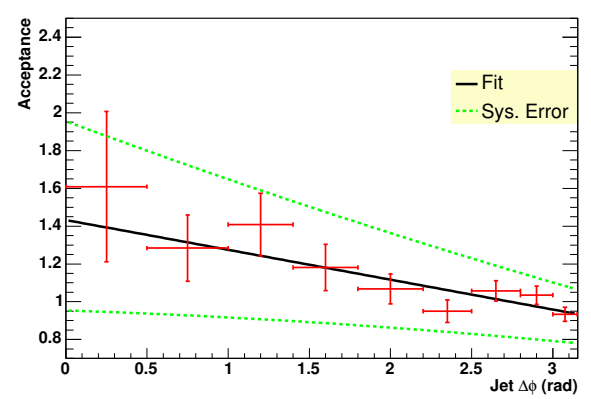

(c) Acceptance v. $\Delta \phi$

Figure 6.6: Acceptance as a function of: a) the leading jet $E_{t}$; b) the dijet invariant mass of the leading jet and second jet; c) $\Delta \phi$ between the leading jet and second jet. The systematic uncertainties are discussed in section 6.4.1. 
Changes to the jet energy corrections will change $N^{r e c}$ and are a source of systematic uncertainty. Each correction is modified by $\pm 1 \sigma$ and the percentage change in $N^{r e c}$ is taken as the systematic uncertainty. The values are shown in table 6.4. When summed in quadrature this results in a positive systematic error of $18.2 \%$ and a negative systematic error of $19.8 \%$ on the acceptance.

\begin{tabular}{|c|c|c|}
\hline Correction & + systematic & - systematic \\
\hline Relative & $9.3 \%$ & $10.8 \%$ \\
\hline Energy Scale & $12.8 \%$ & $13.7 \%$ \\
\hline Multiple Interaction & 0 & 0 \\
\hline Absolute & $8.1 \%$ & $8.5 \%$ \\
\hline b Jet & $3.9 \%$ & $4.0 \%$ \\
\hline Total & $18.2 \%$ & $19.8 \%$ \\
\hline \hline
\end{tabular}

Table 6.4: Systematic uncertainty on acceptance due to each of the jet energy corrections.

Changes in PDFs will affect the jet energy distributions. The Pythia sample used to calculate the acceptance uses CTEQ 5l PDFs. The acceptance is compared to that obtained using CTEQ 6M, MRST 72, 73, 74, 75 and 76 PDFs [55], shown in table 6.5. Taking into account the statistical errors the MRST 74 sample has the largest deviation from the CTEQ 51 value $(1.15 \sigma)$. The difference between the MRST 74 sample and the CTEQ 51 value is assigned as the systematic uncertainty due to PDFs. It is combined in quadrature with the jet corrections systematic to give a total systematic uncertainty of $+18.7 \%-20.3 \%$ on the acceptance. 


\begin{tabular}{|c|c|}
\hline PDF Set Used & Acceptance \\
\hline CTEQ 6M & $1.263 \pm 0.060$ \\
\hline MRST 72 & $1.242 \pm 0.053$ \\
\hline MRST 73 & $1.265 \pm 0.058$ \\
\hline MRST 74 & $1.152 \pm 0.052$ \\
\hline MRST 75 & $1.178 \pm 0.051$ \\
\hline MRST 76 & $1.188 \pm 0.055$ \\
\hline \hline
\end{tabular}

Table 6.5: Calculated acceptance for various PDF sets.

For acceptance as a function of jet $E_{t}$, dijet mass and $\Delta \phi$ the systematic uncertainty is calculated for each bin separately, since the jet correction systematic uncertainties are $E_{t}$ dependent.

\subsection{Purity of Sample}

The technique described in section 5.3 .3 is used to find the b flavour content of the sample, using the secondary vertex mass to discriminate between $\mathrm{b}$ and c, uds production. Templates are made using the Pythia and Herwig $2 \rightarrow 2$ process samples described in section 6.1 and are shown in figure 6.7.

The $\mathrm{b}$ fraction in data is extracted by fitting the templates to the secondary vertex mass spectrum of the leading and second tagged jets. Table 6.6 shows the results of the fitting for the leading and second jets separately. Figure 6.8 shows the secondary vertex mass spectrum in data with the predicted histogram from the fitter and estimated $\mathrm{b}$ contribution. There is good agreement between data and the predicted Monte Carlo spectrum. The er- 


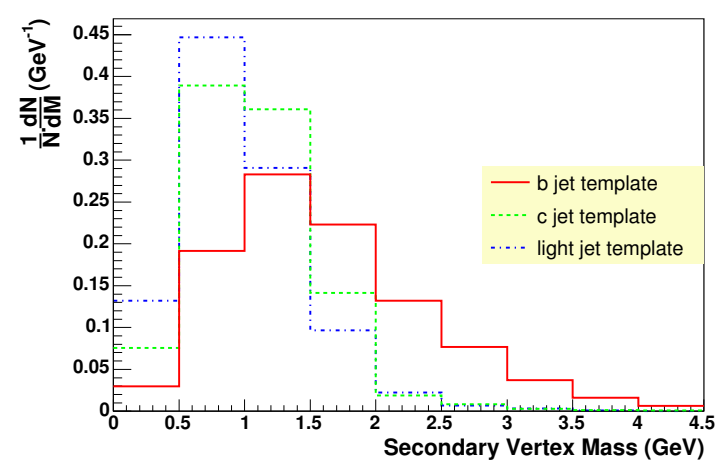

Figure 6.7: Secondary vertex mass spectra used for fits to find purity of the data sample.

ror on the fractions takes into account the statistical uncertainties in both the data and Monte Carlo spectra. There is good agreement between the $\mathrm{b}$ fractions found using the leading and second tagged jet spectra, therefore a combination of the two spectra is used for the final $\mathrm{b}$ fraction. This reduces the statistical uncertainty in data.

\begin{tabular}{|c|c|c|}
\hline Spectrum Used & $\mathrm{b}$ fraction & $\chi^{2} /$ d.o.f \\
\hline Leading tagged jets & $0.86 \pm 0.06$ & 0.20 \\
\hline Second tagged jets & $0.81 \pm 0.06$ & 1.03 \\
\hline \hline
\end{tabular}

Table 6.6: Results of fraction fitting to the secondary vertex mass spectrum of leading and second tagged jets in data.

Figure 6.9 shows the combined mass spectrum in data with the predicted Monte Carlo histogram and estimated b contribution superimposed. The fit returns a b fraction of $0.832 \pm 0.043$ with a $\chi^{2} /$ d.o.f of 0.76 for the fit, the error is used as the statistical error for the cross section. There is good 


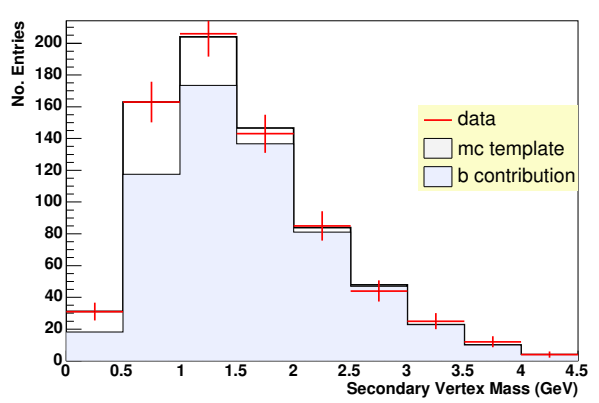

(a) Leading tagged jets

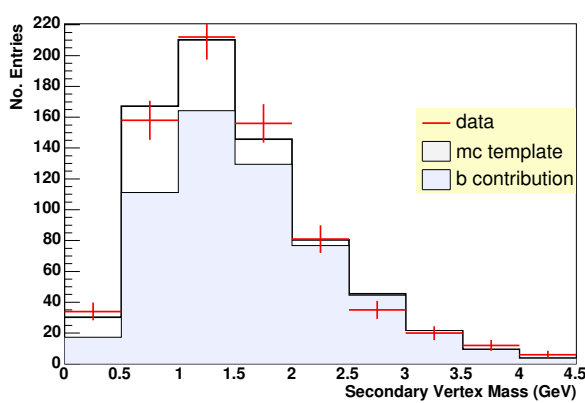

(b) Second tagged jets

Figure 6.8: The secondary vertex mass spectrum in data with the predicted Monte Carlo histogram and estimated $b$ contribution overlaid for the a) Leading tagged jets and b) Second tagged jets.

agreement with the fits to the individual cross sections.

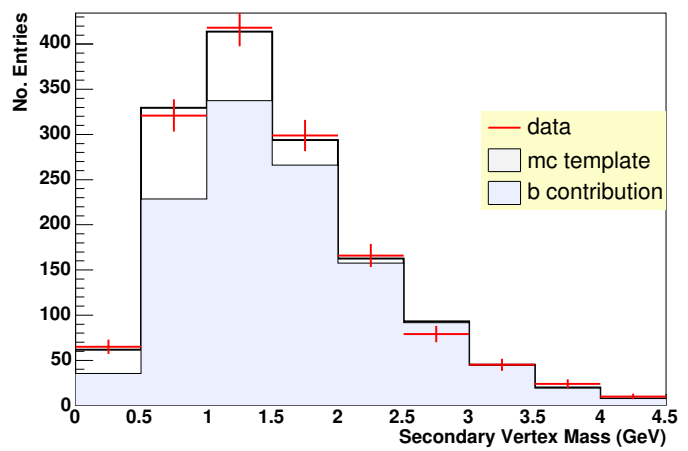

Figure 6.9: The secondary vertex mass spectrum in data with the predicted Monte Carlo histogram and estimated b contribution. 


\subsubsection{Systematic Uncertainties}

The templates used and any dependence of the purity on jet $E_{t}$ contribute to the systematic uncertainty. To find the dependence on the templates the fit to the combined spectrum is performed using the templates derived from Pythia and and Herwig separately. The b fractions in data are $0.830 \pm 0.044$ and $0.839 \pm 0.046$ for Pythia templates and Herwig templates, respectively. The effect of changing templates is negligible and a systematic uncertainty is not applied. For the $E_{t}$ dependence the difference between the combined fit and the separate fits to the leading and second tagged jet spectra is assigned as a systematic uncertainty. 


\section{Chapter 7}

\section{Cross Section Measurement}

This chapter details the measurement of the inclusive $b \bar{b}$ jet production cross section. The measurement is carried out for both $\mathrm{b}$ jets within $|\eta|<1.2$ where one of the jets has an $E_{t}>30 \mathrm{GeV}$ and the other has an $E_{t}>20 \mathrm{GeV}$. Measurements of the differential cross section with respect to: the leading jet $E_{t}$; dijet invariant mass of the two tagged jets, $M_{\bar{b} \bar{b}}$; and the azimuthal angle, $\Delta \phi$, between the two jets are also described. The results are compared to the various Monte Carlo predictions, and the differences are discussed.

\subsection{Inclusive Cross Section}

The formula used to calculate the inclusive cross section is:

$$
\sigma_{b \bar{b}}(|\eta|<1.2)=\frac{N^{e v} F_{b}^{e v}}{\epsilon_{b}^{\text {lead }} \epsilon_{b}^{\text {other }} A_{\text {trig }} \int L}
$$

$N^{e v}$ is the number of events in the sample after event selection and $F_{b}^{e v}$ is the fraction of these events that contain $b$ jets. The efficiency for tagging the leading (second) jet is denoted by $\epsilon_{b}^{\text {lead }}\left(\epsilon_{b}^{\text {other }}\right)$. A $A_{\text {trig }}$ is the event acceptance 
which includes trigger efficiency, calculated using equation 6.4. $\int L$ is the integrated luminosity, which is divided by the effective prescale for the jet trigger.

The tagging efficiency is parametrised as a function of raw jet $E_{t}$. The mean raw $E_{t}$ of all tagged leading jets is found and the efficiency corresponding to this value is calculated using equation 5.3 . The efficiency for the second tagged jet in the events is found in a similar manner.

\subsubsection{Results}

The results for data, the Leading Order Pythia and Herwig predictions and the Next to Leading Order MC@NLO prediction are shown in table 7.1. Data agree with the Pythia prediction, however, the predicted cross section from both Herwig and MC@NLO are lower than Pythia. The discrepancy is discussed in section 7.3 .

\subsection{Differential Cross Section}

The differential cross section is measured as a function of leading jet $E_{t}, M_{b \bar{b}}$ and $\Delta \phi$. For each bin in $E_{t}, M_{b \bar{b}}$ and $\Delta \phi$ the differential cross section is found using equation 7.2 , where $\mathrm{X}$ is the variable under consideration and $\Delta X$ is the bin width.

$$
\frac{d \sigma_{b \bar{b}}}{d X}(|\eta|<1.2)=\frac{N^{e v} F_{b}^{e v}}{\epsilon_{b}^{\text {lead }} \epsilon_{b}^{\text {other }} A_{\text {trig }} \Delta X \int L}
$$

The average raw $E_{t}$ of the leading tagged jets and second tag jets in the bin is used to find $\epsilon_{b}^{\text {lead }}$ and $\epsilon_{b}^{\text {other }}$, respectively. The central value for each 


\begin{tabular}{|c|c|}
\hline Variable & Value \\
\hline$N^{e v}$ & 716 \\
\hline$F_{b}^{e v}$ & 0.83 \\
\hline$\epsilon_{b}^{\text {lead }}$ & 0.31 \\
\hline$\epsilon_{b}^{\text {other }}$ & 0.26 \\
\hline$A_{\text {trig }}$ & 1.03 \\
\hline $\int L$ & $64.5 \mathrm{pb}^{-1}$ \\
\hline \hline$\sigma_{b \bar{b}}(|\eta|<1.2)$ & $34.5 \pm 1.8 \mathrm{nb}$ \\
\hline Pythia $(\mathrm{CTEQ} 5 \mathrm{l}) \sigma$ & $38.7 \pm 0.6 \mathrm{nb}$ \\
\hline Herwig $(\mathrm{CTEQ} 5 \mathrm{l}) \sigma$ & $21.5 \pm 0.7 \mathrm{nb}$ \\
\hline $\mathrm{MC} @ \mathrm{NLO} \sigma$ & $28.5 \pm 0.6 \mathrm{nb}$ \\
\hline \hline
\end{tabular}

Table 7.1: Inputs and results for the cross section calculation in data. Cross section predictions for Pythia, Herwig and MC@NLO are also given. All errors are statistical.

bin is used to find $A_{\text {trig }}$ from the corresponding fit.

\subsubsection{Results}

Figures 7.1 and 7.2 show the differential cross section as a function of $E_{t}$ and $M_{b \bar{b}}$, respectively. There is good agreement between Pythia and data. The agreement between data and MC@NLO improves at higher $E_{t}$ whereas Herwig predictions are all too low.

Figure 7.3 shows the differential cross section as a function of $\Delta \phi$. Data agree with Pythia predictions, although there is some indication of deviation at smaller opening angles. The difference between Leading Order and Next 

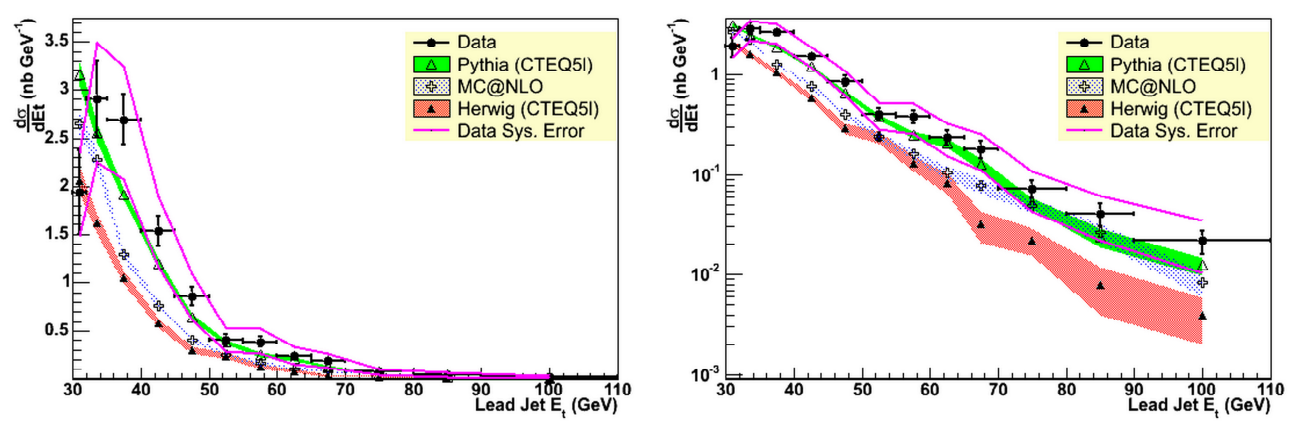

Figure 7.1: The differential cross section as a function of leading jet $E_{t}$, shown on both linear(left) and log(right) scales. The line represents the systematic uncertainty which is described in section 7.4 .
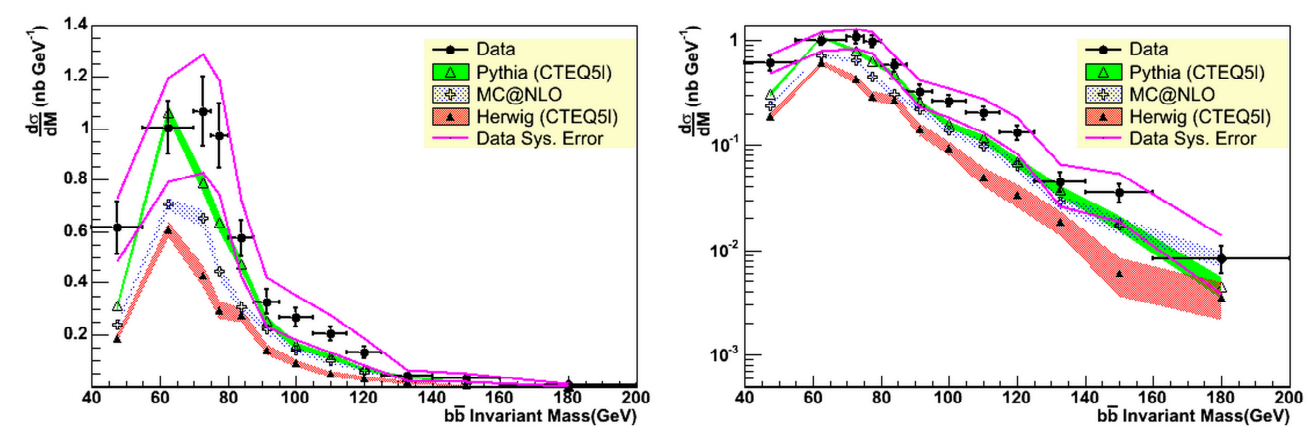

Figure 7.2: The differential cross section as a function of the invariant mass of the two tagged jets, shown on both linear(left) and log(right) scales. The line represents the systematic uncertainty which is described in section 7.4 . 
to Leading Order predictions is highlighted here - for both of the Leading Order Monte Carlos the differential cross section continues to fall as $\Delta \phi$ gets smaller, as the Leading Order process produces back-to-back b jets. However for MC@NLO the differential cross section receives extra contributions at small $\Delta \phi$ as a result of the Next to Leading Order flavour excitation and gluon splitting processes. This agreement is because of the requirement of two central jets in the event selection which picks out the Leading Order flavour creation events. For flavour creation the two b jets are more likely to be scattered into the central region of the detector where as for flavour excitation one the $b$ jets will often go down the beam pipe as only on of the $b$ quarks is involved in the hard scattering. When gluon splitting occurs both b quarks are more likely to end up in the same jet.
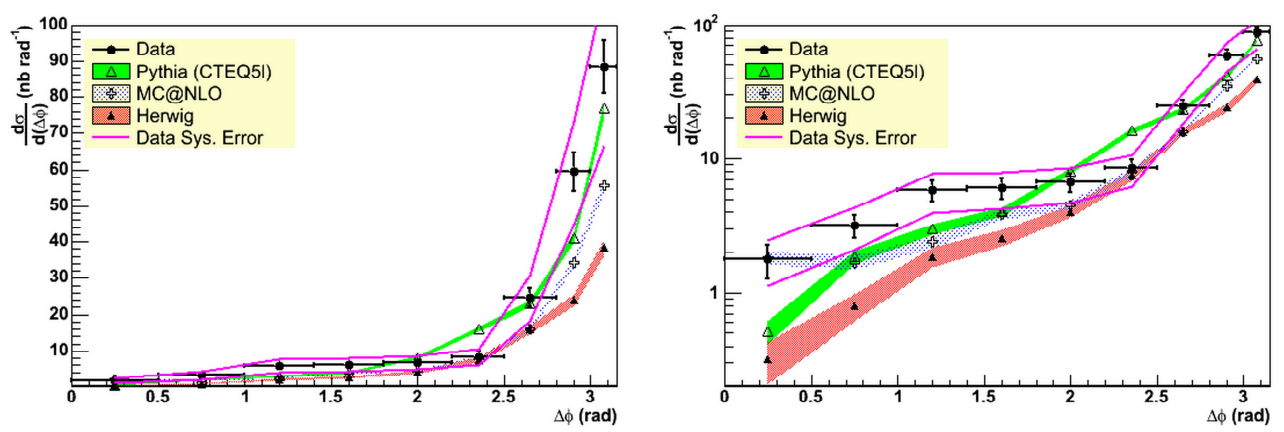

Figure 7.3: The differential cross section as a function of the azimuthal angle $\Delta \phi$ between the tagged jets, shown on both linear(left) and log(right) scales. The line represents the systematic uncertainty which is described in section 7.4. 


\subsection{Differences in Monte Carlo Cross Section Predictions}

Both the MC@NLO and Herwig samples predict a lower cross section than Pythia. Figure 7.4 shows the distribution of leading b jet $E_{t}$ for events with two central b jets, one with $E_{t}$ greater than $30 \mathrm{GeV}$ and the other with $E_{t}$ greater than $20 \mathrm{GeV}$. The distributions are normalised to the luminosity of the Pythia sample and show a clear difference in number of events between Pythia, Herwig and MC@NLO.

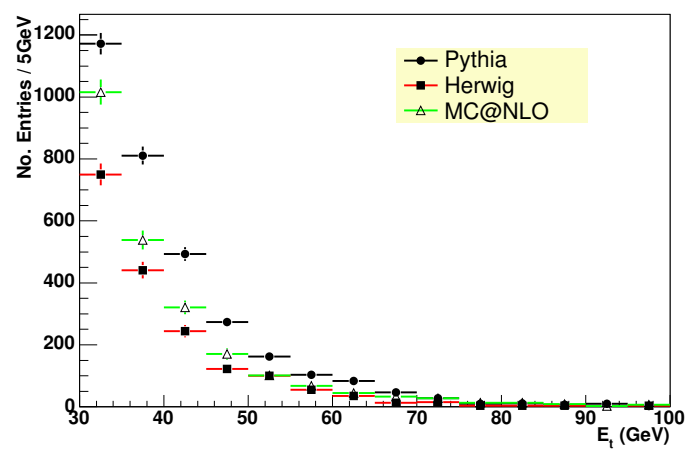

Figure 7.4: The $E_{t}$ of the leading b jet in Pythia, Herwig and MC@NLO data samples. All plots are normalised to the integrated luminosity of the Pythia sample.

Figure $7.5(\mathrm{a})$ shows the leading b hadron $E_{t}$ for events with two central b hadrons, one with $E_{t}$ greater than $30 \mathrm{GeV}$ and the other with $E_{t}$ greater than $20 \mathrm{GeV}$. The distributions are normalised to the luminosity of the Pythia sample. The figure demonstrates that at the b hadron level Pythia and MC@NLO predictions are similar, although Herwig is still lower. 
Since the b hadron $E_{t}$ will be lower than that of a b jet, as a secondary check the leading b hadron $E_{t}$ for events with two b hadrons with $E_{t}>25$ and $E_{t}>15$ is shown in figure $7.5(\mathrm{~b})$, which also shows agreement between Pythia and MC@NLO. The difference in the b jet cross section between Pythia and MC@NLO therefore appears to come from contributions to the jet energy from other processes. One potential cause is contributions from the underlying event. Pythia is tuned to agree with CDF data whereas Herwig and MC@NLO use the default underlying event simulation in Herwig.

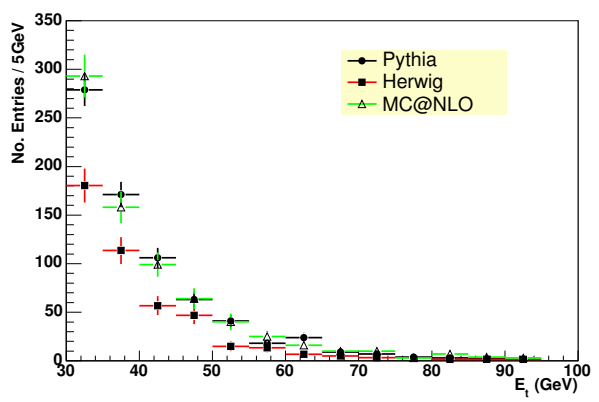

(a)

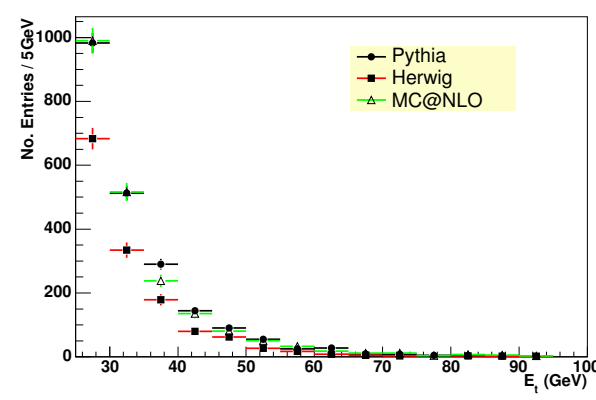

(b)

Figure 7.5: The $E_{t}$ of the leading b hadron in Pythia, Herwig and MC@NLO data samples, all normalised to the integrated luminosity of the Pythia sample. a) For events containing two b hadrons in the central region where one has $E_{t}>30 \mathrm{GeV}$ and the other has $E_{t}>20 \mathrm{GeV}$. b) For events containing two $b$ hadrons in the central region where one has $E_{t}>25 \mathrm{GeV}$ and the other has $E_{t}>15 \mathrm{GeV}$.

The underlying event can be incorporated into Herwig using an external generator such as JIMMY [56]. It generates multi-parton interactions for 
Herwig, and thus provides a simulation of the underlying event 11 Figure 7.6 shows the leading b jet $E_{t}$ spectrum for a MC@NLO sample where JIMMY is used in conjunction with Herwig. The standard energy cuts are used and the MC@NLO+JIMMY sample is normalised to the Pythia luminosity. There is good agreement between the two, indicating that the improved underlying event simulation provided by JIMMY agrees with the tuned Pythia underlying event.

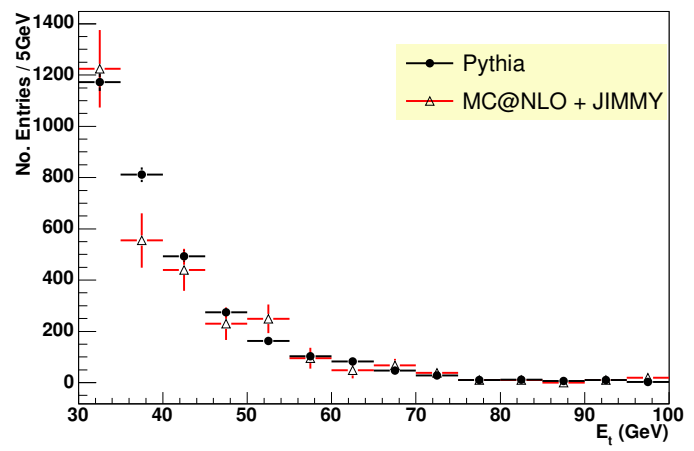

Figure 7.6: The $E_{t}$ of the leading b jet for Pythia and $M C @ N L O+J I M M Y$ normalised to the integrated luminosity of the Pythia sample.

The b jet cross section calculated using the MC@NLO+JIMMY sample is $35.7 \pm 2.0 \mathrm{nb}$ which is in agreement with both data and Pythia. The differential cross sections are shown in figure 7.7. There is good agreement with data in all cases, the differential cross section as a function of $\Delta \phi$ appears to agree better with this sample than with the Leading Order Pythia prediction.

\footnotetext{
${ }^{1}$ The interactions generated are between the soft remnants after the hard scatter. Multiple hard scatters are not generated.
} 


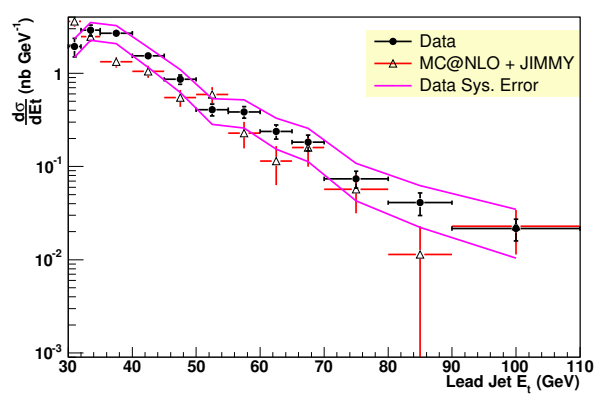

(a) $\frac{d \sigma}{d E_{t}}$

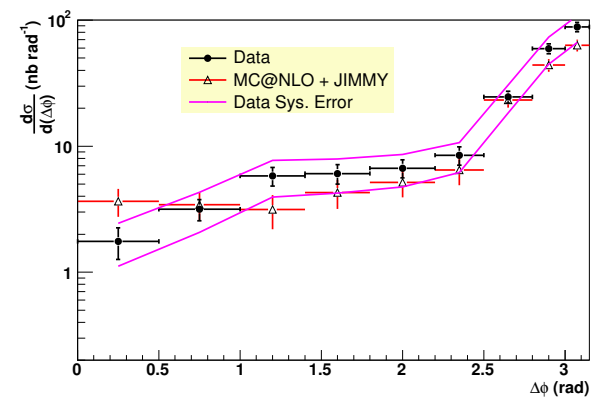

(b) $\frac{d \sigma}{d(\Delta \phi)}$

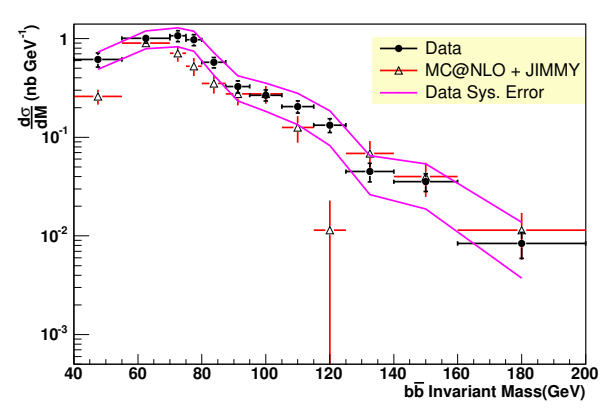

(c) $\frac{d \sigma}{d M_{b \bar{b}}}$

Figure 7.7: The differential cross section as a function of a) leading jet $E_{t}$, b) $\Delta \phi$ and c) $M_{b \bar{b}}$ for data and MC@NLO+JIMMY predictions. 


\subsection{Systematic errors}

Sources of systematic errors on the cross section measurement include: jet corrections, including the $\mathrm{b}$ jet correction; the PDF assumptions in Monte Carlo; acceptance calculation procedure; b tagging efficiency; luminosity; and the $\mathrm{b}$ fraction calculation. These have all been discussed in greater detail earlier and are summarised below.

The errors due to jet corrections and PDFs are incorporated into the acceptance systematic error (section 6.4.1). The uncertainty due to jet corrections are found by finding the change in the number of reconstructed events after adjusting each correction by $\pm 1 \sigma$. All differences are summed in quadrature to give the final systematic due to jet energy corrections. The uncertainty due to PDFs is found by calculating the acceptance using different PDFs and taking the largest difference from the central value. The acceptance calculation is cross checked using a Herwig sample, good agreement is seen and so an uncertainty is not assigned for the procedure used. The total percentage uncertainty on the acceptance is used as the percentage uncertainty on the cross section.

The systematic uncertainty from the b tagging efficiency (section 5.4.4) contains contributions from the $\mathrm{b}$ fraction, the fit used to parametrise the data and the correction factor used to determine the tagging efficiency for all, rather than only semi-leptonic, decays. The uncertainty from each is combined in quadrature and the total percentage uncertainty is used as the percentage uncertainty on the cross section.

The luminosity is assigned the standard CDF value of $\pm 6 \%$, which contains contributions from the uncertainty in the $p \bar{p}$ cross section and the lumni- 
nosity monitor.

The $\mathrm{b}$ fraction is assigned a $\pm 3 \%$ systematic uncertainty (6.5.1), this is the difference between the $\mathrm{b}$ fraction calculated using the combined leading jet and second jet secondary vertex mass spectrum and the fraction calculated using the leading jet and second jet seperately. The contribution to the $\mathrm{b}$ fraction uncertainty from variations in the templates are found to be negligible.

The systematic errors and final value for the inclusive cross section are summarised in table 7.2. The final values together with systematic errors for each bin in the differential cross sections are shown in tables 7.3, 7.4 and 7.5 .

\subsection{Comparison to Previous Measurements}

Section 2.6 described how previous measurements for the b quark cross section showed discrepancies between data and Next to Leading Order theoretical predictions. The event selection in this analysis enhances the Leading Order contributions and the results show good agreement with Leading Order as well as Next to Leading predictions for these type of events. As a result this analysis is not directly comparable to the previous measurements that have been made. Both CDF and D0 are working on inclusive b jet cross section analyses [57] where only only one jet is required in the event selection and so both Leading Order and Next to Leading Order contributions are present. The inclusive b jet analyses are comparable with previous measurements. Since this analysis agrees with theoretical prediction a discrepancy for the inclusive $\mathrm{b}$ jet analyses would indicate that QCD calculations appear to be incorrect at Next to Leading Order. Results of B hadron production 


\begin{tabular}{|c|c|}
\hline Systematic Uncertainty & $\sigma_{b \bar{b}}(\mathrm{nb})$ \\
\hline$F_{b}$ & \pm 1.0 \\
\hline Luminosity & \pm 2.1 \\
\hline$\epsilon_{b}^{l e a d}$ & \pm 4.6 \\
\hline Templates Used & \pm 1.2 \\
\hline Correction Factor & \pm 2.7 \\
\hline$E_{t}$ Dependence & \pm 3.6 \\
\hline$\epsilon_{b}^{o t h e r}$ & \pm 3.9 \\
\hline Templates Used & \pm 1.2 \\
\hline Correction Factor & \pm 2.7 \\
\hline$E_{t}$ Dependence & \pm 2.5 \\
\hline Acceptance & $\begin{array}{l}+6.5 \\
-7.0\end{array}$ \\
\hline Relative Correction & $\begin{array}{l}+3.2 \\
-3.7\end{array}$ \\
\hline Energy Scale Correction & $\begin{array}{l}+4.4 \\
-4.7\end{array}$ \\
\hline Multiple Interaction Correction & \pm 0 \\
\hline Absolute Correction & $\begin{array}{l}+2.8 \\
-2.9\end{array}$ \\
\hline b Jet Correction & ${ }_{-1.3}^{+1.4}$ \\
\hline PDFs & \pm 1.6 \\
\hline Final Value & $34.5 \pm 1.8_{-9.5}^{+9.1}$ \\
\hline
\end{tabular}

Table 7.2: Systematic errors on the cross section and the final value with the associated statistical and systematic error. The contributions to the total systematic error are in bold. Each contribution is split into its various components. 


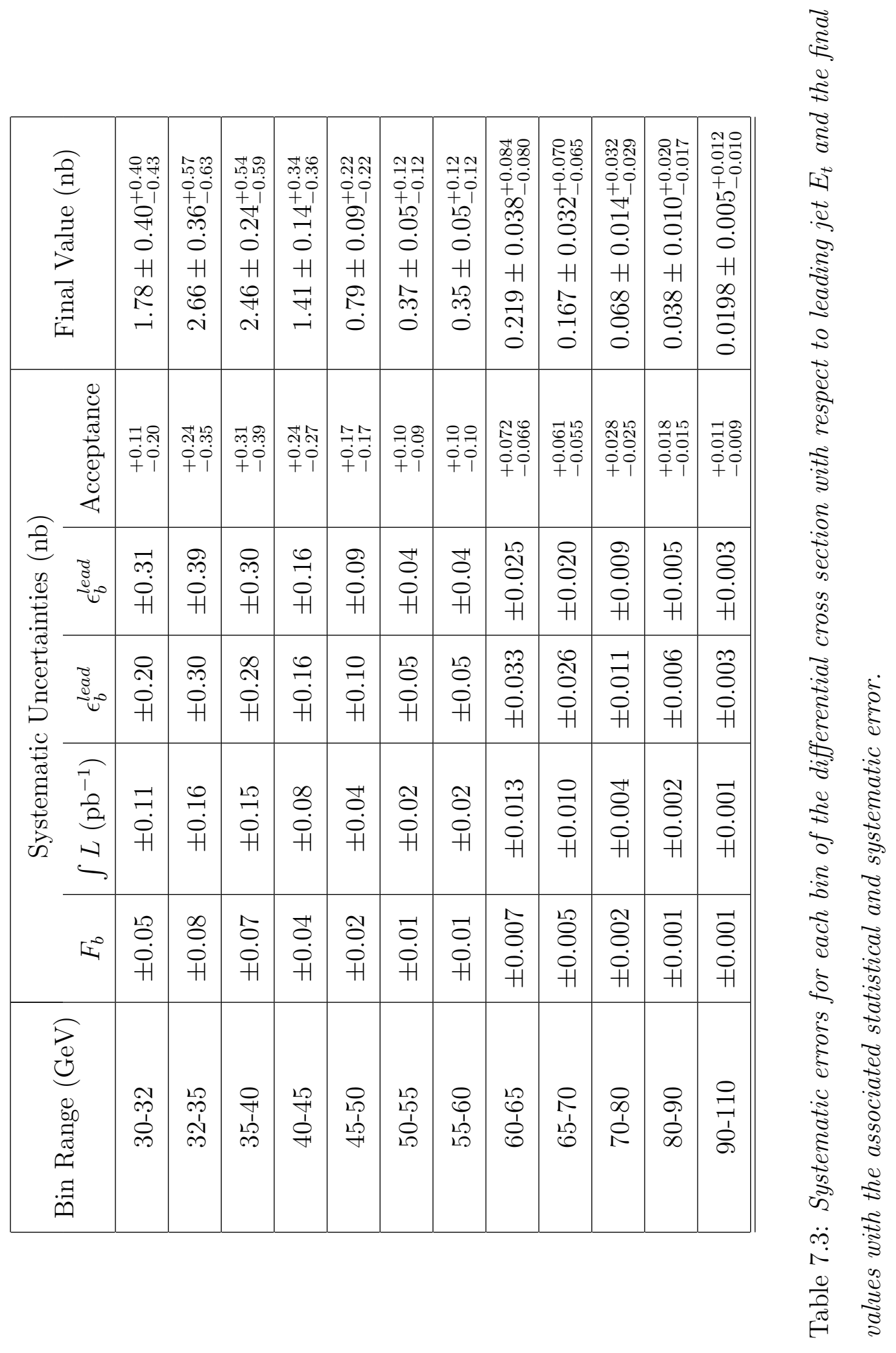




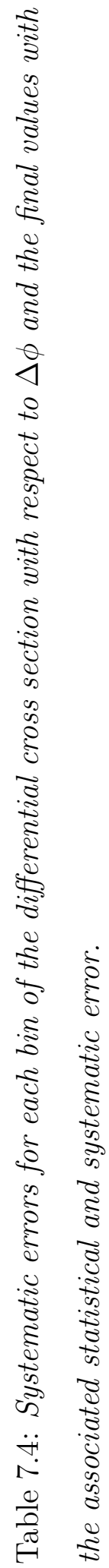




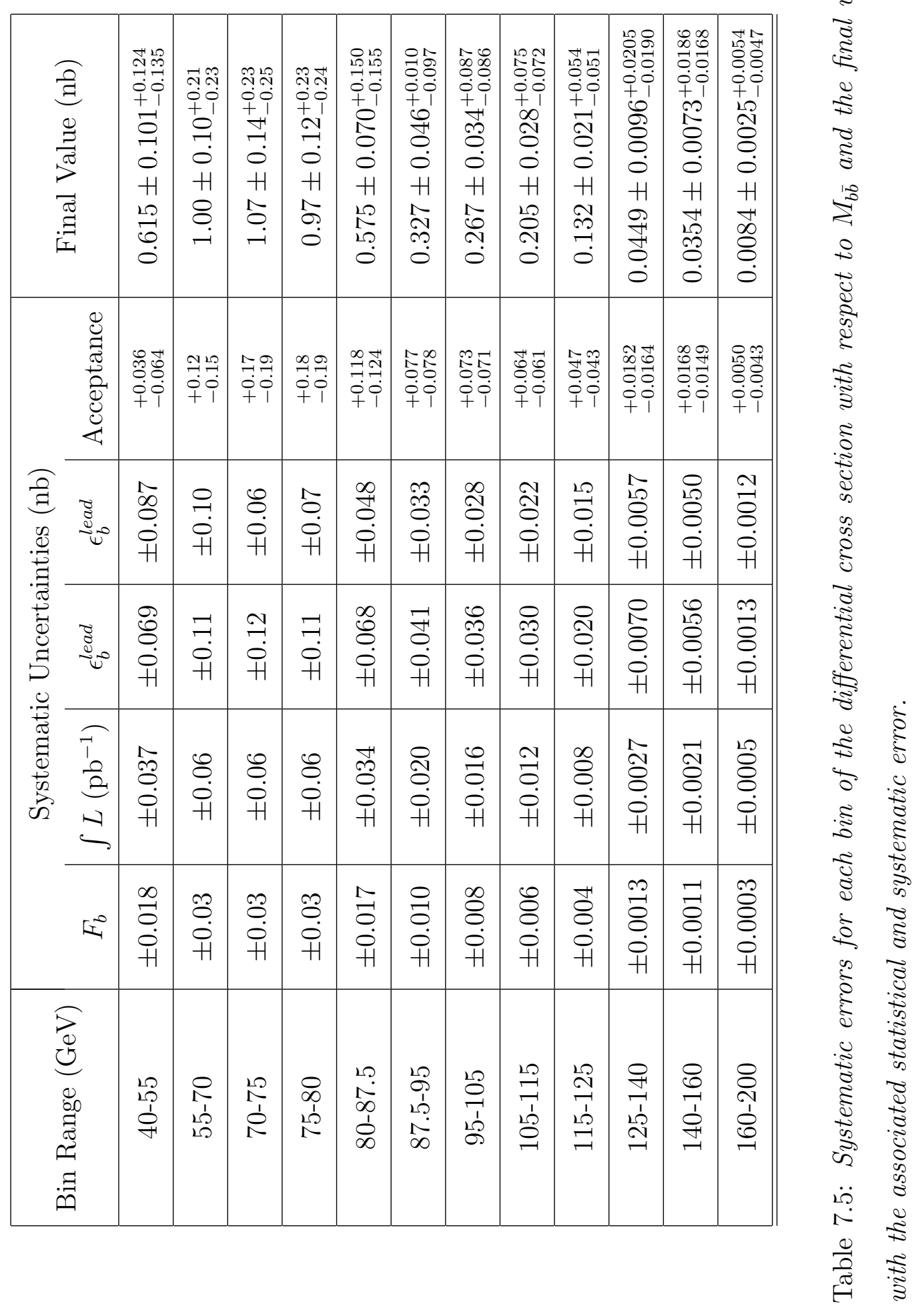


cross sections have also been presented by CDF and D0 at recent conferences [57], these have shown good agreement with Next to Leading Order theoretical predictions. 


\section{Chapter 8}

\section{Conclusions}

The inclusive $b \bar{b}$ jet production cross section in $p \bar{p}$ collisions at $\sqrt{s}=1.96 \mathrm{TeV}$ has been measured. For events containing two tagged jets within $|\eta|<1.2$, where one of the jets has $E_{t}^{c o r}>30 \mathrm{GeV}$ and the other $E_{t}^{c o r}>20 \mathrm{GeV}$ it is found to be $34.5 \pm 1.8_{-9.5}^{+9.1} \mathrm{nb}$ in data. This is in good agreement with the Pythia(CTEQ51) ${ }^{1}$ prediction of $38.7 \pm 0.6 \mathrm{nb}$ and the MC@NLO prediction of $35.7 \pm 2.0 \mathrm{nb}$, where JIMMY is used to describe the underlying event. Measurements of the differential cross section as a function of jet Et and dijet invariant mass also show good agreement between Pythia, MC@NLO and data.

The differential cross section as a function of azimuthal angle shows that the event selection picks out the Leading Order flavour creation processes. This explains why data are in such good agreement with Pythia predictions, which only includes the Leading Order diagrams and generates the flavour excitation and gluon splitting process through parton showers. At small opening angles the effect of Next to Leading Order corrections is apparent,

\footnotetext{
${ }^{1}$ The underlying event is tuned to CDF data.
} 
the measured cross section deviates from Pythia at these small opening angles. This suggests that although the overall cross section is correct in Pythia the production mix of the three types of processes may be slightly wrong. The differential cross section as a function of $\Delta \phi$ for MC@NLO shows better agreement with data than Pythia, suggesting that the Next to Leading Order contributions in MC@NLO are correct. Calculations by S. Frixione et al. [58] also show Next to Leading Order predictions are very similar to Leading Order when two central b hadrons are required within the event.

The investigations into the differences between Monte Carlo samples show the underlying event contributions have a large effect on the cross section measurement. When the default Herwig simulation is used for the underlying event, MC@NLO predictions for jets are found to be too low, despite agreement with Pythia at the b hadron level. 


\section{Appendix A}

\section{Equations Defining Tower}

\section{Kinematics}

$$
\begin{gathered}
E_{E M_{T}}=E_{E M} \sin \theta_{E M}, E_{H A D_{T}}=E_{H A D} \sin \theta_{H A D} \\
E_{T}=E_{E M_{T}}+E_{H A D_{T}} \\
\eta=\frac{\eta_{E M} E_{E M_{T}}+\eta_{H A D} E_{H A D_{T}}}{E_{T}} \\
\phi=\frac{\phi_{E M} E_{E M_{T}}+\phi_{H A D} E_{H A D_{T}}}{E_{T}} \\
p_{x}=E_{E M} \cos \phi_{E M} \sin \theta_{E M}+E_{H A D} \cos \phi_{H A D} \sin \theta_{H A D} \\
p_{y}=E_{E M} \sin \phi_{E M} \sin \theta_{E M}+E_{H A D} \sin \phi_{H A D} \sin \theta_{H A D} \\
p_{z}=E_{E M} \cos \theta_{E M}+E_{H A D} \cos \theta_{H A D}
\end{gathered}
$$

where:

- $E_{E M}$ is the electromagnetic energy in the tower.

- $E_{H A D}$ is the hadronic energy in the tower. 
- $\theta_{E M}$ is the polar angle from the vertex to the centre of the electromagnetic component of the tower.

- $\theta_{H A D}$ is the polar angle from the vertex to the centre of the hadronic component of the tower.

- $\phi_{E M}$ is the azimuthal angle of the tower measured in the electromagnetic component.

- $\phi_{H A D}$ is the azimuthal angle of the tower measured in the hadronic component. 


\section{Appendix B}

\section{Checking the 60:40 Energy}

\section{Ratio}

The Jet Clustering algorithm takes calorimeter towers as an input. To reconstruct jets using stable particles in HEPG they first need to be transformed into calorimeter towers. The energy of the particle is split 60:40 between the hadronic and electromagnetic components of the tower into which the particle would enter and the tower kinematics are then defined as in appendix A. How the kinematics are affected by the energy splitting ratio is described here.

For this example a tower located at an $\eta$ of 0.3 and $\phi$ of $0.2 \mathrm{rad}$ is used. The calorimeters are arranged such that they point to the centre of the detector, if the primary interaction vertex is at this point then $\theta_{E M}$ and thet $a_{H A D}$ are equal to one another. If this is shifted slightly then the $\theta_{E M}$ and thet $a_{H A D}$ are different, as shown in figure B.1.

A uniform distribution is used to generate a random number between 0 and 10, this is taken to represent the energy of the particle entering the tower 


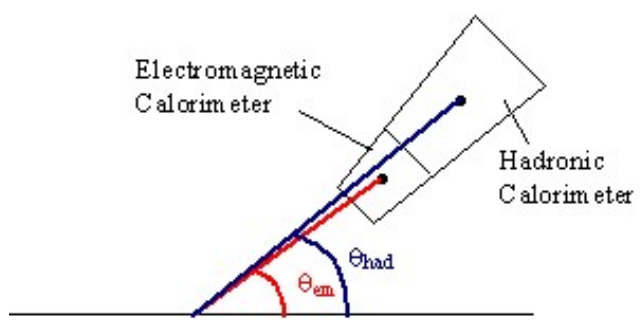

Figure B.1: Schematic diagram showing how theta $a_{E M}$ and theta $a_{H A D}$ are different if the interaction vertex is shifted from the centre.

in $\mathrm{GeV} .60 \%(40 \%)$ of the energy is assigned to the hadronic(electromagnetic) component and the tower kinematics are calculated for an interaction vertex at zero and one located at $20 \mathrm{~cm}$ away in the $\mathrm{z}$ direction. To check the energy assignment a second method is used to split the energy, the fraction to be assigned to the hadronic component is randomly chosen from a guassian distribution with a mean of 0.5 and a sigma of 0.15 . After splitting the tower kinematics are again calculated for a vertex at zero and $20 \mathrm{~cm}$. Figure B.2 shows the difference in the tower energy for the 60:40 splitting and the gaussian splitting for a vertex at zero and $20 \mathrm{~cm}$. If the vertex is at zero then there is no difference between the two methods, this is because $\theta_{E M}$ and thet $a_{H A D}$ are equal and so the electromagnetic and hadronic components are simply added together. For a vertex at $20 \mathrm{~cm}$ the effect of using the two different methods is still only very small. 


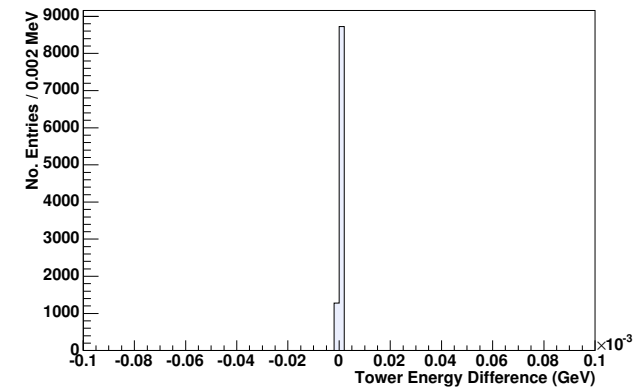

(a) Interaction Vertex at 0

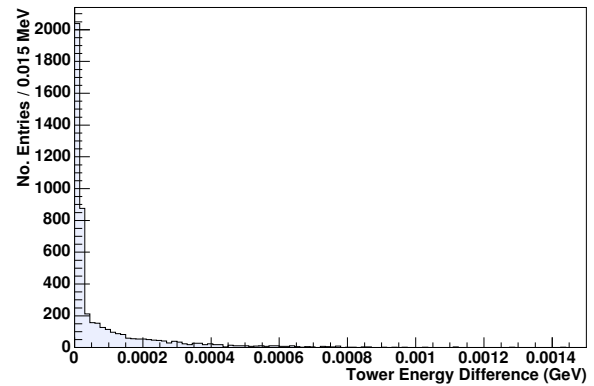

(b) Interaction Vertex at $20 \mathrm{~cm}$

Figure B.2: The difference between using at 60:40 ratio to split the energy for a particle and using a Gaussian distribution to split the energy for a) an interaction vertex located at 0 and b) an interaction vertex located $20 \mathrm{~cm}$ from 0 in the $z$ direction. 


\section{Appendix C}

\section{Plots for Jet Energy Ratio}

\section{using Herwig}

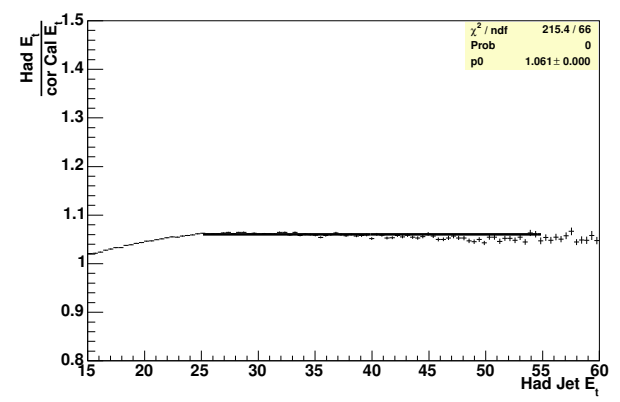

Figure C.1: Profile plot showing hadron (Had) jet $E_{t}$ divided by corrected calorimeter (cor Cal) jet $E_{t}$, as a function of the hadron jet $E_{t}$. The solid line represents a fit to a constant function, the fit parameters are shown in the top right corner. 


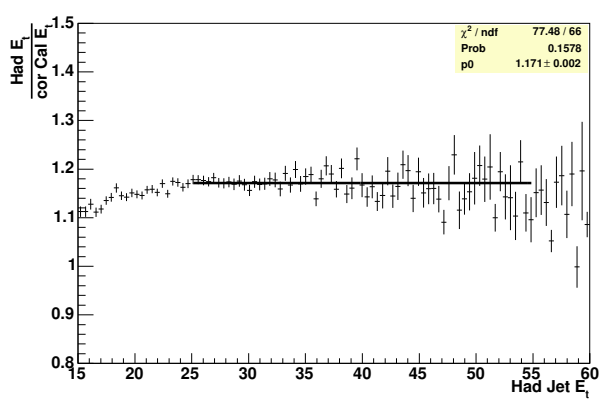

(a) b-jets

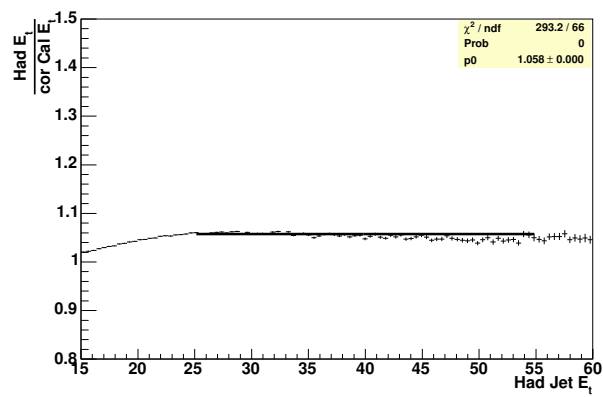

(c) gluon-jets

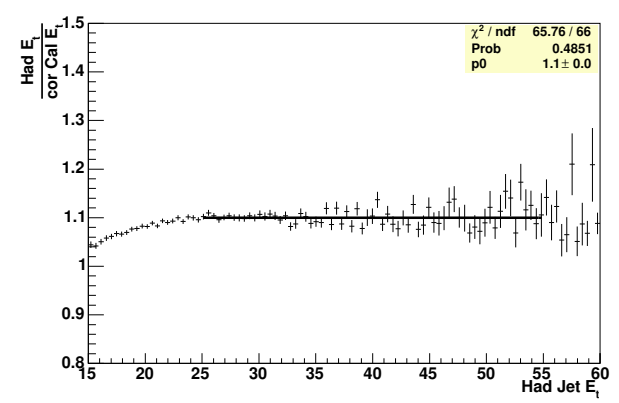

(b) c-jets

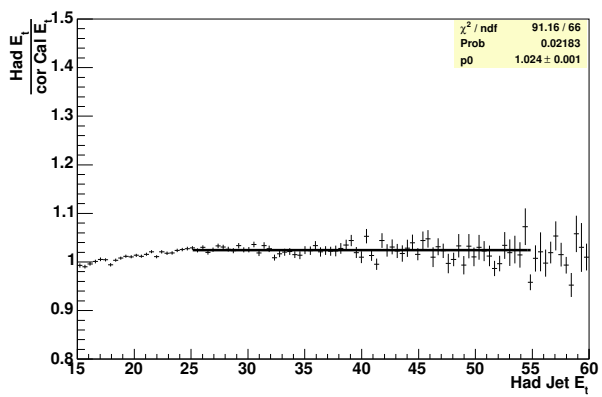

(d) uds-jets

Figure C.2: Profile plots showing hadron (Had) jet $E_{t}$ divided by corrected calorimeter (cor Cal) jet $E_{t}$, as a function of the hadron jet $E_{t}$, for: a) bjets; b) c-jets; c) gluon-jets; d) uds-jets. The solid line represents a fit to a constant function, the fit parameters are shown in the top right corner of each plot. 

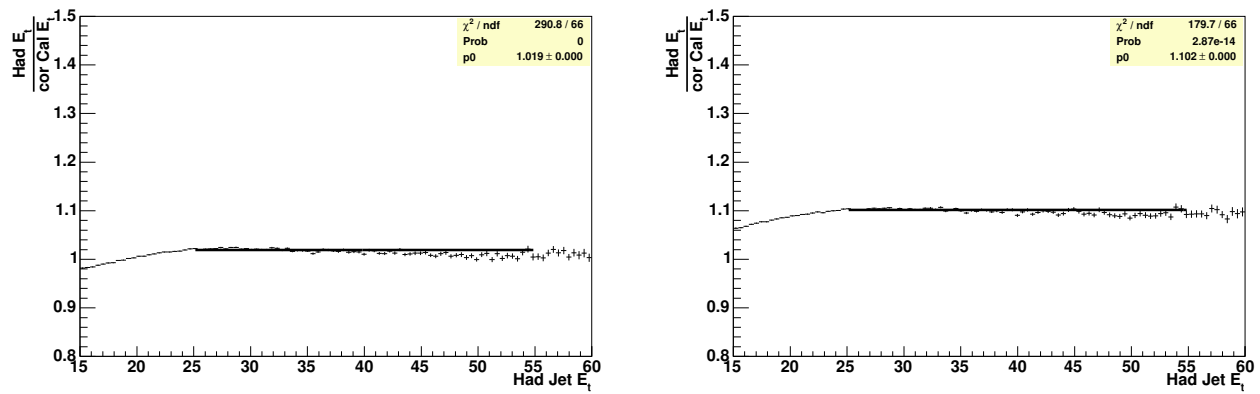

Figure C.3: Profile plots showing the ratio of hadron (Had) jet $E_{t}$ to corrected calorimeter (cor Cal) jet $E_{t}$, for gluon- and uds-jets combined, when the relative energy correction is adjusted by $+1 \sigma$ (left) and $-1 \sigma$ (right). The solid line represents a fit to a constant function, the fit parameters are shown in the top right corner.
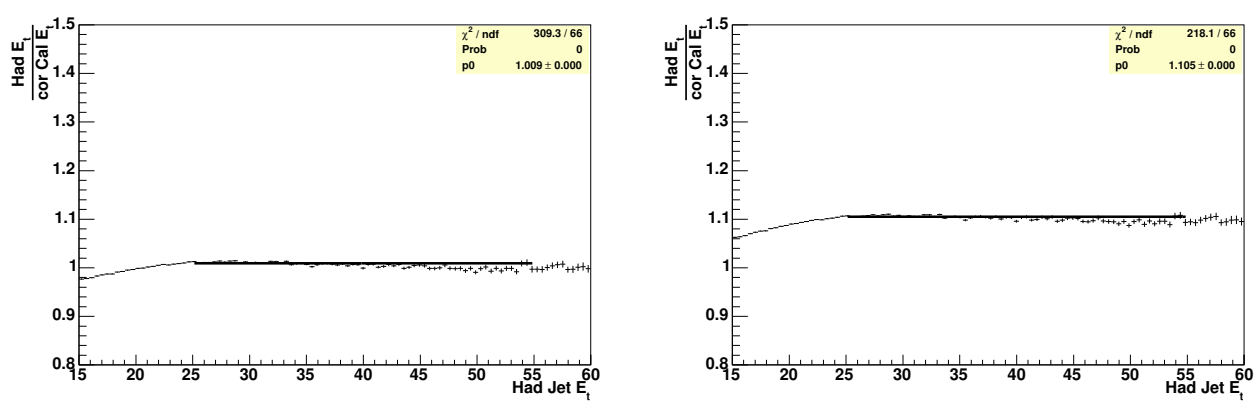

Figure C.4: Profile plots showing the ratio of hadron (Had) jet $E_{t}$ to corrected calorimeter (cor Cal) jet $E_{t}$, for gluon- and uds-jets combined, when the jet energy scale correction is adjusted by $+1 \sigma$ (left) and $-1 \sigma$ (right). The solid line represents a fit to a constant function, the fit parameters are shown in the top right corner. 

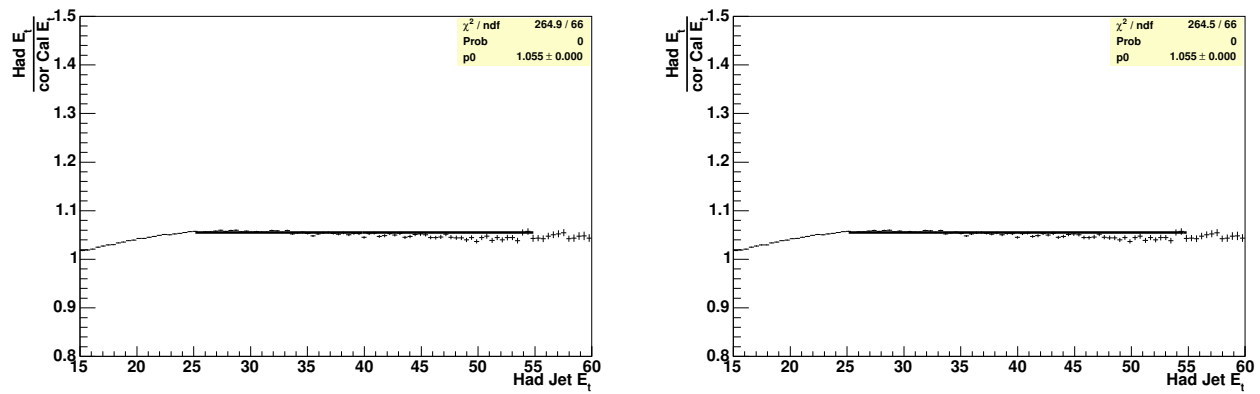

Figure C.5: Profile plots showing the ratio of hadron (Had) jet $E_{t}$ to corrected calorimeter (cor Cal) jet $E_{t}$, for gluon- and uds-jets combined, when the multiple interaction correction is adjusted by $+1 \sigma$ (left) and $-1 \sigma$ (right). The solid line represents a fit to a constant function, the fit parameters are shown in the top right corner.
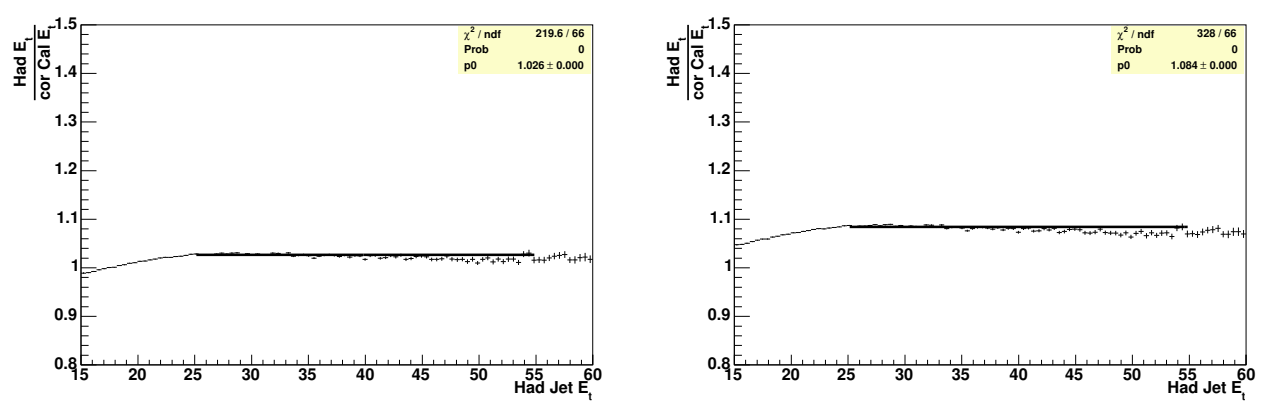

Figure C.6: Profile plots showing the ratio of hadron (Had) jet $E_{t}$ to corrected calorimeter (cor Cal) jet $E_{t}$, for gluon- and uds-jets combined, when the absolute energy correction is adjusted by $+1 \sigma$ (left) and $-1 \sigma$ (right). The solid line represents a fit to a constant function, the fit parameters are shown in the top right corner. 


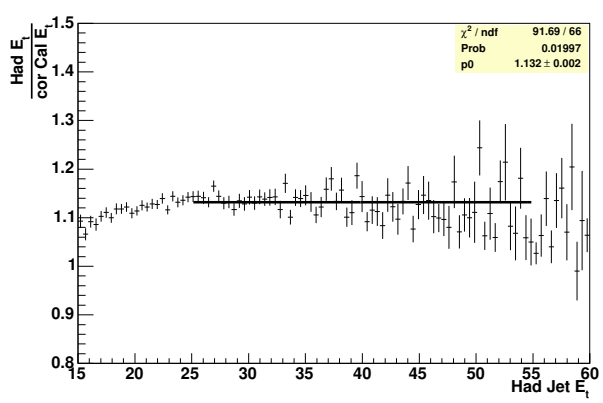

(a) non semi-leptonic decays

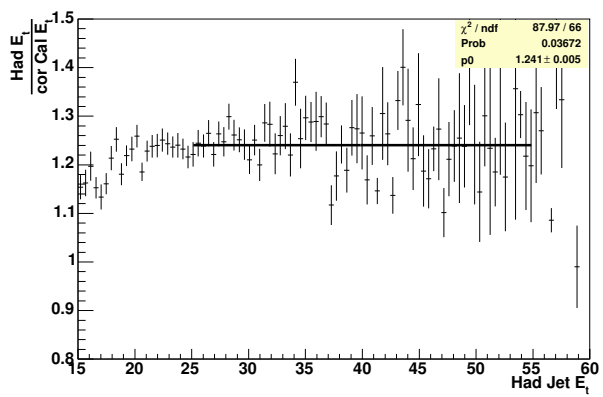

(c) muon in decay chain

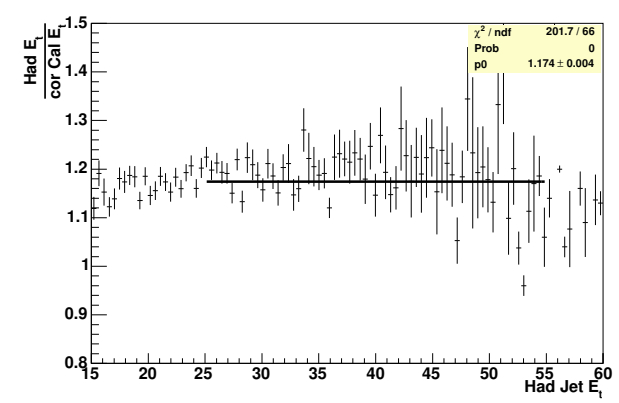

(b) electron in decay chain

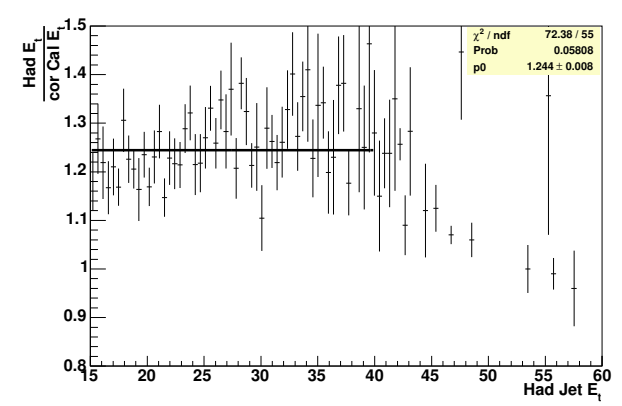

(d) tau in decay chain

Figure C.7: Profile plots showing hadron (Had) jet $E_{t}$ divided by corrected calorimeter (cor Cal) jet $E_{t}$, as a function of the hadron jet $E_{t}$, for: a) non semi-leptonic $b$ decay; b) b decay involving an electron; c) b decay involving $a$ muon; d) b decay involving a tau. The solid line represents a fit to a constant function, the fit parameters are shown in the top right corner. 


\section{Appendix D}

\section{Variables used for Electron}

\section{Identification}

$\boldsymbol{E}_{\boldsymbol{t}}: \quad$ The transverse energy deposited in the electromagnetic calorimeter for the cluster.

$\boldsymbol{p}_{\boldsymbol{t}}$ : The transverse momentum of the track linked to the electromagnetic cluster.

E/P: $\quad$ The ratio of $E_{t}$ to $p_{t}$.

Had/Em: The ratio of hadronic energy deposited to electromagnetic energy deposited by the cluster.

Lshr: The lateral shower profile for electrons. This variable compares the energy in CEM towers adjacent to the seed tower for data and test beam electrons [59].

CES $\boldsymbol{\Delta} \boldsymbol{x}: \quad$ The distance in the $\mathrm{r}-\phi$ plane between the extrapolated track and the shower maximum cluster. 
CES $\boldsymbol{\Delta} z: \quad$ The distance in the r-z plane between the extrapolated track and the shower maximum cluster.

CES strip $\chi^{2}$ : The $\chi^{2}$ comparison of the shower profile in the r-z view with the same profile extracted from test beam electrons. 


\section{Bibliography}

[1] P. W. Higgs, Broken Symmetries and the Masses of Gauge Bosons, Phys. Rev. Lett 13 (1964) 508.

[2] S. L. Glashow, Partial Symmetries of Weak Interactions, Nucl. Phys. 22 (1961) 579.

S. Weinberg, A Model of Leptons, Phys. Rev. Lett 19 (1967) 1264.

A. Salam, Elementary Particle Theory: Relativistic Groups on Analyticity, ed. N. Svartholm 367 (1969).

[3] J. C. Collins et al., Handbook of perturbative QCD, Rev. Mod. Phys. 67 (1995) 157.

[4] Proceedings of the School for Young High Energy Physicists held 1-13 September 2002, CCLRC Technical Report RAL-TR-2003-07 (2003).

[5] M. E. Peskin, D. V. Schroeder, An Introduction to Quantum Field Theory, Addison-Wesley Publishing (1995).

[6] M. Kobayashi and T. Maskawa, CP Violation in the Renormalizable Theory of Weak Interaction, Prog. Theor. Phys 49 (1973) 652.

[7] Sigurdur Helgason, Differential geometry, Lie groups, and symmetric spaces, Academic Press, New York (1978).

[8] Rick Field, The Sources of b-Quarks at the Tevatron and their Correlations, Phys. Rev. D65 (2002) 094006.

[9] T. Sjostrand et al., High-Energy-Physics Event Generation with PYTHIA 6.1, Comp. Phys. Comm. 135 (2001) 238.

[10] G. Corcella et al., HERWIG 6: An Event Generator for Hadron Emission Reactions With Interfering Gluons (Including Supersymmetric Processes), JHEP 01 (2001) 010. 
[11] S. Frixione et al., Matching NLO QCD Computations and Parton Shower Simulations, JHEP 06 (2002) 029.

[12] G. Altarelli, G. Parisi, Asymptotic Freedom in Parton Language, Nucl. Phys. B126 (1977) 298.

[13] H. L. Lai et al., Global QCD Analysis of Parton Structure of the Nucleon: CTEQ5 Parton Distributions, Eur. Phys. J. C 12 (2000) 375.

[14] A.D. Martin et al., Parton Distributions: a New Global Analysis, Eur. Phys. J. C4 (1998) 463.

[15] B. Andersson et al., Parton Fragmentation and String Dynamics, Phys. Rep. 97 (1983) 31.

[16] B. R. Webber, A QCD Model for Jet Fragmentation Including Soft Gluon Interference, Nucl. Phys. B238 (1984) 492.

[17] S. Eidelman et al., Review of Particle Physics, Phys. Lett. B592 1 (2004).

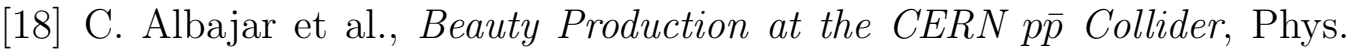
Lett B256 (1991) 121.

C. Albajar et al., Measurement of the Bottom Quark Production Cross Section in $p \bar{p}$ at $\sqrt{s}=0.63 T e V$, Phys. Lett. B213 (1988) 405.

[19] F. Abe et al., Inclusive J/ psi, psi (2S), and b-quark production in pbarp collisions at sqrt $s=1.8 \mathrm{TeV}$, Phys. Rev. Lett 69 (1992) 3704 .

F. Abe et al., Measurement of bottom quark production in $1.8 \mathrm{TeV} p \bar{p}$ collisions using muons from b-quark decays, Phys. Rev. Lett. 71 (1993) 2396.

F. Abe et al., Measurement of the B Meson Differential Cross Section $d \sigma / d p_{t}$ in $p \bar{p}$ collisions at $\sqrt{s}=1.8 T e V$, Phys. Rev. Lett. 75 (1995) 1451.

[20] S. Abachi et al., Inclusive and b-Quark Production Cross Sections in pp-bar Collisions at sqrt $s=1.8$ TeV, Phys. Rev. Lett. 74 (1995) 3548.

$\mathrm{S}$. Abachi et al. $J / \psi$, production in $p \bar{p}$ collisions at $\sqrt{s}=1.8 T e V$, Phys. Lett. B370 (1996) 239.

B. Abbott et al., The $b$ Production Cross Section and Angular Correlations in $p \bar{p}$ collisions at $\sqrt{s}=1.8 T e V$, Phys. Lett. B487 (2000) 264.

[21] C. Adloff et al., Measurement of open beauty production at HERA, Phys. Lett. B467 (1999) 156 , erratumibid. B518 (2001) 331.

[22] C. Peterson et al., Scaling violations in inclusive $e^{+} e^{-}$annihilation spectra, Phys. Rev. D27 (1983) 105. 
[23] M. Cacciari and P. Nason, Is There a Significant Excess in Bottom Hadroproduction at the Tevatron?, Phys. Rev. Lett. 89 (2002) 122003.

[24] M. Cacciari, M. Greco and P. Nason, The pt Spectrum in Heavy-Flavour Hadroproduction, JHEP 9805 (1998) 007.

[25] F. Abe et al., Observation of Top Quark Production in $p$ anti-p Collisions with the Collider Detector at Fermilab, Phys. Rev. Lett. 74 (1995) 2626 .

[26] S. van der Meer, Stochastic Cooling and the Accumulation of Antiprotons, Rev. Mod. Phys. 57 (1985) 689.

[27] The Run II Handbook, http://www-bd.fnal.gov/runII/index.html

[28] T. K. Nelson, The CDF-II Silicon Tracking System, Nucl. Instrum. Meth. A485 (2002) 1.

[29] A. Sill, CDF Run II Silicon Tracking Projects, Nucl. Instrum. Meth. A447 (2000) 1.

[30] S. Nahn, Status of the CDF Run II silicon detector, Nucl. Instrum. Meth. A511 (2003) 20.

[31] CDF Collaboration, The CDF II Detector: Technical Design Report, Fermilab-PUB-96/390-E (1996).

[32] T. Affolder et al., COT Central Outer Tracker, Nucl. Instrum. Meth. A526 (2004) 249.

[33] C. Grozis et al., A Time-Of-Flight Detector for CDF, Int. J.Mod. Phys. A16S1C (2001) 1119.

[34] L. Balka et al., The CDF Central Electromagnetic Calorimeter, Nucl. Instrum. Meth. A 267 (1988) 272.

[35] S. Bertolucci et al., The CDF Central and End wall Hadron Calorimeter , Nucl. Instrum. Meth. A 267 (1988) 301.

[36] D. Acosta et al., The CDF Luminosity Monitor, Nucl. Instrum. Meth. A 461 (2001) 540.

[37] D. Acosta et al., The Performance of the CDF Luminosity Monitor, Nucl. Instrum. Meth. A 494 (2002) 57. 
[38] Y. S. Chung et al., The Level-3 Trigger at the CDF Experiment at Tevatron Run II, yet to be published.

[39] J. Antos et al, Design and First Tests of the CDF Run II Farms, Comp. Phys. Comm 140 (2001) 239.

[40] G. C. Blazey et al., Run II Jet Physics, CDF Note 5293 (2000).

[41] J-F Arguin et al., Generic Jet Energy Corrections for Run II data used for the Winter Conferences, CDF note 6280 (2003).

[42] J-F Arguin et al., Systematic Uncertainties Associated with Jet Corrections for Winter 2003 Conferences, CDF note 6419 (2003).

[43] C. Hays et al., The COT Pattern Recognition Algorithm and Offline Code, CDF Note 6992 (2004).

[44] C. Chen et al., Measurement of the Track Reconstruction Efficiency in the Central Outer Tracking Using a Monte Carlo Track Embedding Technique, CDF Note 6394 (2003).

[45] W-M. Yao and K. Bloom, "Outside-In" Silicon Tracking at CDF, CDF Note 5991 (2002).

[46] W. Yao et al., A Seed Vertexing b-Tag Algorithm for Top, CDF Note 2716 (1994).

[47] J. Marriner, Secondary Vertex Fit with Mass and Pointing Constraints (CTVMFT), CDF NOTE 1996 (1993).

[48] ROOT TFractionFitter Page,

http://root.cern.ch/htmldoc/TFractionFitter.html

[49] R. Barlow and C. Beeston, Fitting Using Finite Monte Carlo Samples, Comp. Phys. Comm. 77 (1993) 219.

[50] Electron Task Force, http://www-cdf.fnal.gov/internal/physics/top/r2leptons/etf/etf_main.html

[51] Henri Bachacou et al., Efficiency of SECVTX B-Tag Used for 2003 Winter Conferences, CDF note 6291 (2003).

[52] A. Bhatti et al., First Measurement of the Inclusive Jet Cross Section in Run II, CDF note 6298 (2003). 
[53] S. Klimenko et al., Averaging of the inelastic cross sections measured by the CDF and the E811 experiments, FERMILAB-FN-0741 (2003).

[54] R. Field, The Underlying Event in Hard Scattering Processes, hepph/0201192 (2002).

[55] H. Plothow-Besch, PDFLIB - The Parton Density Functions Library, CERN-PPE W5051 (2000).

[56] JIMMY Generator - Multiparton Interactions in HERWIG, http://jetweb.hep.ucl.ac.uk/JIMMY/index.html

[57] M. D'Onofrio, Beauty production cross section measurements at Ecm = $1.96 \mathrm{TeV}$, hep-ex/0505036 (2005).

[58] S. Frixione et al., Matching NLO QCD and parton showers in heavy flavour production, J. High Energy Phys. JHEP08 (2003) 007.

[59] R. G. Wagner, Electron Identification for Run II: Understanding and Using Lshr, CDF Note 6249 (2003). 